\title{
HARISH-CHANDRA MODULES WITH THE UNIQUE EMBEDDING PROPERTY
}

BY

DAVID H. COLLINGWOOD

\begin{abstract}
Let $G$ be a connected semisimple real matrix group. In view of Casselman's subrepresentation theorem, every irreducible admissible representation of $G$ may be realized as a submodule of some principal series representation. We give a classification of representations with a unique embedding into principal series, in the case of regular infinitesimal character. Our basic philosophy is to link the theory of asymptotic behavior of matrix coefficients with the theory of coherent continuation of characters. This is accomplished by using the "Jacquet functor" and the Kazhdan-Lusztig conjectures.
\end{abstract}

Let $G$ be a connected semisimple real matrix group. In his subquotient theorem [7], Harish-Chandra established a fundamental connection between irreducible admissible representations and principal series representations. Casselman strengthened this connection, proving the subrepresentation theorem [2]: Every irreducible admissible representation may be realized as a submodule of some principal series representation. Further, Casselman's proof (which proceeds via a "reciprocity formula") suggests that, in general, several such embeddings exist. In this paper we address the following problem: Classify those irreducible admissible representations which have a unique embedding into a unique principal series representation (along a fixed minimal parabolic subgroup.) Our main result gives a classification of such representations in the case of regular infinitesimal character. Aside from historical interest, this problem has an important formulation in category $0^{\prime}$; cf. (1.24). Precisely, there exists an exact covariant functor $V \leadsto \hat{V}$ from the category of Harish-Chandra modules (cf. (1.6)) into category $\hat{O}^{\prime}$. (We will define $\hat{V}$ in a moment.) This functor has been studied by Casselman [2], Hecht-Schmid [11], Schmid [20] and Wallach [32]. A solution to our problem is equivalent to classifying admissible representations $V$, which have $\hat{V}$ an irreducible quotient of some Verma module. This shifts our problem into a new context, namely, investigating the structure of the modules $\hat{V}$. Many of our techniques, as well as some of our results, are couched in this more general setting.

We will state our main results precisely, referring to later sections for some of the definitions. Fix an Iwasawa decomposition $G=K A_{m} N_{m}$ and a minimal parabolic

Received by the editsis November 22, 1982.

1980 Mathematics Subject Classification. Primary 22E45; Secondary 20G05.

Key words and jiirases. Representations of semisimple Lie groups, embedding theorems, asymptotic behavior of matiix coefficients, coherent continuation, composition series problem, Kazhdan-Lusztig conjectures.

c/1984 American Mathematical Society $0002-9947 / 84 \$ 1.00+\$ .25$ per page 
subgroup $P_{m}=M_{m} A_{m} N_{m}$. A unique embedding property module $V$ (for $G$ ), abbreviated UEP-module, is defined to be an irreducible Harish-Chandra module with regular infinitesimal character and the property: there exists a unique (up to equivalence) irreducible $M_{m} A_{m}$-module $U$, such that $\operatorname{Hom}_{(\mathrm{g}, K)}\left(V, I_{P_{m}}^{G}(U)\right)=\mathrm{C}$; here, $I_{P_{m}}^{G}(U)$ is a (normalized) generalized principal series along $P_{m}$. Next, given any Harish-Chandra module $V$, let $V^{\prime}$ be the contragredient. Define $\hat{V}=\left(V^{\prime}\right)^{\left[\mathfrak{n}_{m}^{+}\right]}$, where $\mathfrak{p}_{m}=\mathfrak{m}_{m} \oplus \mathfrak{a}_{m} \oplus \mathfrak{n}_{m}^{-}$is the opposite minimal parabolic subalgebra and $(\cdots)^{\left[n_{m}^{-}\right]}$is defined as in $[\mathbf{2 0}, \S 4]$. We call $\hat{V}$ the asymptotic module of $V$. The first step in classifying UEP-modules is to show

(0.1) Proposition ((1.20), (1.25)). Let $V$ be an irreducible Harish-Chandra module with regular infinitesimal character. Then the following are equivalent: (a) $V$ is a UEP-module; (b) $H_{0}\left(\mathfrak{n}_{m}, V\right)$ is $M_{m} A_{m}$-irreducible; (c) $H_{0}\left(\mathfrak{n}_{m}, V\right)$ is $\mathfrak{m}_{m} \oplus \mathfrak{a}_{m^{-}}$ irreducible; (d) $\hat{V}$ is irreducible in category ${ }^{\circ}$.

The homology reformulation lends itself well to arguments involving "reduction to real rank one"; a general philosophy of this paper. Rephrasing the problem in category $\theta^{\prime}$ will be important in our use of the Kazhdan-Lusztig conjectures. Our main result is

(0.2) Theorem ((3.13), (3.38), (5.1)). (a) If $G$ is simple and rank $G \neq \operatorname{rank} K$, then the UEP-modules are precisely the finite-dimensional modules.

(b) If rank $G=\operatorname{rank} K$, then the UEP-modules are among the regular irreducible constituents of coherent continuations of the discrete series.

(c) If $G / K$ is Hermitian symmetric, then the UEP-modules are precisely the regular irreducible constituents of coherent continuations of the holomorphic discrete series.

Further refinements of $(0.2)(b)$ are subtle. For example,

(i) In order that $G$ possess non-finite-dimensional UEP-modules, it is necessary, but not sufficient, that $\operatorname{rank} G=\operatorname{rank} K$; cf. (6.1).

(ii) In order that $G$ possess non-finite-dimensional UEP-modules, it is sufficient, but not necessary, that $G / K$ be Hermitian symmetric; cf. Table 5 and (A.1.4).

(iii) The regular irreducible constituents of coherent continuations of a UEP-module need not be UEP-modules; cf. (3.35).

Finally, examination of the situation for $G=\mathrm{SP}(3,1)$ (cf. Table 5) indicates the complicated nature of our problem (in the case when $G / K$ carries no complex structure).

The contents of this paper can be described as follows. In $\$ 1$ we prove Proposition (0.1). This will hinge upon two "duality" results. First, $V$ is a UEP-module if and only if $V^{\prime}$ is a UEP-module. This relies upon an observation, due to D. Vogan [1], which insures we may "twist" $V$ into $V^{\prime}$ by an automorphism of $G$. (The linearity of $G$ is important here.) Secondly, we need the existence of a nondegenerate pairing $\left(V^{\prime}\right)^{\left[n_{m}^{-}\right]} \otimes(V)^{\left[n_{m}\right]} \rightarrow \mathbf{C}$, due to Hecht and Schmid [11]. We also point out the linearity of $G$ will be crucial in establishing the equivalence of $(0.1)(\mathrm{b}),(\mathrm{c})$.

In $\$ 2$, arguing by "reduction to real rank one", we show UEP-modules have integral infinitesimal character. Here, the key fact is that the property of having a 
unique embedding is hereditary in an appropriate sense. Also, results on the reducibility of generalized principal series for linear groups [23] will be used.

$\S \S 3-5$ constitute the heart of our paper. In $\S 3$ the first two parts of $(0.2)$ are established. Arguments for $(0.2)(a)$ are of a technical nature, depending upon the classification of real forms - but using $(0.1)$ in a crucial way. The proof of $(0.2)(b)$ is a consequence of the following observation: Whenever a UEP-module is tensored into the "worst growth chamber", we obtain a finite-dimensional constituent. This will amount to showing: Asymptotic modules of irreducible constituents of successive "wall crossings" of a UEP-module are completely reducible. It is here we employ the Kazhdan-Lusztig conjectures for Harish-Chandra modules and Verma modules, in the case of regular integral infinitesimal character. (In this setting, the Kazhdan-Lusztig conjectures are theorems.)

In $\$ 4$ Osborne's conjecture (for the minimal parabolic) and the aforementioned nondegenerate pairing lead us to a "character formula", linking characters of Harish-Chandra modules and formal characters of asymptotic modules; cf. [11]. In $\$ 5$ this "character formula" and Hecht's character formula for the coherent continuations of the holomorphic discrete series allow us to show: Regular irreducible constituents of coherent continuations of the holomorphic discrete series are UEPmodules. The remainder of $\$ 5$ is devoted to showing these modules exhaust all UEP-modules when $G / K$ is Hermitian symmetric. This exhaustion will follow from Langlands classification once we observe: For every UEP-module $V$ the $M_{m}$-structure of $H_{0}\left(n_{m}, V\right)$ is totally determined by the lowest weight of $H_{0}\left(n_{m}, V\right)$; cf. (3.36).

Finally, in $\S 6$ we consider the case of a split group, and $\S 7$ presents a technique applicable to any real rank one group-illustrated by $G=\operatorname{SP}(3,1)$.

Several people had previously obtained results on the UEP-problem. Casselman, Hecht, Schmid, Wallach and Zuckerman observed several years ago that holomorphic discrete series are UEP-modules. In her thesis, Silva [22] studied discrete series "embeddings" for real rank one groups, though her notion of "embedding" was in the sense of "subquotient". Wallach had observed (A.1.4) and computed all of the embeddings of the "analytic continuations of the holomorphic discrete series" [31]. Knapp and Wallach [13] obtained Szegö embeddings for the discrete series, while Schmid [21] gave discrete series embeddings into generalized principal series along maximal cuspidal parabolics.

ACKNOWLedgements. I am very grateful to Professor Henryk Hecht for suggesting the topic of this paper. He continuously gave generous advice, encouragement and understanding during the time he was my thesis advisor. I also wish to thank Professor Dragan Miličić for helpful suggestions on several points of this paper, as well as his constant interest in my progress. Finally, I want to thank Professor David Vogan for helpful discussions-most notably (3.39) and (7.5).

1. Reformulating the problem. For the most part, this section sets up notation and recalls certain connections between asymptotics, homology and intertwinings with generalized principal series. Our main result, Proposition (1.25), will reformulate the " unique embedding problem", using the language of $n_{m}$-homology and category $\theta^{\prime}$. 
Although connected semisimple real matrix groups will be the eventual objects of interest, it is technically advantageous to consider groups $G$ which satisfy the following axioms:

(1.1)(a) $G$ is a real reductive Lie group and $\operatorname{Ad}(G)$ is contained in the complex adjoint group of $\mathrm{g}$, where $\mathrm{g}$ denotes the complexification of the Lie algebra $\mathrm{g}_{0}$ of $G$.

(b) $G$ has finitely many connected components and the analytic subgroup of $G$ corresponding to $\left[\mathfrak{g}_{0}, \mathfrak{g}_{0}\right]$ has finite center.

(c) $G$ has a faithful finite-dimensional representation.

(d) Let $\mathfrak{h}_{0} \subseteq \mathfrak{g}_{0}$ be a Cartan subalgebra, and let $H$ be the centralizer of $\mathfrak{h}_{0}$ in $G$. Then $H$ is abelian.

Of course, (a) and (b) define the usual Harish-Chandra class of groups. Assumptions (c) and (d) are included chiefly to make (1.25) hold. This is the same class of groups studied in [30].

We now fix $G$ satisfying (1.1). It follows that there must exist a compact subgroup $K$ of $G$ which meets every component of $G$ such that $K \cap G^{0}$ is a maximal compact subgroup of $G^{0}=$ identity component of $G$. Observe that connected semisimple real matrix groups satisfy (1.1) and the standard properties of the former generalize to the latter. In particular, there exists a Cartan involution $\theta$ of $\mathfrak{g}_{0}$ associated to $\mathfrak{f}_{0}$, which lifts to an automorphism of $G$. (As a general rule, we denote real Lie algebras by Gothic lower case letters and subscript "0"; the complexifications without subscript.) Fix, once and for all, a compact subgroup $K$ and automorphism $\theta$ with the above described properties. Equip $\mathfrak{g}_{0}$ with a nondegenerate bilinear form $(1)$ $\operatorname{Ad}(G)$-invariant. Further, if $\mathfrak{g}_{0}=\mathfrak{f}_{0} \oplus \mathfrak{g}_{0}$ is the Cartan decomposition (relative to $\boldsymbol{\theta}$ ), we assume $(\mid)$ is positive definite on $\mathfrak{S}_{0}$ and negative definite on $\mathfrak{f}_{0}$. Restricting ( l) to any $\theta$-invariant subspace remains nondegenerate. We use the same notation to denote such a restriction, as well as the transported form on the complexified dual.

(1.2) The groups in (1.1) satisfy the crucial hereditary property: If $G$ is of type (1.1) and $A$ is a $\theta$-stable abelian subgroup, then the centralizer $G^{A}$ of $A$ in $G$ is again of type (1.1).

Let

$$
\mathfrak{h}_{m, 0}=\mathrm{t}_{m, 0} \oplus \mathfrak{a}_{m, 0} \quad\left(\text { resp. } H_{m}=T_{m} A_{m}\right)
$$

be a maximally split $\theta$-stable Cartan subalgebra of $\mathfrak{g}_{0}$ (resp. Cartan subgroup of $G$.) Here, $H_{m}$ is the centralizer of $\mathfrak{h}_{m, 0}$ in $G, T_{m}=H_{m} \cap K$ is compact, and $A_{m}$ is a vector group. We obtain root systems $\Phi\left(\mathfrak{g}, \mathfrak{h}_{m}\right)$ and $\Phi\left(\mathfrak{g}, \mathfrak{a}_{m}\right)$. Fix systems of positive roots $\Phi^{+}\left(\mathfrak{g}, \mathfrak{h}_{m}\right)$ and $\Phi^{+}\left(\mathfrak{g}, \mathfrak{a}_{m}\right)$ which satisfy the compatibility:

$$
\alpha \in \Phi\left(\mathfrak{g}, \mathfrak{h}_{m}\right),\left.\quad \alpha\right|_{\mathfrak{a}_{m}} \in \Phi^{+}\left(\mathfrak{g}, \mathfrak{a}_{m}\right) \Rightarrow \alpha \in \Phi^{+}\left(\mathfrak{g}, \mathfrak{h}_{m}\right) .
$$

It is useful to recall that the compatibility of (1.4) is equivalent to choosing positive systems which satisfy:

$$
\alpha \in \Phi^{+}\left(\mathfrak{g}, \mathfrak{h}_{m}\right),\left.\quad \alpha\right|_{a_{m}} \neq\left. 0 \Rightarrow \alpha\right|_{a_{m}} \in \Phi^{+}\left(\mathfrak{g}, \mathfrak{a}_{m}\right) .
$$

In all that follows, phrases such as, “. . the simple $\Phi^{+}\left(\mathfrak{g}, \mathfrak{h}_{m}\right)$ roots...", are meant to convey the fact we have chosen the simple roots determined by the fixed positive system $\Phi^{+}\left(\mathfrak{g}, \mathfrak{h}_{m}\right)$. 
Let $\mathfrak{g}_{0}=\mathfrak{f}_{0} \oplus \mathfrak{a}_{m, 0} \oplus \mathfrak{n}_{m, 0}$ and $G=K A_{m} N_{m}$ denote the Iwasawa decompositions determined by (1.4); $\mathfrak{p}_{m, 0}=\mathrm{m}_{m, 0} \oplus \mathfrak{a}_{m, 0} \oplus \mathfrak{n}_{m, 0}$ and $P_{m}=M_{m} A_{m} N_{m}$ the Langlands decompositions of the corresponding minimal parabolic subalgebra and subgroup. Having specified $\Phi^{+}\left(\mathfrak{g}, \mathfrak{h}_{m}\right)$ is equivalent to fixing a Borel subalgebra $\mathfrak{b}_{m}=\mathfrak{h}_{m} \oplus \mathfrak{r}$ with

$$
r=\left(m_{m} \cap r\right) \oplus n_{m} \quad \text { (semidirect product). }
$$

Notice that $\mathfrak{h}_{m} \oplus\left[\left(\mathfrak{m}_{m} \oplus \mathfrak{a}_{m}\right) \cap \mathfrak{r}\right]$ is a Borel subalgebra of the Levi factor $\mathfrak{m}_{m} \oplus \mathfrak{a}_{m}$; hence it determines a (compatible) positive root system $\Phi^{+}\left(m_{m} \oplus a_{m}, \mathfrak{h}_{m}\right)$ for

$$
\Phi\left(m_{m} \oplus \mathfrak{a}_{m}, \mathfrak{h}_{m}\right)=\left\{\alpha \in \Phi\left(\mathfrak{g}, \mathfrak{h}_{m}\right):\left.\alpha\right|_{a_{m}}=0\right\} .
$$

In all that follows, assume $P_{m}=M_{m} A_{m} N_{m}$ has been fixed along with the data required to define it.

By a Harish-Chandra module for $G$ we shall mean a module $V$ over the universal enveloping algebra $\mathfrak{U}(\mathfrak{g})$, together with an action of $K$, such that:

(1.6)(a) The representation of $K$ is an algebraic direct sum of irreducible finitedimensional continuous representations, each isomorphism class occurring with finite multiplicity.

(b) The representation of $\mathfrak{f}$ as a subalgebra of $g$ coincides with the differential of the $K$-action.

(c) For any $X \in \mathfrak{U}(\mathfrak{g}), k \in K$ and $v \in V$,

$$
\text { Ad } k(X) \cdot v=k \cdot X \cdot k^{-1} \cdot v \text {. }
$$

(d) $V$ has finite length as a $\mathfrak{U}(\mathfrak{g})$-module.

Recall, the space of $K$-finite vectors in an admissible finite length representation of $G$ on a Banach space is the standard example of a Harish-Chandra module. If $V$ is a Harish-Chandra module for $G$, then $V^{*}$ (the full algebraic dual of $V$ ) admits a natural $K$-module and $\mathfrak{U}(g)$-module structure. Let $V^{\prime}$ denote the $K$-finite vectors of $V^{*}$. We call $V^{\prime}$ the contragredient of $V$, which is itself a Harish-Chandra module.

Utilize $\Phi^{+}\left(g, a_{m}\right)$ to partially order the dual space $a_{m}^{*}$ :

(1.7) $\mu>\nu \Leftrightarrow \mu-\nu$ is a nonzero linear combination, with positive integral coefficients, of roots in $\Phi^{+}\left(g, a_{m}\right)$.

We also set $A_{m}^{-}=\exp \left(\mathfrak{a}_{m, 0}\right)^{-}$, where

$$
\begin{gathered}
\left(a_{m, 0}\right)^{-}=\left\{X \in a_{m, 0}:\langle\alpha, X\rangle<0 \text { for all } \alpha \in \Phi^{+}\left(\mathfrak{g}, a_{m}\right)\right\} . \\
\rho=\frac{1}{2} \sum_{\alpha \in \Phi^{+}\left(\mathfrak{g}, \mathfrak{h}_{m}\right)} \alpha, \quad \rho\left(P_{m}\right)=\frac{1}{2} \sum_{\alpha \notin \Phi^{+}\left(\mathfrak{g}, \mathfrak{a}_{m}\right)} n_{\alpha} \alpha,
\end{gathered}
$$

where $n_{\alpha}=$ dimension of the $\mathfrak{g}_{\alpha}$-root space.

Let $V$ be a Harish-Chandra module for $G$, which we may assume-by a result of Casselman-Prischepionok [2]-lifts to a global representation $\pi$ of $G$ : there exists a continuous representation $\pi$ on a Hilbert space $V_{\pi}$ such that $V$ may be identified, as a $(g, K)$-module, with the $K$-finite vectors of $V_{\pi}$. We call $V_{\pi}$ a globalization of $V$. Note that $V^{\prime} \subseteq\left(V_{\pi}\right)^{*}$ is the $K$-finite part. If $v \in V, v^{\prime} \in V^{\prime}$ we form a typical (real analytic) $K$-finite matrix coefficient

$$
f_{v, v^{\prime}}(x)=\left\langle\pi(x) v, v^{\prime}\right\rangle, \quad x \in G .
$$


The main result about asymptotic expansions of matrix coefficients on $A_{m}^{-}$, which is due to Harish-Chandra, has been summarized in our next theorem [3].

(1.9) ThEOREM. There exists a collection of polynomial functions $P_{v \cdot v^{\prime}}$ on $a_{m}$, indexed by $v \in V, v^{\prime} \in V^{\prime}$ and by $\nu$, which ranges over a countable set $\mathcal{E}(V) \subseteq a_{m}^{*}$ such that

$$
f_{v, v^{\prime}}(\exp X)=\sum_{\nu \in \mathcal{E}(v)} P_{v, v^{\prime}}^{\nu}(X) e^{\left\langle\nu+\rho\left(P_{m}\right), X\right\rangle}
$$

for $X \in\left(a_{m, 0}\right)^{-}$. This series converges uniformly and absolutely on any translate of $\left(a_{m, 0}\right)^{-}$whose closure is contained in $\left(a_{m_{0}}\right)^{-}$. A finite subset $\mathcal{E}_{l}(V) \subseteq \mathcal{E}(V)$ has the property that every $\nu \in \mathcal{E}(V)$ lies above some $\nu_{l} \in \mathcal{E}_{l}(V)$, relative to the ordering $<$.

Though formally unnecessary for our arguments, we have included (1.9) for completeness of exposition.

Let $P=M A N$ (resp. $\mathfrak{p}=\mathfrak{m} \oplus \mathfrak{a} \oplus \mathfrak{n}$ ) be the Langlands decomposition of a parabolic subgroup (resp. subalgebra); $\Phi(\mathfrak{g}, \mathfrak{a})=$ the roots of $a$ in $\mathfrak{g}$. Let $V$ be a $\mathfrak{U}(\mathrm{g})$-module. The functor $V \leadsto V / \mathfrak{n} V$ (resp. $V \leadsto \mathfrak{n}$-invariants of $V$ ) is covariant right exact (resp. covariant left exact). If we form the left (resp. right) derived functors, then we obtain the n-homology groups $H_{p}(\mathrm{n}, V)$ (resp. n-cohomology groups $\left.H^{p}(\mathrm{n}, V)\right)$ and $H_{0}(\mathrm{n}, V)=V / \mathrm{n} V\left(\right.$ resp. $H^{0}(\mathrm{n}, V)=$ space of $\mathrm{n}$-invariants of $\left.V\right)$. Since $\mathfrak{U}(\mathfrak{a}) \subseteq \mathfrak{Z}(\mathfrak{m} \oplus \mathfrak{a})=$ center of $\mathfrak{U}(\mathfrak{m} \oplus \mathfrak{a})$, we may decompose $\mathfrak{n}$-homology and $\mathfrak{n}$-cohomology into generalized $\mathfrak{a}$-weight spaces. Specifically,

$$
\begin{aligned}
& H_{p}(\mathfrak{n}, V)=\bigoplus_{\mu \in \mathfrak{a}^{*}} H_{p}(\mathfrak{n}, V)_{\mu}, \text { where } \\
& H_{p}(\mathfrak{n}, V)_{\mu}=\text { largest subspace of } H_{p}(\mathfrak{n}, V) \text { on which } \\
& X-\langle\mu, X\rangle \text { acts nilpotently, for all } X \in \mathfrak{a} ;
\end{aligned}
$$

A similar definition holds for cohomology.

Henceforth, unless otherwise indicated, we will assume

(1.11) $P$ is in standard position relative to $P_{m}$; i.e. $M_{m} \subseteq M, A \subseteq A_{m}$ and $N \subseteq N_{m}$.

We may identify $\Phi(\mathfrak{g}, \mathfrak{a})$ with a subset of $\Phi\left(\mathfrak{g}, \mathfrak{a}_{m}\right)$. Let

$$
\Phi\left(\mathrm{m} \oplus \mathfrak{a}, \mathfrak{h}_{m}\right)=\left\{\alpha \in \Phi\left(\mathfrak{g}, \mathfrak{h}_{m}\right):\left.\alpha\right|_{\mathfrak{a}}=0\right\} .
$$

The compatible choices (1.4) of $\Phi^{+}\left(\mathfrak{g}, \mathfrak{h}_{m}\right), \Phi^{+}\left(\mathfrak{m}_{m} \oplus \mathfrak{a}_{m}, \mathfrak{h}_{m}\right)$ and $\Phi^{+}\left(\mathfrak{g}, \mathfrak{a}_{m}\right)$ determine positive systems $\Phi^{+}\left(\mathfrak{m} \oplus \mathfrak{a}, \mathfrak{h}_{m}\right)$ and $\Phi^{+}(g, a)$ which satisfy the compatibility analogues of (1.4). We let $W\left(\mathfrak{g}, \mathfrak{h}_{m}\right)=$ Weyl group of $\Phi\left(\mathfrak{g}, \mathfrak{h}_{m}\right)$, similarly defining $W(\mathfrak{g}, \mathfrak{a}), W\left(\mathfrak{m} \oplus \mathfrak{a}, \mathfrak{h}_{m}\right)$, etc. Analogously to (1.8) define

$$
\begin{aligned}
& \rho_{\mathfrak{m} \oplus \mathfrak{n}}=\frac{1}{2} \sum_{\alpha \in \Phi^{+}\left(\mathfrak{m} \oplus \mathfrak{a}, \mathfrak{h}_{m}\right)} \alpha, \quad \rho_{\mathfrak{g}}=\rho, \\
& \rho(P)=\frac{1}{2} \sum_{\alpha \in \Phi(\mathfrak{g}, a)} n_{\alpha} \alpha,
\end{aligned}
$$

where $n_{\alpha}=$ dimension of the $\mathfrak{g}_{\alpha}$-root space.

Let $U$ be an irreducible Harish-Chandra module for $M A$. As previously remarked, $U$ globalizes to a representation $U_{\sigma}$ on $M A$. Extend $U_{\sigma}$ to $P=M A N$ by letting it act 
trivially on $N$. By right translation $G$ acts on the space of smooth functions $f$ : $G \rightarrow U_{\sigma}$ satisfying:

$$
f(p g)=U_{\sigma}(p) e^{\rho(P)}(p) f(g), \quad \text { for } g \in G, p \in P .
$$

If $I_{P}^{G}\left(U_{\sigma}\right)$ denotes the Hilbert space completion with respect to

$$
\left(f_{1} \mid f_{2}\right)=\int_{K}\left(f_{1}(k) \mid f_{2}(k)\right) d k
$$

then it is well known that $I_{P}^{G}\left(U_{\sigma}\right)$ is an admissible representation of $G$ of finite length. This yields a Harish-Chandra module for $G$ :

$$
I_{P}^{G}(U)=K \text {-finite vectors in } I_{P}^{G}\left(U_{\sigma}\right) .
$$

We will call this a generalized principal series representation (along $P$ ).

At this point we briefly recall the Harish-Chandra parameterization of infinitesimal characters: Let $P=M A N$ be a parabolic subgroup and $\mathfrak{p}=\mathrm{m} \oplus \mathfrak{a} \oplus \mathrm{n}$ its Lie algebra. Using the Harish-Chandra isomorphism, every algebra homomorphism $\chi$ : $3(m \oplus a) \rightarrow \mathbf{C}$, such that $\chi(1)=1$, is of the form $\chi_{\mathfrak{m} \oplus a, \gamma}: 3(m \oplus a) \rightarrow \mathbf{C}$, for some $\gamma \in \mathfrak{h}_{m}^{*}$. For our purposes a precise definition of $\chi_{\mathfrak{m} \oplus \mathfrak{a}, \gamma}$ is not necessary; see $[10,(3.21)]$. It is important to recall:

$$
\chi_{\mathfrak{m} \oplus a, \gamma}=\chi_{m \oplus a, \gamma^{\prime}} \Leftrightarrow \gamma \text { and } \gamma^{\prime} \text { lie in the same } W\left(\mathfrak{m} \oplus a, \mathfrak{h}_{m}\right) \text {-orbit. }
$$

Every irreducible Harish-Chandra module $U$ for $M A$ has an infinitesimal character $\chi_{\mathfrak{m} \oplus a, \gamma}$ for some $\gamma \in \mathfrak{h}_{m}^{*}$. We say $\chi_{\mathfrak{m} \oplus a, \gamma}$ is a regular infinitesimal character whenever $\gamma$ is regular in $\mathfrak{h}_{m}^{*}$; i.e. $(\gamma \mid \alpha) \neq 0$ for all $\alpha \in \Phi\left(\mathfrak{m} \oplus \mathfrak{a}, \mathfrak{h}_{m}\right)$.

Suppose $V$ is a Harish-Chandra module for $G$. Then $H_{*}(n, V)$ is a Harish-Chandra module for $M A[10,(2.24)]$. In case $P=P_{m}, H_{*}\left(\mathrm{n}_{m}, V\right)$ is a finite-dimensional $M_{m} A_{m}$-module.

(1.14) Theorem (Frobenius Reciprocity [2]). Let $U$ and $V$ be Harish-Chandra modules for $M A$ and $G$, respectively, with $U$ irreducible. Then

$$
\operatorname{Hom}_{(\mathrm{g}, K)}\left(V, I_{P}^{G}(U)\right)=\operatorname{Hom}_{(\mathrm{m} \oplus \mathrm{a}, K \cap M)}\left(H_{0}(\mathrm{n}, V), U \otimes e^{\rho(P)}\right) .
$$

(1.16) Remark. In case $P=P_{m}$, we actually have $\operatorname{Hom}_{M_{m} A_{m}}(\ldots, \ldots)$ on the right-hand side of (1.15).

Using the existence of asymptotic expansions (1.9), Casselman observed that $H_{0}\left(\mathrm{n}_{m}, V\right) \neq 0$. This fact, in conjunction with the evident isomorphism

$$
H_{0}\left(\mathrm{n}_{m}, V\right) \cong H_{0}\left(\mathrm{n}_{m} \cap \mathrm{m}, H_{0}(\mathrm{n}, V)\right)
$$

and Frobenius reciprocity, yields

(1.18) Corollary. Let $V$ be an irreducible Harish-Chandra module for $G$ and $P=M A N$ a parabolic subgroup of $G$. Then $V$ embeds as a submodule of some $I_{P}^{G}(U)$.

(1.19) REMARK. In particular, Casselman's subrepresentation theorem is a special case of (1.18) - the result to which we alluded in the Introduction.

A first reformulation of the "unique embedding problem" may now be carried out. Recall the definition of a UEP-module as given in the Introduction. (The definition given there makes perfectly good sense for $G$ of the type in (1.1).) 
(1.20) Lemma. Let $V$ be an irreducible Harish-Chandra module for $G$ with regular infinitesimal character. Then the following are equivalent:

(i) $V$ is a UEP-module.

(ii) $H_{0}\left(\mathfrak{n}_{m}, V\right)$ is an irreducible $M_{m} A_{m}$-module.

(iii) $H_{0}\left(\mathfrak{n}_{m}, V\right)$ is an irreducible $\mathrm{m}_{m} \oplus \mathfrak{a}_{m}$-module.

Proof. According to (1.14): the embeddings of $V$ into generalized principal series along $P_{m}$ are all determined by quotients of $H_{0}\left(\mathfrak{n}_{m}, V\right)$. Also [10,(6.6)], $H_{0}\left(\mathfrak{n}_{m}, V\right)$ is a semisimple $M_{m} A_{m}$-module. The equivalence of (i) and (ii) now follows, while (iii) $\Rightarrow$ (ii) is clear. Using (1.2), the Levi factor $M_{m} A_{m}$ of $P_{m}$ is again of the type in (1.1). By [30, 0.4.7], (ii) $\Rightarrow$ (iii). Q.E.D.

(1.21) REMARK. The necessity of some sort of infinitesimal regularity in (1.20) is easily seen by considering $G=\operatorname{SL}(2, \mathbf{R})$ and $V=$ the irreducible principal series with $a_{m}$ parameter $\nu=0$. Then $V$ has a unique embedding into principal series, yet $H_{0}\left(n_{m}, V\right)$ is not irreducible.

Let $V$ be a Harish-Chandra module for $G$. Form the opposite minimal parabolic $\theta\left(\mathfrak{p}_{m}\right)=\mathfrak{p}_{m}^{-}=\mathfrak{m}_{m} \oplus \mathfrak{a}_{m} \oplus \mathfrak{n}_{m}^{-}$. We define the asymptotic module of $V$ to be

$$
\hat{V}=\left\{v \in\left(V^{\prime}\right)^{*}:\left(\mathrm{n}_{m}^{-}\right)^{k} \cdot v=0, \text { some } k \in \mathbf{N}\right\} .
$$

In the notation of $[20, \S 4]$, our definition becomes $\hat{V}=\left(V^{\prime}\right)^{\left[\mathfrak{n}_{m}^{-}\right]}$. As defined here, $\hat{V}$ is a purely algebraic object. However, Hecht and Schmid have shown that $\hat{V}$ may be constructed solely in terms of the asymptotic expansions in (1.9)-hence the terminology "asymptotic module"; cf. [11].

(1.23) TheOrem (CASSElman-Hecht-Schmid). The association $V \leadsto \hat{V}$ determines an exact covariant functor from the category of Harish-Chandra modules for $G$ to the category of finitely generated $\mathfrak{U}(\mathfrak{g})$-modules which are $\mathfrak{p}_{m}^{-}$-finite and have finite $\mathfrak{a}_{m}$-multiplicities. For each Harish-Chandra module $V$, we have $\mathfrak{m}_{m} \oplus \mathfrak{a}_{m}$-isomorphisms

$$
H_{p}\left(\mathfrak{n}_{m}, V\right) \cong H_{p}\left(\mathfrak{n}_{m}, \hat{V}\right) \text { for all } p
$$

(1.24) Remarks. (i) All but the last statement is due to Casselman [20]. The homology assertion is due to Hecht-Schmid [11].

(ii) Denote by $\mathfrak{b}_{m}^{-}=\mathfrak{h}_{m} \oplus \mathfrak{r}^{-}$the Borel subalgebra opposite $\mathfrak{b}_{m}((1.5))$.

Henceforth, unless otherwise indicated, Verma modules are constructed relative to $\mathfrak{b}_{m}^{-}$.

Consequently, in view of (1.4), $M(\gamma)=\mathfrak{H}(\mathfrak{g}) \otimes_{\mathfrak{u}_{\left(\mathfrak{b}_{m}^{-}\right)}} \mathbf{C}_{\gamma+\rho}$ is the Verma module of lowest weight $\gamma+\rho, L(\gamma)=$ the unique irreducible quotient of $M(\gamma)$.

Category $\mathcal{O}^{\prime}$ consists of all finitely generated $\mathfrak{U}(\mathfrak{g})$-modules which are $\mathfrak{b}_{m}^{-}$-finite. Recall:

Every $W \in \theta^{\prime}$ has a finite composition series, the irreducible subquotients of which are irreducible quotients of Verma modules.

If $V$ is a Harish-Chandra module, then (1.23) shows $\hat{V} \in \Theta^{\prime}$. 
(1.25) Proposition. Let $G$ be a connected semisimple real matrix group and $V$ an irreducible Harish-Chandra module for $G$ with regular infinitesimal character. The following are equivalent:

(i) $V$ is a UEP-module.

(ii) $\hat{V}$ is irreducible in category $\theta^{\prime}$.

Proof. Suppose $\hat{V}$ is irreducible in $\mathcal{O}^{\prime}$. By (1.5) and (1.23), we have $\mathfrak{h}_{m}$-isomorphisms

$$
H_{0}(\mathrm{r}, \hat{V}) \cong H_{0}\left(\mathrm{r} \cap \mathrm{m}_{m}, H_{0}\left(\mathrm{n}_{m}, \hat{V}\right)\right) \cong H_{0}\left(\mathrm{r} \cap \mathrm{m}_{m}, H_{0}\left(\mathrm{n}_{m}, V\right)\right) .
$$

According to the proof of (1.20), $H_{0}\left(\mathfrak{n}_{m}, V\right)$ is $\mathfrak{m}_{m} \oplus \mathfrak{a}_{m}$-semisimple. Our assumption requires $H_{0}(\mathrm{r}, \hat{V})$ to be $\mathfrak{h}_{m}$-irreducible, hence (ii) $\Rightarrow$ (i).

In order to complete the proof, we establish a claim of independent interest:

$V$ is a UEP-module $\Leftrightarrow V^{\prime}$ is a UEP-module.

Using a result of D. Vogan [1, I.7.5], there exists an automorphism $\tau: G \rightarrow G$, preserving $K$, such that $V^{\tau} \cong V^{\prime}$; here, if $\pi$ is a globalization of $V$, then $\pi^{\tau}(x)=$ $\pi\left(\tau^{-1}(x)\right)$. This (real) automorphism defines a twisted minimal parabolic $\mathfrak{p}_{m}^{\tau}=\mathfrak{l}_{m}^{\tau} \oplus$ $\mathfrak{n}_{m}^{\tau}$. Now $H_{0}\left(\mathfrak{n}_{m}, V\right)$ is $\mathfrak{l}_{m}$-irreducible $\Leftrightarrow H_{0}\left(\mathfrak{n}_{m}^{\tau}, V^{\tau}\right)$ is $\mathfrak{l}_{m}^{\tau}$-irreducible. Conclude, $H_{0}\left(\mathfrak{n}_{m}^{\tau}, V^{\prime}\right)$ is $\mathfrak{l}_{m}^{\tau}$-irreducible. If we now twist back and consider all asymptotic expansions on $\left(\mathfrak{a}_{m, 0}\right)^{-}$, then $H_{0}\left(\mathfrak{n}_{m}, V^{\prime}\right)$ is $\mathfrak{l}_{m}$-irreducible. Reversing the roles of $V$ and $V^{\prime}$, noting $V^{\prime \prime} \cong V$, and using (1.20) establishes (1.26).

In order to prove (i) $\Rightarrow$ (ii), argue by contradiction: Suppose $H_{0}\left(\mathrm{n}_{m}, V\right)$ is $\mathrm{m}_{m} \oplus$ $a_{m}$-irreducible and $\hat{V}$ reduces; recall (1.20).

Case (1). $H^{0}\left(\mathrm{n}_{m}^{-}, \hat{V}\right)$ has two distinct minimal $a_{m}$-weights.

Let $\mu_{i}$ be two such weights. By the assumed minimality (and this is crucial!), one easily sees

$$
H^{0}\left(\mathrm{n}_{m}^{-}, \hat{V}\right)_{\mu_{i}} \cong H_{0}\left(\mathrm{n}_{m}, \hat{V}\right)_{\mu_{i}}
$$

as $\mathfrak{m}_{m} \oplus \mathfrak{a}_{m}$-modules. By (1.23), the right-hand side of (1.27) becomes $H_{0}\left(\mathfrak{n}_{m}, V\right)_{\mu_{i}}$; i.e. $H_{0}\left(\mathrm{n}_{m}, V\right)$ has two distinct $\mathrm{a}_{m}$-weights-contradiction.

In view of Case (1), we may as well assume $H^{0}\left(\mathrm{n}_{m}^{-}, \hat{V}\right)$ has precisely one minimal $a_{m}$-weight; call it $\nu$. By (1.24)(ii), choose a $\mathfrak{U}(\mathfrak{g})$-submodule $0 \neq Q \subseteq \hat{V}$ such that $\hat{V} / Q$ is nonzero irreducible in category $\left(\theta^{\prime}\right.$. There are two cases to consider.

Case (2). $H^{0}\left(n_{m}^{-}, Q\right)_{\nu} \neq 0$.

Again, we see that minimality will lead to (1.27) with $\mu_{i}$ replaced by $\nu$. The irreducibility of $H_{0}\left(\mathfrak{n}_{m}, V\right)$ shows $H_{0}\left(\mathfrak{n}_{m}, V\right) \cong H_{0}\left(\mathfrak{n}_{m}, V\right)_{\nu}$; hence $H^{0}\left(\mathfrak{n}_{m}^{-}, \hat{V}\right)_{\nu}$ is $m_{m} \oplus a_{m}$-irreducible via (1.23). Left exactness of cohomology yields the exact sequence

$$
0 \rightarrow H^{0}\left(\mathrm{n}_{m}^{-}, Q\right)_{\mu} \rightarrow H^{0}\left(\mathrm{n}_{m}^{-}, \hat{V}\right)_{\mu}
$$

for any $a_{m}$-weight $\mu$. Our hypothesis (for Case (2)) forces

$$
H^{0}\left(\mathrm{n}_{m}^{-}, Q\right)_{\nu}=H^{0}\left(\mathfrak{n}_{m}^{-}, \hat{V}\right)_{\nu} .
$$

Thus,

$$
Q \supseteq H^{0}\left(\mathrm{n}_{m}^{-}, Q\right)_{\nu}=H^{0}\left(\mathrm{n}_{m}^{-}, \hat{V}\right)_{\nu}=\hat{V}_{\nu}
$$


the last equality following since $\mathfrak{n}_{m}^{-} \cdot \hat{V}_{\nu}$ are vectors of $a_{m}$-weight lower than $\nu$. Conclude, $(\hat{V} / Q)_{\nu}=0$. On the other hand, $\hat{V} / Q$ is irreducible, so there exists $\mu \neq \nu$ such that $H_{0}\left(\mathfrak{n}_{m}, \hat{V} / Q\right)_{\mu} \neq 0$. The right exactness of $n_{m}$-homology leads to the exact sequence

$$
H_{0}\left(\mathrm{n}_{m}, \hat{V}\right)_{\mu} \rightarrow H_{0}\left(\mathrm{n}_{m}, \hat{V} / Q\right)_{\mu} \rightarrow 0 .
$$

By (1.23), $H_{0}\left(\mathfrak{n}_{m}, V\right)_{\mu}=H_{0}\left(\mathfrak{n}_{m}, \hat{V}\right)_{\mu} \neq 0$-contradiction.

Case (3). $H^{0}\left(\mathfrak{n}_{m}^{-}, Q\right)_{\nu}=0$.

According to [11] there exists a nondegenerate $g$-invariant pairing

$$
\left(V_{+}\right) \hat{)} \otimes\left(V_{-}^{\prime}\right) \hat{C} \text {. }
$$

Here, + (resp. - ) refers to defining asymptotic expansions on $\left(a_{m, 0}\right)^{-}$(resp. $\left.\left(\mathfrak{a}_{m, 0}\right)^{+}\right)$and asymptotic modules relative to ()$^{\left[\mathfrak{n}_{m}^{-}\right]}\left(\operatorname{resp} .()^{\left[\mathfrak{n}_{m}\right]}\right)$. Consequently, we have exhibited

$$
\left(V_{-}^{\prime}\right)^{\hat{n}}=\left[\left(V_{+}\right)^{\wedge}\right]_{\mathfrak{a}_{m} \text {-finite }}^{*}
$$

This fact, and an argument using the standard complex, shows

$$
\begin{aligned}
& H_{0}\left(\mathrm{n}_{m}^{-},\left(V_{-}^{\prime}\right)^{\hat{n}}\right) \cong H_{0}\left(\mathrm{n}_{m}^{-},\left[\left(V_{+}\right)^{\wedge}\right]_{\mathrm{a}_{m} \text {-finite }}^{*}\right) \\
& \cong H_{0}\left(\mathrm{n}_{m}^{-},\left(\left(V_{+}\right)^{\wedge}\right) *\right) \cong H^{0}\left(\mathrm{n}_{m}^{-},\left(V_{+}\right)^{\wedge}\right)^{*} .
\end{aligned}
$$

As in (1.28), $H^{0}\left(\mathrm{n}_{m}^{-}, Q\right)_{\eta} \hookrightarrow H^{0}\left(\mathrm{n}_{m}^{-},\left(V_{+}\right) \hat{~}_{\eta}\right.$. By the hypothesis of Case (3),

$$
H^{0}\left(\mathfrak{n}_{m}^{-}, Q\right)_{\mu} \neq 0 \text { for some } \mu \neq \nu \text {. }
$$

Thus, $H^{0}\left(\mathrm{n}_{m}^{-},\left(V_{+}\right)\right)_{\mu} \neq 0$ and $H^{0}\left(\mathrm{n}_{m}^{-},\left(V_{+}\right) \hat{)}_{\nu} \neq 0\right.$. Dualizing (1.30):

$$
H_{0}\left(\mathfrak{n}_{m}^{-},\left(V_{-}^{\prime}\right)^{\hat{n}}\right)_{-\mu} \neq 0 \text { and } H_{0}\left(\mathfrak{n}_{m}^{-},\left(V_{-}^{\prime}\right)^{\wedge}\right)_{-\nu} \neq 0 \text {. }
$$

Conclude, $H_{0}\left(n_{m}^{-}, V^{\prime}\right)$ is not irreducible (this requires the analog of (1.23) for asymptotics on $\left.\left(a_{m, 0}\right)^{+}\right)$. Twisting back to view asymptotic expansions on $\left(a_{m, 0}\right)^{-}$, we have $H_{0}\left(n_{m}, V^{\prime}\right)$ not irreducible - a contradiction to (1.26). Q.E.D.

(1.31) EXAMPLE. With $G$ as in (1.25), irreducible finite-dimensional $G$-modules are UEP-modules.

This follows from (1.25), since $F \cong \hat{F}$ for any finite-dimensional module $F$.

The following terminology will occur frequently in the sequel: Let $V$ be a UEP-module for $G$. Then $H_{0}\left(\mathfrak{n}_{m}, V\right)$ is an irreducible (finite-dimensional) $\mathrm{m}_{m} \oplus \mathfrak{a}_{m}$ module; (1.20). Consequently, $H_{0}\left(\mathfrak{n}_{m}, V\right)$ has a lowest $\mathfrak{h}_{m}$-weight. By this we will always mean the highest $\mathfrak{h}_{m}$-weight of $H_{0}\left(\mathfrak{n}_{m}, V\right)$ relative to the positive root system $\left[-\Phi^{+}\left(\mathfrak{g}, \mathfrak{h}_{m}\right)\right] \cap \Phi\left(\mathfrak{m}_{m} \oplus \mathfrak{a}_{m} \mathfrak{h}_{m}\right)$.

2. Infinitesimal character considerations. In this section we carry out an "infinitesimal reduction" of the UEP-problem. Throughout, $G$ is a connected semisimple real matrix group and all parabolic subgroups satisfy (1.11).

(2.1) THEOREM. If $V$ is a $U E P$-module for $G$, then $V$ has regular integral infinitesimal character $\chi_{\mathfrak{g}, \gamma} ;$ i.e. $2(\gamma \mid \alpha) /(\alpha \mid \alpha) \in \mathbf{Z} \backslash 0$ for all $\alpha \in \Phi\left(\mathfrak{g}, \mathfrak{h}_{m}\right)$. 
The basic strategy in proving (2.1) is laced throughout the entire paper. Consequently, we begin by sketching the argument. Suppose $R$ is a subset of the simple $\Phi^{+}\left(\mathfrak{g}, \mathfrak{a}_{m}\right)$ roots and denote

$$
\langle R\rangle=\text { root system generated by } R \text { in } \Phi\left(\mathrm{g}, \mathrm{a}_{m}\right) \text {. }
$$

Form parabolics

$$
\mathfrak{p}_{0}^{R}=\mathfrak{m}_{0}^{R} \oplus \mathfrak{a}_{0}^{R} \oplus \mathfrak{n}_{0}^{R} \quad \text { and } \quad P^{R}=M^{R} A^{R} N^{R},
$$

where

$$
\mathrm{m}_{0}^{R} \oplus \mathfrak{a}_{0}^{R}=\mathfrak{m}_{m, 0} \oplus \mathfrak{a}_{m, 0} \oplus \sum_{\beta \in\langle R\rangle} \mathfrak{g}_{\beta}, \quad \mathfrak{n}_{0}^{R}=\sum_{\beta \in \Phi^{+}\left(\mathfrak{g}, \mathfrak{a}_{m}\right) \backslash\langle R\rangle} \mathfrak{g}_{\beta}
$$

and $\mathfrak{g}_{\beta}=\beta$-root space. In case $R=\{\alpha\}$, we call these next to minimal parabolics. Observe that $V \hookrightarrow I_{P^{\alpha}}^{G}\left(U_{\alpha}\right), U_{\alpha}$ an irreducible quotient of $H_{0}\left(\mathfrak{n}^{\alpha}, V\right)$; this is (1.14). Next, $U_{\alpha}$ is a UEP-module for the real rank one Levi factor $M^{\alpha} A^{\alpha}$; cf. (2.3). These observations allow a "reduction to rank one" once we know something about UEP-modules for real rank one groups. With an eye toward proving (2.1), what we need in the real rank one setting will follow from results on reducibility of generalized principal series.

The following sequence of three lemmas - taken collectively - allows one to pass from a UEP-module for $G$ to a UEP-module for the simple real rank one piece of the Levi factor of a next to minimal parabolic.

(2.3) LEMMA. Let $V$ be a $U E P$-module for $G$ and $P=M A N$ a parabolic subgroup of $G$; recall (1.11). Let $Q$ (resp. $Q^{0}$ ) be an irreducible quotient of $H_{0}(\mathrm{n}, V)$ as a Harish-Chandra module for $M A\left(\right.$ resp. $\left.M^{0} A\right)$. Then $Q\left(\right.$ resp. $\left.Q^{0}\right)$ is a UEP-module for $M A\left(\operatorname{resp} . M^{0} A\right)$.

Proof. We take $\mathfrak{p}_{m} \cap(\mathfrak{m} \oplus \mathfrak{a})=\mathfrak{m}_{m} \oplus \mathfrak{a}_{m} \oplus\left(n_{m} \cap \mathfrak{m}\right)$ to be the minimal parabolic of $m \oplus a$. Right exactness of homology and (1.17) lead us to the exact sequence of $M_{m} A_{m}$-modules

$$
H_{0}\left(\mathrm{n}_{m}, V\right) \cong H_{0}\left(\mathrm{n}_{m} \cap \mathrm{m}, H_{0}(\mathrm{n}, V)\right) \stackrel{\phi}{\rightarrow} H_{0}\left(\mathfrak{n}_{m} \cap \mathrm{m}, Q\right) \rightarrow 0 .
$$

By the assumed irreducibility of $H_{0}\left(\mathfrak{n}_{m}, V\right), \phi$ is an isomorphism. Conclude that $Q$ is an irreducible Harish-Chandra module with irreducible zero homology. If $V$ has infinitesimal character $\chi_{g, \gamma}$, then $Q$ has infinitesimal character $\chi_{m \in a, \omega \gamma+\rho-\rho_{m \oplus a}}$ for some $\omega \in W\left(\mathfrak{g}, \mathfrak{h}_{m}\right)[10,(3.32)] . Q$ is a UEP-module once we observe $\omega \gamma+\rho-\rho_{\mathfrak{m} \oplus \mathfrak{a}}$ is $\Phi\left(\mathfrak{m} \oplus \mathfrak{a}, \dot{\mathfrak{h}}_{m}\right)$-regular. This is a consequence of the following fact (which we will need later):

$$
\begin{aligned}
& \text { \{simple } \left.\Phi^{+}\left(\mathfrak{m} \oplus \mathfrak{a}, \mathfrak{h}_{m}\right) \text { roots }\right\} \\
& \quad=\left\{\text { simple } \Phi^{+}\left(\mathfrak{g}, \mathfrak{h}_{m}\right) \text { roots vanishing on } a\right\} .
\end{aligned}
$$

(If $\alpha$ is $\Phi^{+}\left(\mathfrak{m} \oplus \mathfrak{a}, \mathfrak{h}_{m}\right)$ simple and $\alpha=\beta_{1}+\beta_{2}, \beta_{i} \in \Phi^{+}\left(\mathfrak{g}, \mathfrak{h}_{m}\right)$, then $\left.\beta_{1}\right|_{a}=$ $-\left.\beta_{2}\right|_{a}$. This contradicts compatibility remarks following (1.4). Conversely, any simple $\Phi^{+}\left(\mathfrak{g}, \mathfrak{h}_{m}\right)$ root which vanishes on $\mathfrak{a}$ is clearly $\Phi^{+}\left(\mathfrak{m} \oplus \mathfrak{a}, \mathfrak{h}_{m}\right)$ simple. $)$ Now, 
choose dual roots $\lambda_{i} \in \mathfrak{h}_{m}^{*}$ such that $2\left(\lambda_{i} \mid \alpha_{j}\right) /\left(\alpha_{j} \mid \alpha_{j}\right)=\delta_{i j}$, for $\alpha_{1}, \ldots, \alpha_{n}$ an enumeration of the simple $\Phi^{+}\left(g, \mathfrak{h}_{m}\right)$ roots. Using (2.4), we may suppose $\rho=\sum_{i=1}^{n} \lambda_{i}$ and $\rho_{\mathfrak{m} \oplus \mathfrak{a}}=\sum_{i=1}^{k} \lambda_{i}$, some $1 \leqslant k \leqslant n$. Now, $\left(\rho-\rho_{\mathfrak{m} \oplus \mathfrak{a}}\right) \perp \Phi\left(\mathfrak{m} \oplus \mathfrak{a}, \mathfrak{h}_{m}\right)$ and the $\Phi\left(\mathfrak{m} \oplus \mathfrak{a}, \mathfrak{h}_{m}\right)$-regularity of $\chi_{\mathfrak{m} \oplus \mathfrak{a}, \omega \gamma+\rho-\rho_{\mathfrak{m} \oplus \mathfrak{a}}}$ follows. The proof for $Q^{0}$ is identical. Q.E.D.

(2.5) LeMma. Let $P=M A N$ be a parabolic subgroup and $Q^{0}$ a UEP-module for $M^{0} A$. Denote by $M_{\mathrm{der}}^{0}$ the analytic subgroup of $M^{0} A$ corresponding to $[\mathrm{m}, \mathrm{m}]$. Then the restriction of $Q^{0}$ to $M_{\mathrm{der}}^{0}$ is a UEP-module for $M_{\mathrm{der}}^{0}$.

Proof. Since $\mathfrak{m} \oplus \mathfrak{a}=[\mathfrak{m}, \mathfrak{m}] \oplus$ (center), we clearly have $\left.Q^{0}\right|_{M_{\mathrm{der}}^{0}}$ irreducible as a Harish-Chandra $M_{\mathrm{der}}^{0}$-module. Apply (1.20). Q.E.D.

(2.6) Discussion. Because of our assumptions on $G$, it is always true that $G \hookrightarrow G_{\mathbf{C}}$. Although $G_{\mathbf{C}}$ is not necessarily simply connected, its universal cover $\tilde{G}_{\mathbf{C}-\text { has }}$ this property. The real points of $\tilde{G}_{\mathbf{C}}$, denoted $\tilde{G}$, form a connected semisimple real matrix group. The group $\tilde{G}$ is a finite covering of $G$ and $\tilde{G}=\tilde{G}_{1} \times \cdots \times \tilde{G}_{n}, \tilde{G}_{i}$ simple. Using the projection $\tilde{G} \rightarrow G$, we may "lift" the UEP-modules of $G$ to irreducible Harish-Chandra modules for $\tilde{G}$. Lemma (1.20) makes it clear:

(2.7) The UEP-modules for $G$ are naturally included among the UEP-modules for $\tilde{G}$.

(2.8) Lemma. Assume $G$ embeds into its simply connected complexification and write $G=G_{1} \times \cdots \times G_{n}$, according to (2.6). If $V$ is a UEP-module for $G$, then we may realize $V$ as

$$
V_{1} \otimes V_{2} \otimes \cdots \otimes V_{n}
$$

where $V_{i}$ is a UEP-module for $G_{i}$ and the action of $\left(\mathfrak{g}_{1} \oplus \cdots \oplus \mathfrak{g}_{n}, K_{1} \times \cdots \times K_{n}\right)$ on (2.9) is coordinatewise.

Proof. For simplicity, assume $G=G_{1} \times G_{2}$ and write $P_{m, i}=M_{m, i} A_{m, i} N_{m, i}$ for the minimal parabolic subgroup of $G_{i}$. As is well known, we may realize $V \cong V_{1} \times V_{2}$ with $V_{i}$ an irreducible Harish-Chandra module for $G_{i}$. Finally, observe

$$
H_{0}\left(\mathrm{n}_{m}, V\right) \cong H_{0}\left(\mathrm{n}_{m, 1}, V_{1}\right) \otimes H_{0}\left(\mathrm{n}_{m .2}, V_{2}\right) \text {. Q.E.D. }
$$

The final ingredient needed to prove (2.1) is some information about UEP-modules for real rank one groups.

(2.11) LEMMA. If $G$ is a connected simple real rank one matrix group and $I_{P_{m}}^{G}(U) a$ reducible generalized principal series with regular infinitesimal character $\chi_{\mathfrak{g} . \gamma}$, then

$$
2(\gamma \mid \alpha) /(\alpha \mid \alpha) \in \mathbf{Z} \text { for all } \alpha \in \Phi\left(\mathfrak{g}, \mathfrak{h}_{m}\right) .
$$

Proof. For the classical groups, see $[15,18,24,33]$. the case of $F_{4(-20)}$ proceeds as in [15], via [23]. Q.E.D.

(2.12) COROLlaRY. Theorem (2.1) holds whenever $G$ has real rank one.

(2.13) Proof of (2.1). Let $V$ be a UEP-module for $G$ with infinitesimal character $\chi_{\mathfrak{g} . \gamma}$. We begin by establishing:

(2.14) Let $P=M A N$ be a real parabolic ((1.11)) and $Q^{0}$ an irreducible quotient of $H_{0}(\mathrm{n}, V)$ as a Harish-Chandra module for $M^{0} A$. Then $Q^{0}$ has infinitesimal character $\chi_{\mathfrak{m} \oplus \mathfrak{a}, \tilde{\omega} \gamma+\rho-\rho_{\mathrm{m} \oplus \mathfrak{a}}}$ for some $\tilde{\omega} \in W\left(\mathfrak{g}, \mathfrak{h}_{m}\right)$, and $\tilde{\omega}$ depends only on $\gamma$. 
First, by (1.20), we know $H_{0}\left(\mathrm{n}_{m}, V\right)$ is $\mathrm{m}_{m} \oplus \mathrm{a}_{m}$-irreducible. By [10,(3.32)]:

$$
\begin{aligned}
& H_{0}\left(\mathrm{n}_{m}, V\right) \text { has infinitesimal character } \chi_{\mathfrak{m}_{m} \oplus \mathfrak{a}_{m}, \tilde{\omega} \gamma+\rho-\rho_{\mathfrak{w}_{m} \oplus \mathfrak{n}_{m}}} \\
& \text { for some } \tilde{\omega} \in W\left(\mathrm{~g}, \mathfrak{h}_{m}\right) .
\end{aligned}
$$

Next, assume $Q^{0}$ has infinitesimal character $\chi_{\mathfrak{m} \oplus a, \gamma^{\prime}}$ Then [10,(3.32)]:

$$
\begin{aligned}
& H_{0}\left(\mathrm{n}_{m} \cap \mathfrak{m}, Q^{0}\right) \text { has infinitesimal character } \chi_{\mathfrak{m}_{m} \oplus \mathfrak{a}_{m}, s \gamma^{\prime}+\rho_{m \oplus \mathfrak{n}}-\rho_{\mathrm{m}_{m} \oplus \mathfrak{a}_{m}}} \\
& \text { for some } s \in W\left(\mathfrak{m} \oplus \mathfrak{a}, \mathfrak{h}_{m}\right) .
\end{aligned}
$$

In view of (1.17),

$$
H_{0}\left(\mathrm{n}_{m} V\right) \cong H_{0}\left(\mathrm{n}_{m} \cap \mathrm{m}, H_{0}(\mathrm{n}, V)\right) \rightarrow H_{0}\left(\mathrm{n}_{m} \cap \mathrm{m}, Q^{0}\right) \rightarrow 0
$$

is exact, forcing the infinitesimal characters in (2.15) and (2.16) to agree. Using (1.13) we arrive at the formula

$$
\eta\left(\tilde{\omega} \gamma+\rho-\rho_{\mathfrak{m}_{m} \oplus a_{m}}\right)=s \gamma^{\prime}+\rho_{\mathfrak{m} \oplus a}-\rho_{\mathfrak{m}_{m} \oplus a_{m}}
$$

for some $\eta \in W\left(\mathfrak{m}_{m} \oplus \mathfrak{a}_{m}, \mathfrak{h}_{m}\right)$. According to (2.4), $W\left(m_{m} \oplus a_{m}, \mathfrak{h}_{m}\right)$ is generated by reflections about simple $m_{m}$-roots, each of which is orthogonal to $\rho-\rho_{m_{m} \oplus a_{m}}$ (as argued in the proof of (2.3)). Thus, $\eta\left(\tilde{\omega} \gamma+\rho-\rho_{\mathfrak{m}_{m} \oplus a_{m}}\right)=\eta \tilde{\omega} \gamma+\rho-\rho_{\mathfrak{m}_{m} \oplus a_{m}}$ and (2.17) leads us to

$$
\eta \tilde{\omega} \gamma+\rho-\rho_{\mathrm{m} \oplus \mathrm{a}}=s \gamma^{\prime} .
$$

Repeating the argument between (2.17) and (2.18) shows that

$$
\tilde{\omega} \gamma+\rho-\rho_{\mathrm{m} \oplus a}=\eta^{-1} s \gamma^{\prime} .
$$

Now, (2.14) follows from (2.19), (1.13) and the observation that $W\left(m_{m} \oplus a_{m}, \mathfrak{h}_{m}\right)$ $\subseteq W\left(\mathfrak{m} \oplus \mathfrak{a}, \mathfrak{h}_{m}\right)$.

For definiteness enumerate the simple $\Phi^{+}\left(\mathfrak{g}, \mathfrak{h}_{m}\right)$ roots as $\alpha_{1}, \ldots, \alpha_{k}, \alpha_{k+1}, \ldots, \alpha_{n}$, requiring that:

(2.20)(i) $B=B_{m} \cup B_{0}$, where $B=\left\{\alpha_{1}, \ldots, \alpha_{n}\right\}, \quad B_{m}=\left\{\alpha_{1} \cdots \alpha_{k}\right\}=$ simple $\Phi^{+}\left(\mathrm{m}_{m} \oplus \mathfrak{a}_{m}, \mathfrak{h}_{m}\right)$ roots and $B_{0}=\left\{\alpha_{k+1}, \ldots, \alpha_{n}\right\}=$ the remaining roots in $B ;$ (2.4).

(ii) Let $\bar{B}_{0}$ denote the restrictions $\left.\alpha_{j}\right|_{a_{m}}(k+1 \leqslant j \leqslant n)$. Then $\bar{B}_{0}=$ simple $\Phi^{+}\left(g, a_{m}\right)$ roots $[12$, p. 531$]$.

To prove (2.1), it is certainly enough to show $2\left(\tilde{\omega} \gamma \mid \alpha_{i}\right) /\left(\alpha_{i} \mid \alpha_{i}\right) \in \mathbf{Z}$ for $1 \leqslant i \leqslant n$, with $\tilde{\omega}$ as in (2.14). This is automatic for $1 \leqslant i \leqslant k$. Thus, we are reduced to showing

$$
2\left(\tilde{\omega} \gamma \mid \alpha_{j}\right) /\left(\alpha_{j} \mid \alpha_{j}\right) \in \mathbf{Z} \text { for } k+1 \leqslant j \leqslant n .
$$

Fix $j$ with $k+1 \leqslant j \leqslant n$. By (2.20)(ii), $\left.\alpha_{j}\right|_{a_{m}}$ is a simple restricted $a_{m}$-root, and therefore determines next to minimal parabolics $P_{j}=M_{j} A_{j} N_{j} \supseteq P_{m}$ and $\mathfrak{p}_{j}=\mathfrak{m}_{j} \oplus$ $\mathfrak{a}_{j} \oplus \mathfrak{n}_{j} \supseteq \mathfrak{p}_{m}$. Let $Q_{j}^{0}$ be an irreducible quotient of $H_{0}\left(\mathfrak{n}_{j}, V\right)$ as a Harish-Chandra $M_{j}^{0} A$-module. By (2.14), $Q_{j}^{0}$ has infinitesimal character $\chi_{\mathfrak{m}_{j} \oplus \mathrm{a}_{j} \tilde{\omega} \gamma+\rho-\rho_{j}}$, with $\tilde{\omega}$ depending only on $\gamma$ and $\rho_{\mathfrak{m}_{j} \oplus \mathfrak{a}_{j}}=\rho_{j}$ for notational simplicity. Lemmas (2.3) and (2.5) show that we may view $Q_{j}^{0}$ as a UEP-module for $M_{j \text {,der }}^{0}$ with infinitesimal character parametrized by $\tilde{\omega} \gamma+\rho-\rho_{j}$. Assume for the moment that $M_{j, \text { der }}^{0}$ embeds into its simply connected complexification. Since $M_{j \text {,der }}^{0}$ has real rank one, (2.8) shows $Q_{j}^{0} \cong W_{j} \otimes S_{j}$, where $W_{j}$ is an irreducible representation of the compact ideals 
(if any) in [ $\mathrm{m}, \mathrm{m}]$ and $S_{j}$ is a UEP-module for a connected real rank one simple matrix group. Notice that $W_{j}$ has integral infinitesimal character and, by (2.12), the same is true for $S_{j}$. Conclude that $\tilde{\omega} \gamma+\rho-\rho_{j}$ is integral with respect to $\Phi\left(\mathfrak{m}_{j} \oplus \mathfrak{a}_{j}, \mathfrak{h}_{m}\right)$. In case $M_{j, \text { der }}^{0}$ does not embed into its simply connected complexification, envoke (2.7) to see we still have

$$
2\left(\tilde{\omega} \gamma+\rho-\rho_{j} \mid \alpha\right) /(\alpha \mid \alpha) \in \mathbf{Z} \quad \text { for all } \alpha \in \Phi\left(m_{j} \oplus \mathfrak{a}_{j}, \mathfrak{h}_{m}\right) \text {. }
$$

As argued in the proof of (2.3), $\left(\rho-\rho_{j}\right) \perp \Phi\left(\mathfrak{m}_{j} \oplus \mathfrak{a}_{j}, \mathfrak{h}_{m}\right)$. Now, (2.22) yields (2.21) for our fixed $j$. Vary $j$, noting $\tilde{\omega}$ remains fixed. Q.E.D.

3. The role of the discrete series. In this section, we study the connection between UEP-modules and discrete series. As usual, $G$ is a connected semisimple real matrix group.

Let $V$ be a Harish-Chandra module for $G$. If $\left(\pi, V_{\pi}\right)$ is a globalization of $V$, the operator $\phi \rightarrow \int_{G} \pi(g) \phi(g) d g\left(\phi \in \mathbf{C}_{0}^{\infty}(G)\right)$ is of trace class and defines an invariant distribution (in the sense of Schwartz). This distribution, denoted $\Theta_{G}(V)$, is actually a locally $L^{1}$-function on $G$, real analytic on the set of regular points $G^{\prime} \subseteq G$ [5]. Furthermore, the definition of $\Theta_{G}(V)$ is independent of the globalization. If $U$ is another Harish-Chandra module, $\Theta_{G}(V)=\Theta_{G}(U) \Leftrightarrow V_{s s} \cong U_{s s}$, where $(\cdots)_{s s}$ denotes the direct sum of the irreducible subquotients in a composition series for $(\cdots)$. By an irreducible character, we understand a character of an irreducible HarishChandra module. A virtual character is a finite integral combination of irreducible characters.

According to Harish-Chandra's criteria [6], $G$ has a nonempty discrete series exactly when $G$ contains a compact Cartan subgroup $H^{c}$. In this case, the discrete series can be parametrized in terms of a certain lattice. To be precise, let $\Lambda_{\mathrm{ch}}=$ lattice of differentials of characters of $H^{c}$. Notice, $\Lambda_{\mathrm{ch}} \subseteq i\left(\mathfrak{h}_{0}^{c}\right)^{*}$. Put $\Phi\left(\mathfrak{g}, \mathfrak{h}^{c}\right)=$ root system of $\mathfrak{h}^{c}$ in $\mathfrak{g}$ and fix a positive system $\Phi^{+}\left(\mathfrak{h}^{c}\right)$. Form the corresponding half-sum of positive roots $\delta$, and $W\left(G, H^{c}\right)=N_{G}(H) / H$. Harish-Chandra's fundamental result on the discrete series is [6]:

(3.1) THeOREM. To each $\gamma \in \Lambda_{\mathrm{ch}}+\delta$ such that $(\gamma \mid \alpha)>0$ for all $\alpha \in \Phi^{+}\left(\mathfrak{h}^{c}\right)$, there exists a unique tempered invariant eigendistribution $\Theta_{G}\left(\Phi^{+}\left(\mathfrak{h}^{c}\right), \gamma\right)$ such that

$$
\left.\Theta_{G}\left(\Phi^{+}\left(\mathfrak{h}^{c}\right), \gamma\right)\right|_{H^{c} \cap G^{\prime}}=(-1)^{q} \frac{\sum_{\omega \in W\left(G, H^{c}\right)} \varepsilon(\omega) e^{\omega \gamma}}{\prod_{\alpha \in \Phi^{+}\left(\mathfrak{h}^{c}\right)}\left(e^{\alpha / 2}-e^{-\alpha / 2}\right)},
$$

where $\varepsilon(\omega)=$ sign of $\omega, q=\frac{1}{2} \operatorname{dim}_{\mathbf{R}} G / K .^{1}$ Every such $\Theta_{G}\left(\Phi^{+}\left(\mathfrak{h}^{c}\right), \gamma\right)$ is a discrete series character for $G$, and, conversely, every discrete series character for $G$ arises as one of the $\Theta_{G}\left(\Phi^{+}\left(\mathfrak{h}^{c}\right), \gamma\right)$ for a suitable positive system $\Phi^{+}\left(\mathfrak{h}^{c}\right)$.

We recall the generalization of (3.1) to the case where $M$ is a group satisfying (1.1). Specifically, let $M$ arise from $P=M A N$, where $P$ is a cuspidal parabolic

\footnotetext{
'As it stands, the denominator in this formula may not make sense. This problem can be alleviated by passing to a linear cover.
} 
subgroup. Then $M$ contains a compact Cartan $T$. Fix a positive system $\Phi^{+}(\mathrm{t})$ and denote by $\delta_{m}$ the half-sum of positive roots. Define

$$
M^{+}=\left\{m \in M \mid \mathrm{Ad}_{m}: M^{0} \rightarrow M^{0} \text { is inner }\right\} .
$$

Observe that $M^{0} \subseteq M^{+} \subseteq M$ with $M^{+}$normal in $M$. In fact [14, §2]:

(3.3) $M^{+}=M^{0} \cdot \Gamma_{M}$, where $\Gamma_{M}$ is a finite group isomorphic to a product of $\mathbf{Z}_{2}$-groups. Further, we may take $\Gamma_{M} \subseteq M_{m}, \Gamma_{M}$ central in $M$ and $M_{m}^{+}=M_{m}$.

The discrete series for $M$ is obtained as follows: Let $\Theta_{M^{0}}\left(\Phi^{+}(\mathrm{t}), \gamma\right)$ be a discrete series on $M^{0}$ and $\chi$ a character of $\Gamma_{M}$ such that $\chi$ agrees with $e^{\gamma-\delta_{\mathrm{m}}}$ on $T \cap \Gamma_{M}$. (We remark, (3.1) easily extends to the case of connected reductive groups with compact center-such as $M^{0}$.) Form $\Theta_{M^{0}}\left(\Phi^{+}(t), \gamma\right) \otimes \chi$ on $M^{+}$and induce to obtain

$$
\operatorname{ind}_{M^{+}}^{M}\left(\Theta_{M^{0}}\left(\Phi^{+}(\mathrm{t}), \gamma\right) \otimes \chi\right) \stackrel{\text { def }}{=} \Theta_{M}\left(\Phi^{+}(\mathrm{t}), \gamma, \chi\right) \text {. }
$$

We then have Harish-Chandra's generalization of (3.1) (cf. [14, (1.7), (1.13)]):

(3.4) The characters $\Theta_{M}\left(\Phi^{+}(t), \gamma, \chi\right)$ exhaust the discrete series for $M$ and

$$
\left.\Theta_{M}\left(\Phi^{+}(\mathrm{t}), \gamma, \chi\right)\right|_{M^{0}}=\bigoplus_{i=1}^{n} \Theta_{M^{0}}\left(W_{i}\right), \quad n=\left|M / M^{+}\right|,
$$

$W_{i}$ discrete series on $M^{0}$.

(3.5) Lemma. Let $P=M A N$ be a cuspidal parabolic subgroup of $G, W$ a discrete series on $M$ and $\nu \in a^{*}$. If $M \neq M^{+}$, then $W \otimes e^{\nu}$ is not a UEP-module for $M A$.

Proof. We may choose $\mathfrak{p}_{m} \cap(m \oplus a)=m_{m} \oplus a_{m} \oplus\left(n_{m} \cap m\right)$ as the minimal parabolic of $\mathrm{m} \oplus \mathrm{a}$. Computation of $H_{0}\left(\mathrm{n}_{m} \cap \mathrm{m}, W \otimes e^{\nu}\right)$ as a $\mathrm{m}_{m} \oplus \mathrm{a}_{m}$-module depends only upon $\left.\left(W \otimes e^{\nu}\right)\right|_{M^{0} A}$. In view of the remarks contained in (3.4), conclude, as $\mathrm{m}_{m} \oplus \mathrm{a}_{m}$-modules,

$$
H_{0}\left(\mathrm{n}_{m} \cap \mathrm{m}, W \otimes e^{\nu}\right) \cong H_{0}\left(\mathrm{n}_{m} \cap \mathrm{m},\left.W\right|_{M^{0}}\right) \otimes e^{\nu} \cong\left[\bigoplus_{i=1}^{n} H_{0}\left(\mathrm{n}_{m} \cap \mathrm{m}, W_{i}\right)\right] \otimes e^{\nu}
$$

now apply (1.20). Q.E.D.

In a moment we will use (3.5) to classify UEP-modules for $\operatorname{SL}(3, \mathbf{R})$. However, this will also require Langlands classification - as will many later arguments. Recall the notion of a collection of regular Langlands data: a cuspidal parabolic subgroup $P=M A N \supseteq P_{m}$, a discrete series $W$ for $M$, and a $\nu \in \mathrm{a}^{*}$, such that $\operatorname{Re}(\nu \mid \alpha) \leqslant 0$ for all $\alpha \in \Phi^{+}(\mathfrak{g}, \mathfrak{a})$.

(3.6) Theorem (HaRish-Chandra, Langlands, Milicıc [27, §4]). Let the induced module $I_{P}^{G}\left(W \otimes e^{\nu}\right)$, attached to a collection of regular Langlands data, have regular infinitesimal character. Then $I_{P}^{G}\left(W \otimes e^{\nu}\right)$ contains a unique irreducible submodule, denoted $J_{P}^{G}\left(W \otimes e^{\nu}\right)$. Every irreducible Harish-Chandra module for $G$, with regular infinitesimal character, is isomorphic to some $J_{P}^{G}\left(W \otimes e^{\nu}\right)$. The isomorphism class of $J_{P}^{G}\left(W \otimes e^{\nu}\right)$ determines the regular Langlands data uniquely up to conjugacy by elements of $G$.

(3.7) LeMma. Let $V$ be a UEP-module for $G$ with $\hat{V}$ finite-dimensional. Then $V$ is finite dimensional. 
Proof. According to Casselman $[26,4.5]$, the canonical g-invariant pairing $V^{\prime} \otimes \hat{V}$ $\rightarrow \mathbf{C}$ is nondegenerate. Q.E.D.

(3.8) Lemma. Let $G$ be covered by $\operatorname{SL}(3, \mathbf{R})$. Then the UEP-modules for $G$ are precisely the irreducible finite-dimensional modules.

Proof. By (2.7) it is enough to prove the lemma for $G=\operatorname{SL}(3, \mathbf{R})$. Let $V$ be a UEP-module for $G$. Since $\operatorname{SL}(3, \mathbf{R})$ has no discrete series, (3.6) shows:

$$
V=J_{P}^{G}\left(W \otimes e^{\nu}\right)
$$

for some set of regular Langlands data with $P \neq G$. Indeed, $P$ is a minimal or maximal parabolic. Suppose $V=J_{P_{m}}^{G}\left(W \otimes e^{\nu}\right)$; then (3.6) and the fact that $G$ is split (i.e. $\left.\mathfrak{h}_{m}=\mathfrak{a}_{m}\right)$ will force $H_{0}\left(\mathfrak{n}_{m}, V\right)$ to have lowest $\mathfrak{h}_{m}$-weight $\nu+\rho$, where $(\nu \mid \alpha)<0$ for all $\alpha \in \Phi^{+}\left(\mathfrak{g}, \mathfrak{h}_{m}\right)$. By $(2.1) \nu$ is integral, hence $2(\nu+\rho \mid \alpha) /(\alpha \mid \alpha) \in-\mathbf{N}$ for all $\alpha \in \Phi^{+}\left(\mathfrak{g}, \mathfrak{h}_{m}\right)$. An application of (1.25) and (1.23) shows: $\hat{V}$ is an irreducible quotient of some Verma module of integral antidominant lowest weight $\nu+\rho$. Using lowest weight theory and (3.7): $\hat{V}=V$ is finite dimensional. Next, suppose $V=$ $J_{P}^{G}\left(W \otimes e^{\nu}\right)$, with $P$ a maximal parabolic. A computation shows: $M=\mathrm{SL}^{ \pm}(2, \mathbf{R})$, $M^{+}=M^{0}=\mathrm{SL}(2, \mathbf{R})$; cf. [30,1.4]. By (3.5) $W \otimes e^{\nu}$ is not a UEP-module for $M A-$ a contradiction to (2.3). Q.E.D.

(3.9) REMARK. The "Verma reformulation" in (1.25) enters the above proof-and much of what follows - in a crucial way.

(3.10) Proposition. The UEP-modules for a complex group $G$ are precisely the irreducible finite-dimensional modules.

Proof. $H_{0}\left(\mathfrak{n}_{m}, V\right)$ has lowest $\mathfrak{h}_{m}$-weight $\lambda$. Let $\alpha$ be a simple $\Phi^{+}\left(\mathfrak{g}, \mathfrak{a}_{m}\right)$ root. Recalling (2.2), form the next to minimal parabolic subgroup $P^{\alpha}=M^{\alpha} A^{\alpha} N^{\alpha}$ determined by $\{\alpha\}$. If $Q_{\alpha}$ is an irreducible Harish-Chandra quotient of $H_{0}\left(n^{\alpha}, V\right)$, then (1.17) and right exactness show

$$
H_{n}\left(\mathfrak{n}_{m}, V\right) \cong H_{0}\left(\mathfrak{n}_{m} \cap \mathrm{m}^{\alpha}, Q_{\alpha}\right) .
$$

Now, by (2.3), $Q_{\alpha}$ is a UEP-module for the real rank one group $M^{\alpha} A^{\alpha}$. The hereditary properties of complex groups will force $\left[\mathfrak{m}^{\alpha}, \mathfrak{m}^{\alpha}\right]=\mathfrak{g} \mathfrak{l}(2, \mathbf{C}) \oplus$ (compact ideal). Since $\mathfrak{S} \mathfrak{l}(2, \mathrm{C}) \cong \mathfrak{s} \mathfrak{o}(3,1),\left(\right.$ A.1.4) and the proof of $(2.1)$ show that $Q_{\alpha}$ is finite dimensional. The roots of $m^{\alpha} \oplus a^{\alpha}$ are given by $\Phi\left(\mathfrak{m}^{\alpha} \oplus \mathfrak{a}^{\alpha}, \mathfrak{h}_{m}\right)$. It follows from (3.11) that $2(\lambda \mid \beta) /(\beta \mid \beta) \in-\mathbf{N}$ for all $\beta \in \Phi^{+}\left(\mathfrak{m}^{\alpha} \oplus \mathfrak{a}^{\alpha}, \mathfrak{h}_{m}\right)$.

Let $\alpha$ vary over the simple $\Phi^{+}\left(\mathrm{g}, a_{m}\right)$ roots and obtain $2(\lambda \mid \beta) /(\beta \mid \beta) \in-\mathbf{N}$ for all $\beta \in \Phi^{+}\left(\mathfrak{g}, \mathfrak{h}_{m}\right)$ (this uses (2.20)(ii)!). As usual, (1.25), (1.23) and (3.7) show that $\hat{V}=V$ is finite dimensiona!. Q.E.D.

(3.12) Discussion. The classification of real simple groups will play an important role in the sequel. Consequently, we review a few relevant facts $[12, \S 10]$. First, the simple real groups fall into two classes-corresponding to $\mathrm{g}=\mathrm{g}_{0} \otimes \mathbf{C}$ being simple or a sum of two ideals. In the second case, $G$ is a complex group. By (3.10) the UEP-problem has been solved in this context. Consequently, the remainder of the paper is concerned with simple $\mathrm{g}$. In this case we can list all $\mathfrak{g}_{0}$ as follows: Recall from (2.20) that $B=B_{m} \cup B_{0}$ and $\bar{B}_{0}$. The classification data for $g_{0}$ consist of: (i) a 
Satake diagram of $B$, (ii) a Dynkin diagram of $\bar{B}_{0}$, and (iii) a multiplicity function $m$ : $\bar{B}_{0} \rightarrow \mathbf{N}$. Recall, (i) is just the Dynkin diagram of $B$ with "nodes" corresponding to $\alpha \in B_{m}$ denoted by "black dots", and two elements of $B_{0}$ which restrict to the same element of $\bar{B}_{0}$ are connected by a "curved arrow". The useful fact is:

The structure of the Levi factors of real parabolics $\mathfrak{\supseteq} \mathfrak{p}_{m}$ may be determined using the classification data.

The true meaning of this remark becomes clear in the proof of our next theorem. The technique employed there will resurface at later points in the paper. Consequently, we will be fairly explicit-later referring to the "proof of (3.13)".

(3.13) THEOREM. Let $G$ be a simple group with no discrete series. Then the $U E P$-modules for $G$ are precisely the irreducible finite-dimensional modules.

Proof. In view of (3.10) and (3.12), we may assume $G$ is noncomplex. As previously remarked, $G$ has discrete series $\Leftrightarrow \operatorname{rank} G=\operatorname{rank} K$. Using the classification of real forms [12,p. 518], one easily checks that $\mathfrak{g}_{0}$ is among the following algebras: $\mathfrak{S} \mathfrak{I}(n, \mathbf{R})(n \geqslant 3) ; \mathfrak{S L}^{*}(2 n)(n \geqslant 3) ; \mathfrak{s o}(p, q)(p, q$ odd $) ; \mathrm{e}_{6(6)} ;$ or $\mathrm{e}_{6(-26)}$. In (A.1.4), we prove the UEP-modules for groups covered by $\operatorname{SPIN}(2 n+1,1)$ are precisely finite dimensional. This fact, (2.7), (3.8) and the proof of (3.10) establish (recall (2.20)):

(3.14) Let $V$ be a UEP-module for $G$. Suppose that for every $\alpha \in B_{0}$ we can find a parabolic $P=M A N$ such that:

(i) $\alpha \in \Phi^{+}\left(m \oplus a, \mathfrak{h}_{m}\right)$;

(ii) $[\mathfrak{m}, \mathfrak{m}]_{0}=\mathfrak{g} \mathfrak{o}(2 n+1,1) \oplus$ (compact ideal) or $[\mathfrak{m}, \mathfrak{m}]_{0}=\mathfrak{g} \mathfrak{l}(3, \mathbf{R}) \oplus$ (compact ideal).

Then $V$ is finite dimensional.

For us, the parabolic $P$ in (3.14) will either be next to minimal or "second from minimal". (A second from minimal parabolic $\mathfrak{p}=\mathfrak{m} \oplus \mathfrak{a} \oplus \mathfrak{n}$ is determined by $R=\left\{\alpha_{1}, \alpha_{2}\right\}$ as in (2.2).) We prove (3.13) case-by-case. First, suppose $\mathrm{g}_{0}=\mathfrak{g} \mathfrak{l}(n, \mathbf{R})$ $(n \geqslant 3)$. In view of (3.8), assume $n \geqslant 4$. Form the sets $R_{i}=\left\{\beta_{i}, \beta_{i+1}\right\}$ for $1 \leqslant i \leqslant n$ - 2. For each $R_{i}$, let $\mathfrak{p}_{i}=\mathfrak{m}_{i} \oplus \mathrm{a}_{i} \oplus \mathrm{n}_{i}$ be the associated second from minimal parabolic. Observe that $\left[\mathfrak{m}_{i}, \mathfrak{m}_{i}\right]_{0}=\mathfrak{s} \mathfrak{l}(3, \mathbf{R})$; hence an application of $(3.14)$ yields (3.13).

TABLE $1 . \mathfrak{I l}(n, \mathbf{R})$

\begin{tabular}{|c|c|c|c|}
\hline Satake diagram of $B$ & Dynkin diagram of $\overline{\mathrm{B}}_{0}$ & $\mathrm{~m}_{B}$ & $\mathrm{~m}_{2 \beta}$ \\
\hline $\mathrm{O}-\mathrm{O} \cdot \cdots \rightarrow$ & $\mathrm{O}-\mathrm{O}-\cdots$ & 1 & 0 \\
\hline$\alpha_{1} \quad \alpha_{2}$ & $B_{1} \quad B_{2}$ & & \\
\hline
\end{tabular}


In case $\mathfrak{g}_{0}=e_{6(6)}$, the argument proceeds exactly as for $\mathfrak{g}_{0}=\mathfrak{s} \mathfrak{l}(n, \mathbf{R})(n \geqslant 4)$; we omit the details. Let $\mathfrak{g}_{0}=\mathfrak{s} \mathfrak{u}^{*}(2 n)(n \geqslant 3)$. Let $R_{i}=\left\{\beta_{2 i}\right\}$ for $i \leqslant i \leqslant n-1$ and form the next to minimal parabolic $\mathrm{p}_{i}=\mathrm{m}_{i} \oplus \mathrm{a}_{i} \oplus \mathrm{n}_{i}$ determined by $R_{i}$. Using Tables 2 and 6 , one can see that $\left[m_{i}, m_{i}\right]_{0}=\mathfrak{s} \mathfrak{v}(5,1) \oplus$ (compact ideal); apply (3.14).

$$
\text { TABLE 2. } \because u^{*}(2 n) \quad(n \geqslant 3)
$$

\begin{tabular}{lllllllll}
\hline Satake diagram of $B$ & Dynkin diagram of $\overline{\mathrm{B}}_{0}$ & $\mathrm{~m}_{\beta}$ & $\mathrm{m}_{2 \beta}$ \\
\hline$\alpha_{1}$ & $\alpha_{2}$ & $\alpha_{3}$ & $\alpha_{2 n-1}$ & $\beta_{2}$ & $\beta_{4}$ & $\beta_{2 n-2}$ & & 0 \\
\hline
\end{tabular}

In the case $\mathfrak{g}_{0}=\mathfrak{e}_{6(-26)}$, the argument proceeds exactly as for $\mathfrak{s} \mathfrak{u}^{*}(2 n)$. (In this case the $\mathfrak{p}_{i}$ will have $\left[\mathfrak{m}_{i}, \mathfrak{m}_{i}\right]_{0}=\mathfrak{s} \mathfrak{o}(9,1) \oplus$ (compact ideal).)

Finally, it remains to consider $\mathfrak{g}_{0}=\mathfrak{s} \mathfrak{o}(p, q)$, with $1 \leqslant p \leqslant q$ and $p, q \in 2 \mathbf{N}+1$. If $p=1,(3.13)$ is a consequence of (A.1.4). Assume $3 \leqslant p \leqslant q$ and $p, q$ are odd.

TABLE 3. $\check{o}(p, q) \quad(1<p \leqslant q ; p, q$ odd $)$

\begin{tabular}{llllll}
\hline Satake diagram of $B \quad$ Dynkin diagram of $\bar{B}_{0}$ & $m_{B}$ & $m_{2 \beta}$ \\
\hline
\end{tabular}
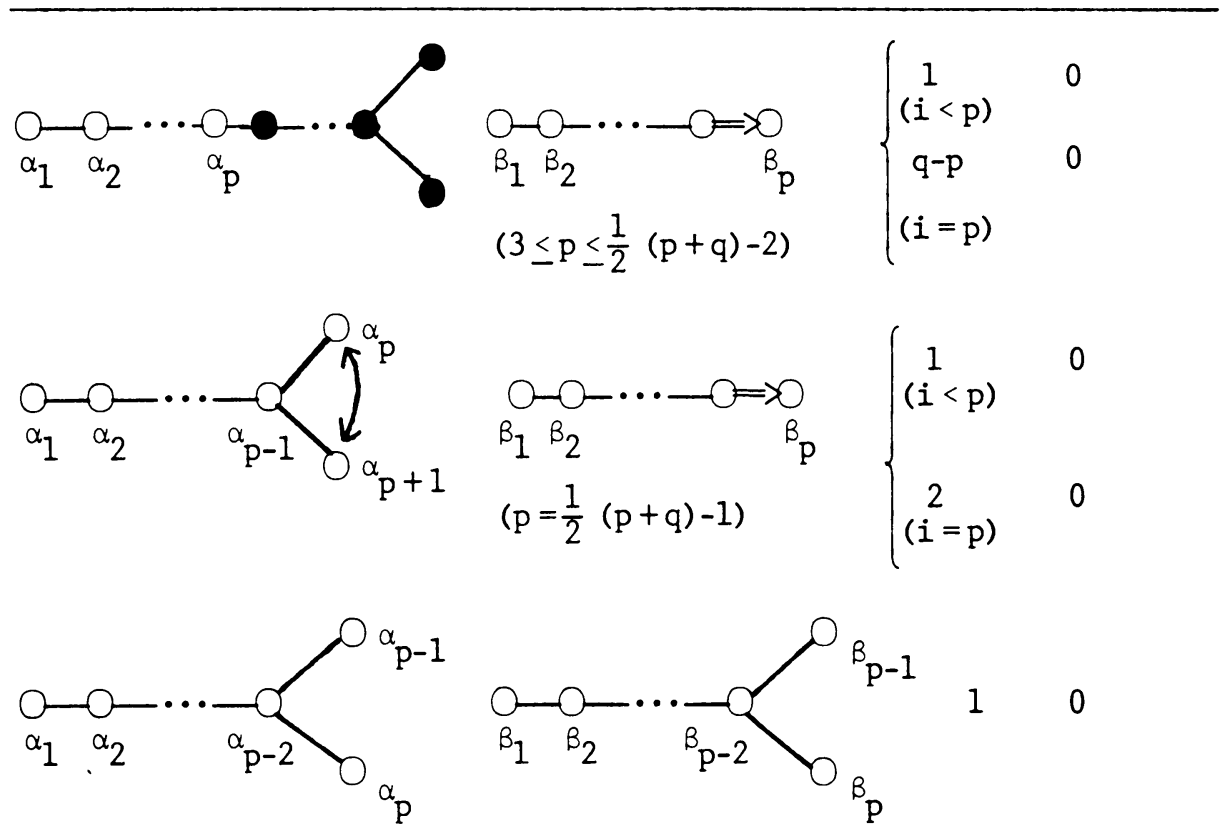
The case when $p=q$ is handled just like $\mathfrak{g}_{0}=\mathfrak{g l}(n, \mathbf{R})(n \geqslant 4)$. Assume $p=$ $\frac{1}{2}(p+q)-1$ - the quasi-split case. Let $R_{i}=\left\{\beta_{i}, \beta_{i+1}\right\}$ for $1 \leqslant i \leqslant p-2$ and $R=\left\{\beta_{p}\right\}$. Let $\mathfrak{p}_{i}=\mathrm{m}_{i} \oplus \mathrm{a}_{i} \oplus \mathrm{n}_{i}$ be the second from minimal parabolics determined by $R_{i}$, and $\mathfrak{p}=\mathrm{m} \oplus \mathrm{a} \oplus \mathrm{n}$ the next to minimal parabolic corresponding to $R$. By Tables 1,3 and $6,\left[\mathfrak{m}_{i}, \mathfrak{m}_{i}\right]_{0}=\mathfrak{s} \mathfrak{l}(3, \mathbf{R}) \oplus$ (compact ideal $)$ and $[\mathfrak{m}, \mathfrak{m}]_{0}=\mathfrak{s} \mathfrak{l}(2, \mathbf{C}) \oplus$ (compact ideal). Recalling that $\mathfrak{s} \mathfrak{o}(3,1) \cong \mathfrak{s} \mathfrak{l}(2, \mathbf{C})$, we may apply (3.14) to establish (3.13). Finally, suppose $3 \leqslant p \leqslant \frac{1}{2}(p+q)-2$. Let $R_{i}$ and $R$ be just as above. Then $\left[\mathfrak{m}_{i}, \mathfrak{m}_{i}\right]_{0}=\mathfrak{g} \mathfrak{l}(3, \mathbf{R}) \oplus$ (compact ideal) and $[\mathfrak{m}, \mathfrak{m}]_{0}=\mathfrak{g} \mathfrak{o}(q-p+1,1) \oplus$ (compact ideal). Again, (3.14) leads to (3.13). Q.E.D.

(3.15) REMARK. If $G$ is a simple real form, then we now know that existence of discrete series is a necessary condition for having non-finite-dimensional UEP-modules. However, the condition is not sufficient; cf. (6.1).

We now turn toward establishing a closer link between discrete series and UEP-modules. Write $\Lambda_{m} \subseteq\left(\mathfrak{h}_{m}\right)^{*}$ for the lattice of weights of finite-dimensional representations of $G$. If $\mu \in \Lambda_{m}$, let $F_{\mu}$ be the irreducible finite-dimensional representation of $G$ with extremal weight $\mu[30,0.4]$. Let $\mu_{1}, \mu_{2} \in \Lambda_{m}$ and $\gamma \in\left(\mathfrak{h}_{m}\right)^{*}$. We define the Jantzen-Zuckerman translation functor $\psi_{\gamma+\mu_{2}}^{\gamma+\mu_{1}}$ on the category of HarishChandra modules $\mathcal{H}$, or on category $\mathcal{O}^{\prime}$, by the rule

$$
\psi_{\gamma+\mu_{2}}^{\gamma+\mu_{1}}(X)=P_{\gamma+\mu_{2}}\left(F_{\mu_{2}-\mu_{1}} \otimes P_{\gamma+\mu_{1}}(X)\right) .
$$

Here, for $\eta \in \mathfrak{h}_{m}^{*}$,

$$
P_{\eta}(X)=\left\{x \in X \text { : for some } n>0, \text { and all } Z \in 3(\mathfrak{g}),\left(Z-\chi_{\mathfrak{g}, \eta}(Z)\right)^{n} \cdot x=0\right\} .
$$

It is well known that $\psi_{\gamma+\mu_{2}}^{\gamma+\mu_{1}}$ is an exact covariant functor mapping $\mathcal{H}$ (resp. $\theta^{\prime}$ ) into itself; see [30, 4.5.8, 34].

Now suppose $\gamma$ is a regular integral element of $\left(\mathfrak{h}_{m}\right)^{*}$. We obtain a positive root system $\Phi^{+}(\gamma)=\left\{\alpha \in \Phi\left(\mathfrak{g}, \mathfrak{h}_{m}\right):(\gamma \mid \alpha)>0\right\}$ with simple roots $B(\gamma)$. If $\alpha \in B(\gamma)$ let $\lambda_{\alpha}$ be the fundamental weight dual to $\alpha$ and set $c=2(\gamma \mid \alpha) /(\alpha \mid \alpha)$. Observe that $\gamma-c \lambda_{\alpha}$ is dominant integral and regular with respect to all $B(\gamma)$ simple roots, except $\alpha$. For notational simplicity we set

$$
\psi_{\alpha}=\psi_{\gamma-c \lambda_{\alpha}}^{\gamma} \text { and } \phi_{\alpha}=\psi_{\gamma}^{\gamma-c \lambda_{\alpha}} \text {. }
$$

(Possibly passing to a finite linear cover of $G$, (3.17) always makes sense. In the future we often do this without further comment. In view of (2.7), the study of unique embedding modules will not be affected.) We will be interested in the functor $\phi_{\alpha} \psi_{\alpha}$. We know [34]: $\phi_{\alpha} \psi_{\alpha} X=0$ only if $\psi_{\alpha} X=0$. This leads to the (Borho-JantzenDuflo) $\tau$-invariant of $X$ :

$$
\tau(X)=\left\{\alpha \in B(\gamma): \psi_{\alpha} X=0\right\} .
$$

(3.18) THEOREM $[\mathbf{2 8}, 2.1]$. Let $X$ be an irreducible g-module with infinitesimal character $\chi_{\mathfrak{g}, \gamma}$. Assume $X$ is a lowest weight module or a Harish-Chandra module for some real form of $\mathrm{g}$. If $\alpha \notin \tau(X)$, then $\phi_{\alpha} \psi_{\alpha} X$ has $X$ as its unique irreducible submodule and irreducible quotient. The sequence

$$
0 \rightarrow X \stackrel{d_{1}}{\rightarrow} \phi_{\alpha} \psi_{\alpha} X \stackrel{d_{2}}{\rightarrow} X \rightarrow 0
$$


is a chain complex with cohomology $U_{\alpha} X=\operatorname{ker} d_{2} / \operatorname{Im} d_{1} . U_{\alpha} X$ has a finite composition series and $\alpha \in \tau\left(U_{\alpha} X\right)$.

We now state the Kazhdan-Lusztig conjectures in the form we need.

(3.19) THEOREM. In the setting of (3.18), if $\gamma$ is regular integral, then $U_{\alpha} X$ is a completely reducible g-module.

This result is a synthesis of work due to several people. For the precise distribution of credit see [29]. Actually, the Kazhdan-Lusztig conjectures refer to "formulas" for the multiplicities of "standard modules" in a given irreducible. However, these "formulas" are equivalent to complete reducibility of the $U_{\alpha}$ 's.

Now suppose $V$ is an irreducible Harish-Chandra module with regular integral infinitesimal character $\chi_{\mathrm{g}, \gamma}$. If $\alpha \notin \tau(V),(3.18)$ gives us the complex

$$
C^{*}: 0 \rightarrow V \stackrel{d_{1}}{\rightarrow} \phi_{\alpha} \psi_{\alpha} V \stackrel{d_{2}}{\rightarrow} V \rightarrow 0 .
$$

where $d_{1}$ is an injection, $d_{2}$ is a surjection and $U_{\alpha} V$ is the cohomology. By exactness of $(\cdots)((1.23))$, we obtain the complex

$$
D^{*}: 0 \rightarrow \hat{V} \stackrel{\hat{d}_{1}}{\rightarrow}\left(\phi_{\alpha} \psi_{\alpha} V\right)^{\wedge} \stackrel{\hat{d}_{2}}{\rightarrow} \hat{V} \rightarrow 0
$$

where $\hat{d}_{1}$ is injective, $\hat{d}_{2}$ is surjective and $\left(U_{\alpha} V\right) \hat{=} H\left(D^{*}\right)$. If $F$ is finite dimensional, there is a natural embedding $\hat{V} \otimes F \hookrightarrow(V \otimes F)$. Using the exactness of $(\cdots) \hat{)}$, one can argue (cf. [11])

$$
\hat{V} \otimes F \text { is naturally isomorphic to }(V \otimes F) \hat{.}
$$

The asymptotic functor clearly commutes with projections, so (3.20) leads us to the complex

$$
E^{*}: 0 \rightarrow \hat{V}_{1}^{d_{1}^{\prime}} \rightarrow \phi_{\alpha} \psi_{\alpha} \hat{V}^{d_{2}^{\prime}} \rightarrow \hat{V} \rightarrow 0
$$

where $\left(U_{\alpha} V\right)=H\left(D^{*}\right)=H\left(E^{*}\right)$. Our next result computes this cohomology, in a special case.

(3.21) Proposition. Let $V$ be an irreducible Harish-Chandra module with regular integral infinitesimal character. If $\hat{V}$ is completely reducible, then $\left(U_{\alpha} V\right) \hat{\text { is completely }}$ reducible.

(3.22) REMARK. In the course of proving (3.21), we actually show that $\alpha$ is not in the $\tau$-invariant of every summand in $\hat{V}$.

Proof of (3.21). Assume $\hat{V}=N_{1} \oplus \cdots \oplus N_{n}$ is a decomposition of $\hat{V}$ into irreducibles. Let $S=\left\{i: \alpha \notin \tau\left(N_{i}\right)\right\}$. $E^{*}$ now becomes

$$
E^{*}: 0 \rightarrow \bigoplus_{i=1}^{n} N_{i} \stackrel{d_{1}^{\prime}}{\rightarrow} \bigoplus_{S} \phi_{\alpha} \psi_{\alpha} N_{i} \stackrel{d_{2}^{\prime}}{\rightarrow} \bigoplus_{i=1}^{n} N_{i} \rightarrow 0,
$$

where $d_{1}^{\prime}$ is injective, $d_{2}^{\prime}$ is surjective. Fix $1 \leqslant i \leqslant n$. Then

$$
N_{i} \underset{\neq 0}{\stackrel{d_{1}}{\rightarrow}} \bigoplus_{S} \phi_{\alpha} \psi_{\alpha} N_{i} \stackrel{\text { proj }}{\rightarrow} \phi_{\alpha} \psi_{\alpha} N_{j(i)}
$$


for some $j(i) \in S$. Since $N_{i}$ is irreducible, $0 \rightarrow N_{i} \stackrel{d_{i}^{\prime}}{\rightarrow} \phi_{\alpha} \psi_{\alpha} N_{j(i)}$ is exact. We may apply (3.18) to $\phi_{\alpha} \psi_{\alpha} N_{j(i)}$, concluding that $N_{i}$ must be the unique irreducible submodule of $\phi_{\alpha} \psi_{\alpha} N_{j(i)}$. Now, repeat this argument for each $1 \leqslant i \leqslant n$. Using the fact $d_{1}^{\prime}$ is $1: 1$ and each $\phi_{\alpha} \psi_{\alpha} N_{j(i)}$ contains a unique irreducible submodule, we deduce $S=$ $\{1,2, \ldots, n\}$ - as remarked prior to the proof. $E^{*}$ now becomes

$$
E^{*}: 0 \rightarrow \bigoplus_{i=1}^{n} N_{i} \stackrel{d_{1}^{\prime}}{\rightarrow} \bigoplus_{i=1}^{n} \phi_{\alpha} \psi_{\alpha} N_{i} \stackrel{d_{2}^{\prime}}{\rightarrow} \bigoplus_{i=1}^{n} N_{i} \rightarrow 0 .
$$

Next we study $d_{2}^{\prime}$. Since $d_{2}^{\prime}$ is onto, it must be the case that there exists $i_{0}$ such that

$$
\phi_{\alpha} \psi_{\alpha} N_{i_{0}} \underset{\neq 0}{\stackrel{d_{2}^{\prime}}{\rightarrow}} \bigoplus_{i=1}^{n} N_{i} \underset{\neq 0}{\stackrel{\text { proj }}{\rightarrow}} N_{k\left(i_{0}\right)} .
$$

Since $N_{k\left(i_{0}\right)}$ is irreducible, $\phi_{\alpha} \psi_{\alpha} N_{i_{0}} \stackrel{d_{2}^{\prime}}{\rightarrow} N_{k\left(i_{0}\right)} \rightarrow 0$ is exact. Again, (3.18) applied to $\phi_{\alpha} \psi_{\alpha} N_{i_{0}}$ forces $N_{k\left(i_{0}\right)}$ to be the unique irreducible quotient of $\phi_{\alpha} \psi_{\alpha} N_{i_{0}}$. Continuing on, using the fact that $d_{2}^{\prime}$ is onto and (3.18), we naturally induce $n$ "coordinatewise complexes"

$$
E_{i}^{*}: 0 \rightarrow N_{i} \stackrel{d_{1}^{\prime}}{\rightarrow} \phi_{\alpha} \psi_{\alpha} N_{j(i)}^{\stackrel{d_{2}^{\prime}}{\rightarrow}} N_{k(j(i))} \rightarrow 0
$$

where $N_{j(i)} \cong N_{i} \cong N_{k(j(i))}$ is the unique irreducible submodule and quotient of $\phi_{\alpha} \psi_{\alpha} N_{j(i)}$. Conclude

$$
\left(U_{\alpha} V \hat{)}=H\left(E^{*}\right)=\bigoplus_{i=1}^{n} H\left(E_{i}^{*}\right)=\bigoplus_{i=1}^{n} U_{\alpha} N_{i} .\right.
$$

Now apply (3.19) to each $U_{\alpha} N_{i}$. Q.E.D.

Let $\gamma \in\left(\mathfrak{h}_{m}\right)^{*}$. A coherent family (based on $H_{m}$ and $\gamma+\Lambda_{m}$ ) is a collection of virtual characters $\left\{\Theta_{G}(\gamma+\nu)\right\}_{\nu \in \Lambda_{m}}$ which satisfies:

(3.25)(a) $\Theta_{G}(\gamma+\nu)$ has infinitesimal character $\chi_{\mathfrak{g}, \gamma+\nu}$.

(b) If $F$ is a finite-dimensional $G$-module, then

$$
F \otimes \Theta_{G}(\gamma+\nu)=\sum_{\eta \in \Delta(F)} \Theta_{G}(\gamma+(\nu+\eta)),
$$

where $\Delta(F)=$ weights of $F$ as an $\mathfrak{h}_{m}$-module (counting multiplicities).

Schmid and Zuckerman [30, 7.2] have shown:

(3.26) A virtual Harish-Chandra module $\Theta_{G}$ with infinitesimal character $\chi_{\mathfrak{g}, \gamma}$ can be placed in a coherent family such that $\Theta_{G}(\gamma+0)=\Theta_{G}$. If $\gamma$ is regular, then this family is unique.

Fix a regular infinitesimal character $\chi_{\mathfrak{g}, \gamma}$. We define a representation of $W\left(\mathfrak{g}, \mathfrak{h}_{m}\right)$ on the lattice of virtual characters with infinitesimal character $\chi_{\mathfrak{g}, \gamma}$ as follows:

$$
\omega \cdot \Theta_{G}=\Theta_{G}\left(\omega^{-1}(\gamma+0)\right) \text {. }
$$

According to [30, 7.2.29]: (i) $\omega \cdot \Theta_{G}$ is well defined; (ii) if $\omega_{1}, \omega_{2} \in W\left(g, \mathfrak{h}_{m}\right)$, then $\omega_{1} \cdot\left(\omega_{2} \cdot \Theta_{G}\right)=\left(\omega_{1} \omega_{2}\right) \cdot \Theta_{G}$; (iii) if $\left\{\Theta_{G}(\gamma+\nu)\right\}_{\gamma+\Lambda_{m}}$ is the coherent family based on $H_{m}$ and $\gamma+\Lambda_{m}$, and $\gamma+\nu$ is regular, then $\omega \cdot \Theta_{G}(\gamma+\nu)=\Theta_{G}\left(\omega^{-1}(\gamma+\nu)\right)$.

(3.28) Situation. Let $V$ be a UEP-module for $G$. By (1.25), (1.24)(ii) and (1.23), we know $\hat{V}=L(\gamma)$ and $H_{0}\left(\mathfrak{n}_{m}, V\right) \cong H_{0}\left(\mathfrak{n}_{m}, \hat{V}\right)$ has lowest $\mathfrak{h}_{m}$-weight $\gamma+\rho$. We see $V$ 
has infinitesimal character $\chi_{\mathfrak{g} . \gamma}$. Let $\bar{\omega} \in W\left(\mathfrak{g}, \mathfrak{h}_{m}\right)$ such that $(\bar{\omega} \gamma \mid \alpha)<0$ for all $\alpha \in \Phi^{+}\left(\mathfrak{g}, \mathfrak{h}_{m}\right)$. Now, $\bar{\omega} \gamma-\gamma=-\mu$ is a positive integral combination of negative roots. Let $F_{-\mu}$ be the finite-dimensional representation of lowest weight $-\mu$, leading to the Harish-Chandra module $V \otimes F_{-\mu}$ and the category $\theta^{\prime}$ module $\hat{V} \otimes F_{-\mu}$. Since $(\cdots)$ is exact $((1.23))$, every irreducible subquotient $Q$ of $V \otimes F_{-\mu}$ has $\hat{Q}$ a subquotient of $\left(V \otimes F_{-\mu}\right)$. A priori, $\hat{Q}$ is not irreducible!. Using $(2.1)$ and [17, p. 78]:

(*) $P_{\gamma}\left(V \otimes F_{-\mu}\right)$ contains an irreducible "worst growth" subquotient $Q(\mu)$ called the worst growth piece of $V \otimes F_{-\mu}$. Further, $(Q(\mu) \hat{)}$ contains an irreducible finitedimensional subquotient. Indeed, $L(\gamma-\mu)$ is a subquotient of $(Q(\mu))$.

(3.29) LEMMA. In the setting of (3.28), $Q(\mu)$ is finite dimensional.

Proof. Pick $\omega_{0} \in W\left(\mathfrak{g}, \mathfrak{h}_{m}\right)$ such that $\omega_{0} \gamma$ is $\Phi^{+}\left(\mathfrak{g}, \mathfrak{h}_{m}\right)$-dominant and define translation functors relative to $\Phi^{+}\left(\omega_{0} \gamma\right)$. Place $\Theta_{G}(V) \stackrel{\text { def }}{=} \Theta_{G}\left(\omega_{0} \gamma\right)$ in a coherent family. Observe

(3.30) $Q(\mu)$ is a constituent of $\omega \cdot \Theta_{G}\left(\omega_{0} \gamma\right)$, some $\omega \in W\left(\mathfrak{g}, \mathfrak{h}_{m}\right)$. The KazhdanLusztig conjectures enter in establishing:

(3.31) The asymptotic module of an irreducible constituent of $\omega \cdot \Theta_{G}\left(\omega_{0} \gamma\right)(\omega \in$ $\left.W\left(\mathfrak{g}, \mathfrak{h}_{m}\right)\right)$ is completely reducible.

Assume (3.31) has been verified. Combining (3.31), (3.30), (3.28)(*) and Casselman's result [26, (4.5)]: $Q(\mu)^{\prime}$ has a nondegenerate pairing with $L(\gamma-\mu)$, hence the lemma.

To verify (3.31) recall $[30,7.3 .19]$ that if $\omega=s_{\alpha}$ is a simple reflection,

$$
s_{\alpha} \cdot \Theta_{G}(V)= \begin{cases}-\Theta_{G}(V) & \text { if } \alpha \in \tau(V), \\ \Theta_{G}(V)+\Theta_{G}\left(U_{\alpha} V\right) & \text { if } \alpha \notin \tau(V) .\end{cases}
$$

Assume $\alpha \notin \tau(V)$ and $X_{1}$ is an irreducible constituent of $s_{\alpha} \cdot \Theta_{G}(V)$. We may assume $U_{\alpha} V=X_{1} \oplus \cdots \oplus X_{n}$, by (3.19). Clearly, $\left(U_{\alpha} V\right) \hat{=} \hat{X}_{1} \oplus \cdots \oplus \hat{X}_{n}$. On the other hand, (3.21) shows $\left(U_{\alpha} V\right)^{\hat{n}}$ is completely reducible; hence $\hat{X}_{1}$ is completely reducible. In general, induct on length of $\omega$. Q.E.D.

(3.33) Corollary. Let $V$ be a UEP-module for $G$ and place $V$ in a coherent family $\mathscr{F}$. Then the finite-dimensional irreducible G-modules are among the irreducible constituents of $\mathscr{F}$.

Proof. Using the Jantzen-Zuckerman translation principle [30, 7.3.3] and (3.20):

(3.34) Translation functors map UEP-modules to UEP-modules inside the dominant chamber.

Our proposition is now a consequence of (3.34) and (3.29). Q.E.D.

(3.35) REMARKS. (i) The proof of (3.29) shows: irreducible constituents of successive wall crossings of a UEP-module have completely reducible asymptotic modules. This is the best one can say. For example, let $\pi$ be a discrete series for $S O(4,1)$ and $\alpha \notin \tau(\pi)$. As follows from the Schmid character identity [23,(5.22)], $U_{\alpha} \pi$ is an irreducible representation. By (A.1.4), $U_{\alpha} \pi$ does not have UEP. In fact, character considerations (§4) allow one to show $\left(U_{\alpha} \pi\right)=L_{1} \oplus L_{2}$ with $L_{i}$ irreducible. 
(ii) Lemma (3.29) and its consequence (3.36) are the most delicate points in the paper. In proving (3.29), we utilized the Kazhdan-Lusztig conjectures for HarishChandra modules and lowest weight modules. We remark that a slight modification in (3.28) allows one to prove (3.29) (and (3.38)) in the case: When $V$ is an irreducible Harish-Chandra module with regular integral infinitesimal character and $\hat{V}$ is completely reducible.

As in (3.3), write $M_{m}=\Gamma_{M_{m}} \cdot M_{m}^{0}$. If $F_{\mu}$ is a finite-dimensional $G$-module of extremal weight $\mu$, then $e^{\mu}$ extends to $\Gamma_{M_{m}}$ in a unique manner determined solely by $\mu$. This observation, combined with (3.29), gives

(3.36) Corollary. Let $V$ be a UEP-module for $G$ and assume $H_{0}\left(n_{m}, V\right)$ has lowest $\mathfrak{h}_{m}$-weight $\lambda$. Then the $M_{m}$ action on $H_{0}\left(\mathfrak{n}_{m}, V\right)$ is completely determined by $\lambda$.

We shall need the following result concerning discrete series (which is apparently unpublished, but fairly well known).

(3.37) Proposition. Let $\Theta_{G}\left(\Phi^{+}\left(\mathfrak{h}^{c}\right), \gamma\right)$ be a discrete series representation for $G$. If we place $\Theta_{G}\left(\Phi^{+}\left(\mathfrak{h}^{c}\right), \gamma\right)$ in a coherent family $\mathscr{F}$, then irreducible finite-dimensional representations are among the irreducible constituents of $\mathcal{F}_{\text {. }}$.

(3.38) TheOREM. Let $V$ be a $U E P$-module for $G$ and assume $\operatorname{rank} G=\operatorname{rank} K$. Then $V$ is a regular irreducible constituent of a coherent continuation of some discrete series.

This follows immediately from (3.33) and the next proposition. By the phrase “... regular irreducible constituent...", we understand irreducible constituents with regular infinitesimal character.

(3.39) Proposition. Suppose $V$ is an irreducible Harish-Chandra module for $G$ with regular integral infinitesimal character. Place $V$ in a coherent family $\mathscr{F}$ and assume the finite-dimensional modules are among the constituents in $\mathcal{F}$. If $\operatorname{rank} G=\operatorname{rank} K$, then $V$ is an irreducible constituent of a coherent continuation of some discrete series.

Let $H=T \cdot A$ be a $\theta$-stable Cartan subgroup of $G$, with $T=H \cap K, A=$ $\exp \left(\mathfrak{h}_{0} \cap \mathfrak{g}_{0}\right)$ and $M A$ the Langlands decomposition of the centralizer of $A$ in $G$. A regular character of $H$ is an ordered pair $(\Gamma, \gamma)$, with $\Gamma$ an ordinary character of $H$ and $\gamma \in \mathfrak{h}^{*}$ regular with respect to $\Phi(\mathfrak{m}, \mathfrak{t})$. We assume a compatibility: $\left.\gamma\right|_{\mathfrak{t}}$ is pure imaginary, so that it defines a positive root system $\Phi^{+}(\mathrm{m}, \mathrm{t})$. Write $\delta_{\mathfrak{m}}, \delta_{\mathfrak{m} \cap t}$ to denote the obvious half-sums of positive roots. Then we require $d \Gamma=\gamma+\delta_{\mathrm{m}}$ $2 \delta_{\mathrm{m} \cap \mathrm{f}}$. Now, the pair $(\Gamma, \gamma)$ determines a discrete series representation $\pi_{M}(\gamma)$ on $M$ and a character $e^{\nu}=\exp \left(\left.\gamma\right|_{\mathrm{a}}\right)$. Assume $\gamma$ is regular and choose a parabolic $P=M A N$ such that $\nu$ is negative with respect to $P$-in the sense described prior to (3.6). The triple $\left(P, \pi_{M}(\gamma), e^{\nu}\right)$ is a collection of regular Langlands data. Let

$$
\pi(\gamma)=I_{P}^{G}\left(\pi_{M}(\gamma) \otimes e^{\nu}\right)
$$

and denote by $\bar{\pi}(\gamma)$ the unique irreducible submodule. We will call $\pi(\gamma)$ a standard representation. Finally, $\Theta_{G}(\gamma)$ and $\bar{\Theta}_{G}(\gamma)$ denote the respective characters $[27,30]$. Langlands classification ((3.6)) can now be reformulated.

(3.40) Every irreducible Harish-Chandra module with regular infinitesimal character is equivalent to some $\bar{\pi}(\gamma)$. 
Proof of (3.39) (D. Vogan). We define block equivalence of Harish-Chandra modules to be the equivalence relation generated by $X \sim Y \Leftrightarrow \operatorname{Ext}_{(\mathfrak{g}, k)}^{1}(X, Y) \neq 0$ $[30,9.2]$. Equivalence classes under $\sim$ are called blocks.

Using (3.40) we may view $V=\bar{\pi}(\gamma)$ for a regular character $(\Gamma, \gamma)$ on $H$. By assumption there is a finite-dimensional module $F$ among the irreducible constituents of $\omega \cdot \bar{\Theta}(\gamma), \omega \in W\left(\mathfrak{g}, \mathfrak{h}_{m}\right)$. Using (3.37) and the fact rk $G=$ rk $K$, there exists a discrete series representation $\bar{\pi}\left(\gamma^{\prime}\right)$ having $F$ among the irreducible constituents of $\omega \cdot \bar{\Theta}\left(\gamma^{\prime}\right), \omega \in W\left(\mathfrak{g}, \mathfrak{h}_{m}\right)$. By the proof of $[30,9.2 .11]$, blocks are "closed" under taking $U_{\alpha}$ 's. These observations, and the iteration of (3.32), lead us to conclude:

$$
\bar{\pi}(\gamma) \text { is block equivalent to a discrete series } \bar{\pi}\left(\gamma^{\prime}\right) \text {. }
$$

Assume for the moment:

(3.42) There exists a real root $\alpha \in \Phi(\mathfrak{g}, \mathfrak{h})$ which satisfies the parity condition (see $[30,8.3 .11]$ for the definition).

Now, (3.42) says that $\alpha$ corresponds-via a Cayley transform-to a noncompact imaginary root $\tilde{\alpha}$ on a "more compact Cartan $\tilde{\mathfrak{h}}_{\alpha}$ " and $\alpha$ leads to a new regular character $\left(\tilde{\Gamma}_{\alpha}, \tilde{\gamma}_{\alpha}\right)$ on $\tilde{H}_{\alpha}[30,8.3]$. The parity condition insures the Hecht-Schmid character identities invert [30,8.3]. But this will exhibit $\bar{\pi}(\gamma)$ as a constituent of a continuation of an irreducible character (block equivalent to $\bar{\pi}(\gamma)$ ) with "more compact data". Induction in the split dimension of $\mathfrak{h}$ shows that $\bar{\pi}(\gamma)$ is a constituent of a continuation of some discrete series.

It remains to see (3.42). This will use the notion of compactness of a block. For a precise definition, see [30,9.2.12]. We only remark: All elements of the same block have the same compactness. By [30,9.2.12] and the fact $\bar{\pi}(\gamma)$ is block equivalent to a discrete series (3.41), (3.42) must hold. Q.E.D.

4. A character formula. If $W \in \mathcal{O}^{\prime}$ we define the formal character of $W$, denoted $\Theta(W)$, as in [4]. The usual properties of a character theory prevail $[32,10.6]$.

Let $\Phi_{\mathbf{R}}\left(\mathfrak{g}, \mathfrak{h}_{m}\right)$ denote the real roots in $\Phi\left(\mathfrak{g}, \mathfrak{h}_{m}\right)$. By $[10, \S 5]$, every $\alpha \in \Phi_{\mathbf{R}}\left(\mathfrak{g}, \mathfrak{h}_{m}\right)$ exponentiates to a real-valued character on $H_{m}$. Put

$$
H_{m}^{-}=\left\{h \in H_{m}: e^{\alpha}(h)<1 \text { for all } \alpha \in \Phi_{\mathbf{R}}^{+}\left(\mathfrak{g}, \mathfrak{h}_{m}\right)\right\},
$$

where $\Phi_{\mathbf{R}}^{+}\left(\mathfrak{g}, \mathfrak{h}_{m}\right)=\Phi_{\mathbf{R}}\left(\mathfrak{g}, \mathfrak{h}_{m}\right) \cap \Phi^{+}\left(\mathfrak{g}, \mathfrak{h}_{m}\right)$.

(4.2) Theorem. Let $V$ be a Harish-Chandra module for $G$. Then on $H_{m}^{-} \cap G^{\prime}$ we have the formal identity $\Theta_{G}(V)=\Theta(\hat{V})$.

Proof. Recall, $H_{p}(\mathrm{r}, \hat{V})$ may be computed via the complex $\left\{\hat{V} \otimes \Lambda^{*} \mathrm{r}, d_{p}\right\}$. A formal argument shows

$$
\Theta(\hat{V}) \cdot \sum_{p}(-1)^{p} \Theta\left(\Lambda^{p} \mathrm{r}\right)=\sum_{p}(-1)^{p} \Theta\left(H_{p}(\mathrm{r}, \hat{V})\right) .
$$

Using linear algebra,

$$
\sum_{p}(-1)^{p} \Theta_{H_{m}}\left(\Lambda^{p} \mathrm{r}\right)=\operatorname{det}\left(1-\left.\operatorname{Ad}\right|_{\mathrm{r}}\right)
$$


By (1.5) we obtain a Hochschild-Serre spectral sequence

$$
E_{p, q}^{2}=H_{q}\left(\mathrm{r} \cap \mathrm{m}_{m}, H_{p}\left(\mathfrak{n}_{m}, X\right)\right) \Rightarrow H_{p+q}(\mathrm{r}, X),
$$

where $X=V$ or $X=\hat{V}$. For formal reasons,

$$
\sum_{p, q}(-1)^{p+q} \Theta_{H_{m}}\left(H_{q}\left(\mathrm{r} \cap \mathrm{m}_{m}, H_{p}\left(\mathrm{n}_{m}, V\right)\right)\right)=\sum_{p}(-1)^{p} \Theta_{H_{m}}\left(H_{p}(\mathrm{r}, V)\right) .
$$

Thus, on $H_{m}^{-} \cap G^{\prime}$, Osborne's conjecture $[10,(7.22)]$ gives

$$
\begin{aligned}
\Theta_{G}(V) \cdot \sum_{p}(-1)^{p} \Theta_{H_{m}}\left(\Lambda^{p} \mathrm{r}\right) & =\Theta_{(4.4)} \Theta_{G}(V) \cdot \prod_{\alpha \in \Phi^{+}\left(\mathfrak{g}, \mathfrak{h}_{m}\right)}\left(1-e^{\alpha}\right) \\
& =\sum_{p}(-1)^{p} \Theta_{H_{m}}\left(H_{p}(\mathrm{r}, V)\right) \\
& =\sum_{p, q}(-1)^{p+q} \Theta_{H_{m}}\left(H_{q}\left(\mathrm{r} \cap \mathrm{m}_{m}, H_{p}\left(\mathfrak{n}_{m}, V\right)\right)\right) \\
& =\sum_{p, q}(-1)^{p+q} \Theta\left(H_{q}\left(\mathrm{r} \cap \mathrm{m}_{m}, H_{p}\left(\mathfrak{n}_{m}, \hat{V}\right)\right)\right),
\end{aligned}
$$

where the last step uses (1.23). To complete the proof, apply (4.3) and the analog of (4.6) for $\hat{V}$ to (4.8). Q.E.D.

5. The Hermitian symmetric case. In the case when $G / K$ carries a complex structure, (3.38) can be significantly refined.

(5.1) THEOREM. Let $G$ be a connected semisimple real matrix group whose quotient $G / K$ carries a Hermitian symmetric structure. The UEP-modules for $G$ are precisely the regular irreducible constituents of coherent continuations of the holomorphic discrete series.

In view of (2.7) and (2.8), it is enough to establish (5.1) in the case when $G$ is a connected simple real matrix group. Throughout this section we assume such a $G$ has been fixed. The proof of (5.1) is broken into two steps: (i) an existence result (5.15); (ii) an exhaustion result (5.35).

Because of our assumption, there exists a compact Cartan subgroup $H^{c} \subseteq G$. Simplifying the notation of (3.1), we will let $\Phi\left(\mathfrak{h}^{c}\right)=\Phi\left(\mathfrak{g}, \mathfrak{h}^{c}\right)$. The root system $\Phi\left(\mathfrak{h}^{c}\right)$ is a union of two subsets: $\Phi^{c}\left(\mathfrak{h}^{c}\right)$, consisting of compact roots; and $\Phi^{n}\left(\mathfrak{h}^{c}\right)$, consisting of noncompact roots. Since $G / K$ is Hermitian symmetric:

(5.2) There exists a positive system $\psi$ for $\Phi\left(\mathfrak{h}^{c}\right)$ with the property: $\alpha, \beta \in \Phi^{n}\left(\mathfrak{h}^{c}\right)$ $\cap \psi \Rightarrow \alpha+\beta \notin \Phi\left(\mathfrak{h}^{c}\right)$.

In fact, up to the action of $W\left(G, H^{c}\right)$, there are precisely two positive systems, $\psi$ and $\psi^{\prime}$, with property (5.2) ( $G$ is simple!). Henceforth, we can, and do, assume $\psi \cap \Phi^{c}\left(\mathfrak{h}^{c}\right)=\psi^{\prime} \cap \Phi^{c}\left(\mathfrak{h}^{c}\right)$. We call $\psi$ and $\psi^{\prime}$ the holomorphic positive systems. A choice of $\psi$ determines a splitting of $\mathfrak{g}$ in the Cartan decomposition $\mathfrak{g}=\mathfrak{t} \oplus \mathfrak{g}$ : $\mathfrak{S}=\mathfrak{g}_{+} \oplus \mathfrak{I}_{-}$, with $\mathfrak{g}_{+}=\oplus \mathfrak{g}_{\alpha}, \mathfrak{S}_{-}=\oplus \mathfrak{g}_{-\alpha}$ and $\alpha \in \psi \cap \Phi^{n}\left(\mathfrak{h}^{c}\right)$. Both $\mathfrak{g}_{+}$and $\mathfrak{I}_{-}$ are abelian Ad $K$-invariant subalgebras of $g$ complex conjugate to one another. In $G_{\mathrm{C}}$ they exponentiate to abelian subgroups $S_{ \pm}$of $G_{\mathbf{C}}$. The semidirect product $K_{\mathbf{C}} S_{+}$ is a parabolic subgroup of $G_{\mathbf{C}}$ (not defined over $\mathbf{R}$ ) and $G \cap\left(K_{\mathbf{C}} S_{+}\right)=K$. This 
leads to the usual embedding of $G / K$ into its compact dual $G_{\mathbf{C}} / K_{\mathbf{C}} S_{+}$. We have induced an invariant complex structure on $G / K$ : the elements of $\mathfrak{g}_{\alpha}, \alpha \in \psi \cap \Phi^{n}\left(\mathfrak{h}^{c}\right)$, regarded as left invariant complex vector fields on $G / K$, are antiholomorphic. If we interchange $S_{+}$and $S_{-}$, then $G / K$ attains a complex structure determined by the positive system $-\psi \equiv \psi^{\prime} \bmod \left(W\left(G, H^{c}\right)\right)$.

According to Harish-Chandra [6], the discrete series of $G$ is parametrized by lattice points in a fixed "compact chamber $e$ " of $\Phi^{c}\left(\mathfrak{h}^{c}\right)$. Two "subchambers of $e$ " are determined by the positive systems $\psi$ and $\psi^{\prime}$, which index the "holomorphic discrete series". To be more precise, let $\gamma \in i\left(\mathfrak{h}_{0}^{c}\right)^{*}$ with

$$
\gamma+\delta \in \Lambda_{\mathrm{ch}} \quad \text { and } \quad(\gamma \mid \alpha)>0 \quad \text { for all } \alpha \in \psi \cap \Phi^{c}\left(\mathfrak{h}^{c}\right)
$$

where $\Lambda_{\mathrm{ch}}$ is as in (3.1). Since $\Lambda_{\mathrm{ch}}$ contains the root lattice,

$$
\mu=\gamma-\delta_{c}+\delta_{n}
$$

also lies in $\Lambda_{\text {ch }} ; \delta_{c}$ (resp. $\delta_{n}$ ) denotes the half-sum of roots in $\psi \cap \Phi^{c}\left(\mathfrak{h}^{c}\right)$ (resp. $\left.\psi \cap \Phi^{n}\left(\mathfrak{h}^{c}\right)\right)$. Since $(\mu \mid \alpha) \geqslant 0$ for all $\alpha \in \psi \cap \Phi^{c}\left(\mathfrak{h}^{c}\right)$, there exists an irreducible holomorphic $K_{\mathrm{C}}$-module $V_{\mu}$ of highest weight $\mu$. Let $\omega_{0}=$ opposition involution for $\Phi^{c}\left(\mathfrak{h}^{c}\right) \cap \psi$. Then $V_{\mu} \cong V_{\omega_{0} \mu}=$ the irreducible $K_{\mathbf{C}^{-}}$-module of lowest weight $\omega_{0} \mu$. Extend $V_{\omega_{0} \mu}$ to $K_{\mathrm{C}} S_{+}$using the trivial action on $S_{+}$. By a standard construction $V_{\omega_{0} \mu}$ associates a holomorphic vector bundle to the principal bundle $G_{\mathbf{C}} \rightarrow G_{\mathbf{C}} / K_{\mathbf{C}} S_{+}$, which we denote by $\mathfrak{T}_{\omega_{0} \mu} \rightarrow G_{\mathbf{C}} / K_{\mathbf{C}} S_{+}$. Since $G / K \hookrightarrow G_{\mathbf{C}} / K_{\mathbf{C}} S_{+}$,

$$
\tau_{\omega_{0} \mu} \rightarrow G / K
$$

is a $G$-homogeneous holomorphic vector bundle. Let $H_{\gamma}$ be the space of holomorphic sections of (5.5). The actions of $G$ on $G / K$ and $\mathcal{V}_{\omega_{0} \mu}$ induce a representation $\pi(\psi, \gamma)$ on the $K$-finite vectors in $H_{\gamma}$. A similar discussion leads to $\pi\left(\psi^{\prime}, \gamma^{\prime}\right)$.

(5.6) THEOREM (HARISH-ChANDRA [8]). The representations $\pi(\psi, \gamma)$ are HarishChandra modules for $G$ with infinitesimal character. As $K$-modules, $\pi(\psi, \gamma)=V_{\omega_{0} \mu} \otimes$ (symmetric algebra of $\mathfrak{g}_{+}$). If $(\gamma \mid \alpha)>0$ for all $\alpha \in \psi$, then $\pi(\psi, \gamma)$ globalizes to a discrete series representation of $G$.

Of course, an analog of (5.6) holds for $\pi\left(\psi^{\prime}, \gamma^{\prime}\right)$. The characters of $\pi(\psi, \gamma)$ and $\pi\left(\psi^{\prime}, \gamma^{\prime}\right)$ will be denoted $\Theta_{G}(\psi, \gamma)$ and $\Theta_{G}\left(\psi^{\prime}, \gamma^{\prime}\right)$, respectively. We define the holomorphic discrete series to consist of

$$
\{\pi(\psi, \gamma): \gamma \text { is strictly } \psi \text {-dominant }\} \cup\left\{\pi\left(\psi^{\prime}, \gamma^{\prime}\right): \gamma^{\prime} \text { is strictly } \psi^{\prime} \text {-dominant }\right\} \text {. }
$$

Let $s_{m} \subseteq \Phi^{n}\left(\mathfrak{h}^{c}\right) \cap \psi$ be a maximal strongly orthogonal subset; cf. [19, (2.11)]. We may associate to $s_{m}$ a Cayley transform $c_{s_{m}}$ which is assumed to satisfy

$$
c_{s_{m}}: \mathfrak{h}^{c} \rightarrow \mathfrak{h}_{m} \text { with } c_{s_{m}}(\psi)=\Phi^{+}\left(\mathfrak{g}, \mathfrak{h}_{m}\right) .
$$

Similarly, we have $c_{s_{m}^{\prime}}$ with $c_{s_{m}^{\prime}}\left(\psi^{\prime}\right)=\Phi^{+}\left(\mathfrak{g}, \mathfrak{h}_{m}\right)$. The roots of $c_{s_{m}}\left(s_{m}\right)$ are real roots, hence exponentiate to real-valued characters on $H_{m}[10, \S 5]$. Define

$$
\tilde{H}_{m}=\left\{h \in H_{m}:\left|e^{c_{s_{m}} \beta}(h)\right|<1 \text { for all } \beta \in s_{m}\right\} .
$$

Comparing (4.1) and (5.8), we see

$$
\tilde{H}_{m} \subseteq H_{m}^{-}
$$


(5.10) Theorem (Hecht-MaRTens [8]). The character $\Theta_{G}(\psi, \gamma)$ restricted to the subset (5.8) and pulled back to the corresponding subset of $\left(H^{c}\right)_{\mathbf{C}}$ via $c_{s_{m}}$ is given by

$$
(-1)^{q} \frac{\sum_{\omega \in W\left(G, H^{c}\right)} \varepsilon(\omega) e^{\omega \gamma}}{\prod_{\alpha \in \psi}\left(e^{\alpha / 2}-e^{-\alpha / 2}\right)} ;
$$

here $\varepsilon(\omega)=$ sign of $\omega, q=\frac{1}{2} \operatorname{dim}_{\mathbf{R}} G / K$.

Fix the representations $\pi(\psi, \gamma)$ and $\pi\left(\psi^{\prime}, \gamma^{\prime}\right)$ along with the data required to define them. Since $\psi \cap \Phi^{c}\left(\mathfrak{h}^{c}\right)=\psi^{\prime} \cap \Phi^{c}\left(\mathfrak{h}^{c}\right),-\psi \cap \Phi^{c}\left(\mathfrak{h}^{c}\right)$ and $-\psi^{\prime} \cap \Phi^{c}\left(\mathfrak{h}^{c}\right)$ determine the same Borel subalgebra $\mathfrak{b}_{c}^{-}=\mathfrak{h}^{c} \oplus \tilde{\mathfrak{n}} \subseteq \mathfrak{f}$. The positive systems $-\psi$ and $-\psi^{\prime}$ determine extensions of $\mathfrak{b}_{c}^{-}$to Borel subalgebras of $\mathfrak{g}$;

$$
\mathfrak{b}_{\psi}^{-}=\mathfrak{h}^{c} \oplus \tilde{\mathfrak{n}} \oplus \mathfrak{S}_{-} \quad \text { and } \quad \mathfrak{b}_{\psi^{\prime}}^{-}=\mathfrak{h}^{c} \oplus \tilde{\mathfrak{n}} \oplus \mathfrak{I}_{+},
$$

respectively. Define:

(5.12) $\theta_{\psi}^{\prime}$ (resp. $\left.\theta_{\psi^{\prime}}^{\prime}\right)$ is the category of all finitely generated $\mathfrak{U}(g)$-modules which are $\mathfrak{b}_{\psi}^{-}\left(\right.$resp. $\left.\mathfrak{b}_{\psi^{\prime}}^{-}\right)$-finite.

By $(5.6) \pi(\psi, \gamma)$ is $K$-isomorphic to $V_{\omega_{0} \mu} \otimes S\left(\mathfrak{I}_{+}\right)$; recall (5.4). Using PoincaréBirkhoff-Witt and the fact $\mathfrak{I}_{+}$is abelian,

$$
\mathfrak{U}(\mathfrak{g}) \otimes_{\mathfrak{u}\left(\mathfrak{b}_{\bar{\psi}}^{-}\right)} \mathbf{C}_{\omega_{0} \mu}=\mathfrak{U}(\mathfrak{g}) \otimes_{\mathfrak{U}\left(\mathfrak{f} \oplus \mathfrak{S}_{-}\right)} \mathfrak{U}(\mathfrak{f}) \otimes_{\mathfrak{u}\left(\mathfrak{b}_{c}^{-}\right)} \mathbf{C}_{\omega_{0} \mu} \rightarrow S\left(\mathfrak{g}_{+}\right) \otimes V_{\omega_{0} \mu} \rightarrow 0 .
$$

This shows $\pi(\psi, \gamma)$ is a $\mathfrak{f}^{-}$-quotient of a $\mathfrak{b}_{\psi}^{-}$-Verma module. Similarly, $\pi\left(\psi^{\prime}, \gamma^{\prime}\right)$ is a $\mathfrak{f}$-quotient of a $\mathfrak{b}_{\psi^{\prime}}^{-}$-Verma module. (In fact, arguing as in $(5.13), \pi\left(\psi^{\prime}, \gamma^{\prime}\right)$ is a $\mathfrak{f}$-quotient of a $\boldsymbol{\theta}\left(\mathfrak{b}_{\psi}^{-}\right)$-Verma module, $\theta\left(\mathfrak{b}_{\psi}^{-}\right)$being the Borel subalgebra opposite $\mathfrak{b}_{\psi}^{-}$; Observe $\mathfrak{b}_{\psi^{\prime}}^{-}$and $\theta\left(\mathfrak{b}_{\psi}^{-}\right)$only differ in "compact directions".) Conclude, $\pi(\psi, \gamma)$ (resp. $\left.\pi\left(\psi^{\prime}, \gamma^{\prime}\right)\right)$ decomposes into $\mathfrak{h}^{c}$-weight spaces with finite multiplicities and

$$
\pi(\psi, \gamma)\left(\operatorname{resp} . \pi\left(\psi^{\prime}, \gamma^{\prime}\right)\right) \text { is in } \vartheta_{\psi}^{\prime}\left(\operatorname{resp} . \theta_{\psi^{\prime}}^{\prime}\right) \text {. }
$$

(5.15) Proposition. The regular irreducible constituents of coherent continuations of holomorphic discrete series are UEP-modules.

Proof. We establish (5.15) for the coherent family $\left\{\Theta_{G}(\psi, \gamma+\nu)\right\}_{\nu \in \Lambda_{\mathrm{ch}}}$, the proof for $\psi^{\prime}$ proceeding similarly. Since $\psi$ is holomorphic,

$$
\text { \{compact simple } \psi \text {-roots }\} \equiv\left\{\text { simple }\left(\Phi^{c}\left(\mathfrak{h}^{c}\right) \cap \psi\right) \text {-roots }\right\} \text {. }
$$

In view of this, $[9,(3.6)]$ shows

$$
\Theta_{G}(\psi, \omega \gamma)=\varepsilon(\omega) \Theta_{G}(\psi, \gamma) \text { for all } \omega \in W\left(G, H^{c}\right) \text {. }
$$

The essential properties of coherent families based on $H_{m}$ carry over to coherent families based on $H^{c}$; cf. (3.25). This observation and (5.17) reduce our problem to showing: the irreducible constituents of $\pi(\psi, \gamma)$ are UEP-modules.

By (5.14) the formal character $\Theta(\pi(\psi, \gamma))$ is well defined. Using (5.6) and Weyl's character formula:

$$
\begin{aligned}
\Theta(\pi(\psi, \gamma)) & =\Theta\left(S\left(\mathfrak{S}_{+}\right) \otimes V_{\omega_{0} \mu}\right)=\Theta\left(V_{\omega_{0} \mu}\right) \Theta\left(S\left(\mathfrak{I}_{+}\right)\right) \\
& =\left(\frac{\sum_{\omega \in W\left(\mathfrak{f}, \mathfrak{h}^{c}\right)} \varepsilon(\omega) e^{\omega\left(\mu+\delta_{c}\right)}}{\prod_{\alpha \in \psi \cap \Phi^{c}\left(\mathfrak{h}^{c}\right)}\left(e^{\alpha / 2}-e^{-\alpha / 2}\right)}\right) \cdot\left(\prod_{\alpha \in \psi \cap \Phi^{n}\left(\mathfrak{h}^{c}\right)}\left(1-e^{\alpha}\right)\right)^{-1} .
\end{aligned}
$$


Observe, $W\left(\mathfrak{f}, \mathfrak{h}^{c}\right)=W\left(G, H^{c}\right)$ and the denominator of the second term becomes

$$
(-1)^{q} \cdot e^{\delta_{n}} \cdot \prod_{\alpha \in \psi \cap \Phi^{n}\left(\mathfrak{h}^{c}\right)}\left(e^{\alpha / 2}-e^{-\alpha / 2}\right),
$$

with $q=\frac{1}{2} \operatorname{dim}_{\mathbf{R}} G / K$. Since $\omega\left(\delta_{n}\right)=\delta_{n}$ for all $\omega \in W\left(G, H^{c}\right)$, (5.4) will force $\omega\left(\mu+\delta_{c}\right)-\delta_{n}=\omega\left(\mu+\delta_{c}-\delta_{n}\right)=\omega \gamma$. Combine (5.18), (5.19), (5.10), (5.9) and (4.2) to conclude

$$
\Theta(\pi(\psi, \gamma))=\left.\Theta_{G}(\psi, \gamma)\right|_{\tilde{H}_{m} \cap G^{\prime}}=\theta\left((\pi(\psi, \gamma))^{\wedge}\right)
$$

This exhibits an "equivalence" of the object $\pi(\psi, \gamma) \in \mathcal{O}_{\psi}^{\prime}$ with the object

$$
(\pi(\psi, \gamma)) \hat{)} \in \mathcal{O}^{\prime} \text {. }
$$

We conclude that $(\pi(\psi, \gamma))_{s s} \cong((\pi(\psi, \gamma)))_{s s}$. The exactness of $(\cdots),(1.23)$ and (1.25) finish the proof. Q.E.D.

The proof of (5.1) is complete once we show the representations in (5.15) exhaust the UEP-modules for $G$. The classification shows that $g_{0}$ is among

$$
\mathfrak{s} \mathfrak{u}(p, q), \mathfrak{s}_{\mathfrak{O}} *(2 n), \mathfrak{s}_{\mathfrak{O}}(2, q), \mathfrak{s p}(n, \mathbf{R}), \mathrm{e}_{6(-14)}, \mathrm{e}_{7(-25)} .
$$

For notational simplicity we write $e_{6}=e_{6(-14)}$ and $e_{7}=e_{7(-25)}$.

A somewhat contrived technical device is needed, which we now proceed to describe. The $U E P$-lattice $\mathbf{L}$ (for $G$ ) is defined by

$$
\mathbf{L}=\left\{\lambda \in \mathfrak{h}_{m}^{*}: \text { for some UEP-module } V(\text { for } G),\right.
$$

$$
\left.\lambda \text { is the lowest } \mathfrak{h}_{m} \text {-weight of } H_{0}\left(\mathfrak{n}_{m}, V\right)\right\} \text {. }
$$

Recalling (2.20):

(5.22)(a) We say $\mathbf{L}$ is degenerate if there exists a unique subset $B_{1} \subseteq B$ with the properties: (i) $\left\langle B_{1}\right\rangle=\Phi^{c}\left(\mathfrak{h}^{c}\right)$ (as root systems); (ii) $(\mathbf{L} \mid \alpha) \leqslant 0$ for all $\alpha \in B_{1}$; and (iii) $B \backslash B_{1}$ is long (when two root lengths occur).

(b) We say $\mathbf{L}$ is faithful if there exists a unique pair of subsets $B_{1}, B_{2} \subseteq B$ such that: (i) $\left\langle B_{1}\right\rangle=\left\langle B_{2}\right\rangle=\Phi^{c}\left(\mathfrak{h}^{c}\right)$ (as root systems); (ii) for every $\lambda \in \mathbf{L}$, either $\left(\lambda \mid B_{1}\right) \leqslant 0$ or $\left(\lambda \mid B_{2}\right) \leqslant 0$.

It follows from (5.16) that $\left|B \backslash B_{1}\right|=\left|B \backslash B_{2}\right|=1$. For example, let $G=\operatorname{SP}(2, \mathbf{R})$ (resp. $\mathrm{SU}(2,1)$ ), with $\alpha$ the short simple root (resp. $\beta$ and $\eta$ the simple roots.) We will eventually see ((5.35)): $\mathbf{L}$ is degenerate (resp. faithful) and $\mathbf{L}$ consists of integral $\lambda$ such that: (i) $(\lambda \mid \alpha) \leqslant 0$ (resp. $(\lambda \mid \beta) \leqslant 0$ or $(\lambda \mid \eta) \leqslant 0)$; (ii) $\lambda-\rho$ is regular.

Now our strategy is as follows: We show every lattice $\mathbf{L}$ is degenerate or faithful ((5.29)). In the degenerate (resp. faithful) case we attach to each $\lambda \in \mathbf{L}$ one or two (resp. one) UEP-modules of the type in (5.15). We will then employ Langlands classification to establish exhaustion.

(5.23) Discussion. Let $s \subseteq \Phi^{n}\left(\mathfrak{h}^{c}\right) \cap \psi$ be a strongly orthogonal subset and associate a Cayley transform [19] $c_{s}: \mathfrak{h}^{c} \rightarrow \mathfrak{h}_{s}$, mapping $\mathfrak{h}^{c}$ onto a $\boldsymbol{\theta}$-stable Cartan $\mathfrak{h}_{s}$. There is a splitting $\mathfrak{h}_{s}=\mathfrak{h}_{s,+} \oplus \mathfrak{h}_{s,-}$ into \pm 1 -eigenspaces of $\theta$. The corresponding Cartan subgroup $H_{s}$ has a decomposition $H_{s}=H_{s,+} \cdot H_{s,-}$. The centralizer of $\mathfrak{h}_{s,-}$ in $\mathfrak{g}$ is the Levi factor $\mathfrak{m}_{s} \oplus \mathfrak{a}_{s}\left(\mathfrak{a}_{s}=\mathfrak{h}_{s,-}\right)$ of a cuspidal parabolic subalgebra $\mathfrak{p}_{s}=\mathfrak{m}_{s} \oplus$ $\mathfrak{a}_{s} \oplus \mathfrak{n}_{s}$ and subgroup $P_{s}=M_{s} A_{s} N_{s}$. According to [19, (2.58)] (recall (3.2)):

$$
M_{s} / M_{s}^{+}=\left\{\omega \in W\left(G, H_{s}\right): \omega \mid \mathfrak{h}_{s,-}=1\right\} / W\left(M_{s}^{0}, H_{s,+}^{0}\right) .
$$


Moreover, using the results in $[19, \S 2]$ (most notably $(2.36)$ and $(2.61)),(*)$ is easily computed for specific examples. Finally, up to conjugacy, every cuspidal parabolic subgroup $P$ is of the form $P_{s}$ for some $s$.

Let $\lambda \in \mathbf{L}$. We say $V$ is associated to $\lambda$ (or $\lambda$ corresponds to $V$ ) if $V$ is a UEP-module for $G$ with $H_{0}\left(\mathfrak{n}_{m}, V\right)$ having lowest $\mathfrak{h}_{m}$-weight $\lambda$.

(5.24) Lemma. Let $\lambda \in \mathbf{L}$ correspond to some UEP-module $V$ for $G$. Let $P=M A N$ be a parabolic subgroup ((1.11)) and suppose either (i) or (ii) below holds:

(i) $[\mathfrak{m}, \mathfrak{m}]_{0}=\mathfrak{g l}(3, \mathbf{R}) \oplus($ compact ideal $)$ or $[\mathfrak{m}, \mathfrak{m}]_{0}=\mathfrak{g} \mathrm{o}(2 n+1,1) \oplus$ (compact ideal ).

(ii) $[\mathrm{m}, \mathfrak{m}]_{0}=\mathfrak{g} \mathfrak{o}(2 n, 1) \oplus$ (compact ideal $)$ and $M \neq M^{+}$.

Then $(\lambda \mid \alpha) \leqslant 0$ for all $\alpha \in \Phi^{+}\left(m \oplus a, \mathfrak{h}_{m}\right)$.

Proof. Assume (ii) holds and let $Q$ be an irreducible Harish-Chandra $M A$ quotient of $H_{0}(\mathrm{n}, V)$. By (2.3) and (1.20), $Q$ is a UEP-module for $M A$ and $H_{0}\left(\mathrm{n}_{m} \cap \mathrm{m}, Q\right)$ is $\mathrm{m}_{m} \oplus \mathrm{a}_{m}$-irreducible. According to [16,(3.5)], $\left.Q\right|_{M_{\mathrm{der}}^{0}}$ is a (finite) direct sum of irreducible Harish-Chandra modules for $M_{\mathrm{der}}^{0}$; recall (2.5). Arguing as in the proof of (3.5), we conclude $Q$ restricts to a UEP-module for $M_{\mathrm{der}}^{0}$. Now (A.1.4) shows $\left.Q\right|_{M_{\mathrm{der}}^{0}}$ is finite dimensional or a discrete series. The second possibility cannot occur by (3.5): $M \neq\left. M^{+} \Rightarrow Q\right|_{M}$ is not a discrete series. Conclude $Q$ is finite dimensional, hence (5.24) follows as in the proof of (3.10). Under assumption (i) the proof is similar using (3.8) and (A.1.4). Q.E.D.

(5.25) Lemma. Let $\mathfrak{g}_{0}=\mathfrak{S p}(n, \mathbf{R})$ or $\mathfrak{g}_{0}=\mathfrak{S} \mathfrak{o}(2,2 n+1)$. Then $\mathbf{L}$ is degenerate.

Proof. First, suppose $\mathfrak{g}_{0}=\mathfrak{g} \mathfrak{p}(n, \mathbf{R})$. The classification data (3.12) for this split group are totally expressed by the Satake diagram:

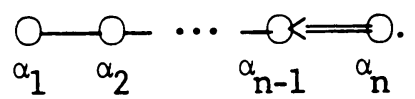

If $n \geqslant 3$, form the sets $R_{i}=\left\{\alpha_{i}, \alpha_{i+1}\right\}$ for $1 \leqslant i \leqslant n-2$. Let $\mathfrak{p}_{i}=\mathfrak{m}_{i} \oplus \mathfrak{a}_{i} \oplus \mathfrak{n}_{i}$ be the associated second from minimal parabolics; recall the proof of (3.13). Notice, $\left[\mathfrak{m}_{i}, \mathfrak{m}_{i}\right]_{0}=\mathfrak{I l}(3, \mathbf{R})$ and so $(5.24)$ forces $(\mathbf{L} \mid \alpha) \leqslant 0$ for $\alpha \in B_{1}=\left\{\alpha_{1}, \ldots, \alpha_{n-1}\right\}$. Since $\mathfrak{f}=\mathfrak{u}(n),\left\langle B_{1}\right\rangle=\Phi(\mathfrak{u}(n))=\Phi^{c}\left(\mathfrak{h}^{c}\right)$. Conclude, $\mathbf{L}$ is degenerate. According to (5.23), every cuspidal parabolic subgroup of $\mathfrak{g}_{0}=\mathfrak{s} \mathfrak{p}(n, \mathbf{R})$ is of the type $\mathfrak{p}_{s}=\mathfrak{m}_{s} \oplus$ $a_{s} \oplus n_{s}$. Using the ideas in (5.23), one shows that the following is true:

(5.26) Let $\mathfrak{g}_{0}=\mathfrak{g} \mathfrak{p}(n, \mathbf{R}), s \subseteq \Phi^{n}\left(\mathfrak{h}^{c}\right) \cap \psi$ a strongly orthogonal set and $\mathfrak{p}_{s}$ the cuspidal parabolic of $(5.23) ; \mathfrak{p}_{m} \subseteq \mathfrak{p}_{s} \subseteq \mathfrak{g} \mathfrak{p}(n, \mathbf{R})$. Then

$$
\mathfrak{m}_{s}=\mathfrak{g} \mathfrak{p}(n-r-2 t, \mathbf{R}) \oplus \mathfrak{I} \mathfrak{l}(2, \mathbf{R}) \oplus \cdots \oplus \mathfrak{I} \mathfrak{l}(2, \mathbf{R}),
$$

where $r=\#$ long roots in $s$, and $t=\#$ short roots in $s=\#$ of copies of $\mathfrak{I} \mathfrak{I}(2, \mathbf{R})$. Furthermore, $\left|M_{s} / M_{s}^{+}\right|=2^{t}$.

In the special case of $n=2$, the Satake diagram is given by

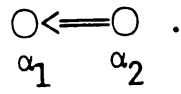


Applying (5.26): there are two kinds of cuspidal parabolics, $p_{1}=m_{1} \oplus a_{1} \oplus \mathfrak{n}_{1}$ and $\mathfrak{p}_{2}=\mathfrak{m}_{2} \oplus \mathfrak{a}_{2} \oplus \mathfrak{n}_{2}$, corresponding to a short and long root, respectively; with $\left|M_{1} / M_{1}^{+}\right|=2,\left|M_{2} / M_{2}^{+}\right|=1$. In both cases, $\left[\mathfrak{m}_{i}, \mathfrak{m}_{i}\right]_{0}=\mathfrak{g l}(2, \mathbf{R})$ and $\mathfrak{g} \mathfrak{l}(2, \mathbf{R}) \cong$ $\mathfrak{g} \mathrm{o}(2,1)$. By $(5.24)\left(\lambda \mid \alpha_{1}\right) \leqslant 0$. If $B_{1}=\left\{\alpha_{1}\right\}$ then the degeneracy of $\mathbf{L}$ follows.

It remains to consider $\mathfrak{g}_{0}=\mathfrak{s} \mathfrak{D}(2,2 n+1), n \geqslant 2$. We let $\left\{e_{1}, \ldots, e_{n+1}\right\}$ be an orthonormal basis for $\left(i \mathfrak{h}_{0}^{c}\right)^{*}$ and $\psi=\psi_{0}, \Phi\left(\mathfrak{h}^{c}\right), \Phi^{c}\left(\mathfrak{h}^{c}\right), \Phi^{n}\left(\mathfrak{h}^{c}\right)$ as in [19, (6.2)]. Then $e_{1}$ becomes a noncompact simple root. Put $s=\left\{e_{1}\right\}$ and form $P_{s}=M_{s} A_{s} N_{s}$. Arguing via (5.23), $M_{s} \neq M_{s}^{+}$. The Satake diagram of $\mathfrak{g}_{0}$ is

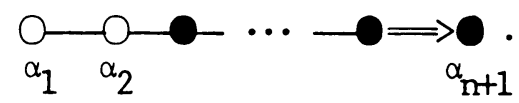

We may identify $B_{1}=\left\{\alpha_{2}, \ldots, \alpha_{n+1}\right\}$ with the simple roots for $\Phi\left(\mathfrak{m}_{s}, \mathfrak{h}_{s,+}\right)$ and $\left[\mathrm{m}_{s}, \mathrm{~m}_{s}\right]_{0}=\mathfrak{g} \mathrm{o}(2 n, 1) \oplus$ (compact ideal). Now, $(5.24)(\mathrm{ii})$ shows $\left(\mathbf{L} \mid B_{1}\right) \leqslant 0$. Since $\left\langle B_{1}\right\rangle=\Phi(\mathfrak{b} \mathfrak{o}(2) \oplus \mathfrak{b} \mathfrak{o}(2 n+1))=\Phi^{c}\left(\mathfrak{h}^{c}\right)$, we have proven $\mathbf{L}$ is degenerate. Q.E.D.

(5.27) LemMA. Fix any $\pi(\psi, \gamma)$ and $\pi\left(\psi^{\prime}, \gamma^{\prime}\right)$. The only possible irreducible subquotient in common is finite dimensional.

Proof. Let $X$ (resp. $Y$ ) be an irreducible subquotient of $\pi(\psi, \gamma)$ (resp. $\left.\pi\left(\psi^{\prime}, \gamma^{\prime}\right)\right)$. Our remarks following (5.13) show: $\pi(\psi, \gamma)$ (resp. $\left.\pi\left(\psi^{\prime}, \gamma^{\prime}\right)\right)$ is a $\mathfrak{k}$-quotient of a $\mathfrak{b}_{\psi}^{-}$-Verma module (resp. $\theta\left(\mathfrak{b}_{\psi}^{-}\right)$-Verma module). This proves that $X$ and $Y$ decompose into $\mathfrak{h}^{c}$-weight spaces with finite multiplicities. Let $\Gamma_{X}$ and $\Gamma_{Y}$ denote the

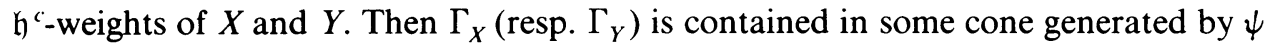
(resp. $-\psi$ ). Since $\psi$ spans $\left(\mathfrak{h}^{c}\right)^{*}, X=Y \Rightarrow \Gamma_{X}=\Gamma_{Y}$ is contained in a region of finite volume. Q.E.D.

(5.28) Lemma. Let $\lambda \in \mathbf{L}$ correspond to a UEP-module $V$ for $G$. Suppose $P=M A N$ is a parabolic subgroup (1.11), with $[\mathrm{m}, \mathrm{m}]_{0}=\mathfrak{S} \mathfrak{u}(n, 1) \oplus$ (compact ideal $)$ for some $n$. Put $B_{\mathrm{m} \oplus \mathfrak{a}}=$ simple $\Phi^{+}\left(\mathrm{m} \oplus \mathfrak{a}, \mathfrak{h}_{m}\right)$ roots and denote by $\alpha_{1}, \alpha_{n}$ the complex roots in $B_{\mathfrak{m} \oplus \mathfrak{a}}$; recall (2.4). Then $\left(\lambda \mid \alpha_{1}\right) \leqslant 0$ or $\left(\lambda \mid \alpha_{n}\right) \leqslant 0$.

Proof. According to (A.2.5), $\mathrm{SU}(n, 1)$ has a faithful UEP-lattice. Conclude: if $Q$ is a UEP-module for $M^{0} A$ and $\lambda$ is the lowest $\mathfrak{h}_{m}$-weight of $H_{0}\left(\mathfrak{n}_{m} \cap \mathrm{m}, Q\right)$, then $\lambda$ is antidominant with respect to one of the complex simple $\Phi^{+}\left(\mathfrak{m} \oplus \mathfrak{a}, \mathfrak{h}_{m}\right)$ roots. Apply (2.3) to finish the proof. Q.E.D.

(5.29) LEMMA. The UEP-lattice for $G$ is degenerate or faithful. More precisely:

(i) $\mathbf{L}$ is degenerate $\Leftrightarrow \mathfrak{g}_{0}=\mathfrak{g} \mathfrak{p}(n, \mathbf{R}), \mathfrak{s} \mathfrak{o}(2, q), \mathfrak{I} \mathfrak{u}(p, p), \mathfrak{S}_{\mathfrak{O}} *(2 n)$ ( $n$ even) or $\mathrm{e}_{7}$.

(ii) $\mathbf{L}$ is faithful $\Leftrightarrow \mathfrak{g}_{0}=\mathfrak{s} \mathfrak{u}(p, q)(p \neq q), \mathfrak{S}^{*} *(2 n)(n$ odd $)$ or $\mathrm{e}_{6}$.

Proof. If $\mathfrak{g}_{0}=\mathfrak{s} \mathfrak{u}(n, 1), \mathfrak{s} \mathfrak{p}(n, \mathbf{R})$ or $\mathfrak{s} \mathfrak{o}(2,2 n+1)$, then apply (A.2.5) and (5.25). We will handle the other possibilities case-by-case. Rather than writing out the full classification data - as in the proof of (3.13) - we only include a Satake diagram, referring to the "proof of (3.13)" when we make explicit claims about the structure of various parabolics. 
In case $\mathfrak{g}_{0}=\mathfrak{S} \mathfrak{o}(2,2 n)(n \geqslant 3)$, the Satake diagram is

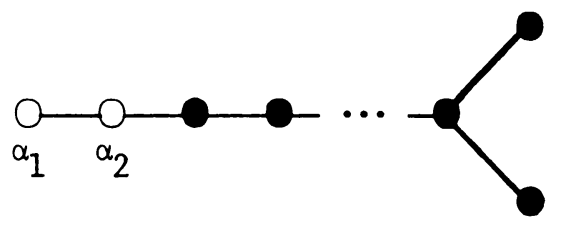

We have two (real) parabolics: $\mathfrak{p}_{1}=\mathfrak{m}_{1} \oplus \mathfrak{a}_{1} \oplus \mathfrak{n}_{1}, \mathfrak{p}_{2}=\mathfrak{m}_{2} \oplus \mathfrak{a}_{2} \oplus \mathfrak{n}_{2}$, with $\left[\mathfrak{m}_{1}, \mathfrak{m}_{1}\right]_{0}=\mathfrak{g l}(2, \mathbf{R}) \oplus$ (compact ideal $), \quad\left[\mathfrak{m}_{2}, \mathfrak{m}_{2}\right]_{0}=\mathfrak{g} \mathrm{o}(2 n-1,1) \oplus$ (compact ideal) and $\alpha_{i} \in \Phi^{+}\left(\mathrm{m}_{i} \oplus \mathrm{a}_{i}, \mathfrak{h}_{m}\right)$ for $i=1,2$. By (5.24) $(\mathbf{L} \mid \alpha) \leqslant 0$ for all $\alpha \in B_{1}=$ $\left\{\alpha_{2}\right\} \cup B_{m}$; recall (2.20). Finally, $\left\langle B_{1}\right\rangle=\Phi(\mathfrak{s} \mathfrak{D}(2) \oplus \mathfrak{s} \mathfrak{D}(2 n))=\Phi^{c}\left(\mathfrak{h}^{c}\right)$.

If $g_{0}=\mathfrak{s} \mathfrak{u}(p, p)$ the Satake diagram is

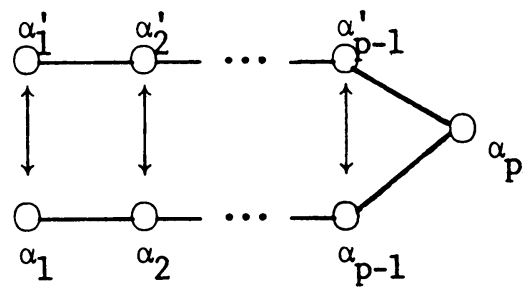

We have (real) parabolics $\mathfrak{p}_{i}=\mathfrak{m}_{i} \oplus \mathrm{a}_{i} \oplus \mathrm{n}_{i}(1 \leqslant i \leqslant p)$, with $\left[\mathfrak{m}_{i}, \mathfrak{m}_{i}\right]_{0}=\mathfrak{g l}(2, \mathbf{C})$ $\oplus$ (compact ideal) for $1 \leqslant i \leqslant p-1$ and $\left[\mathfrak{m}_{p}, \mathfrak{m}_{p}\right]_{0}=\mathfrak{g l}(2, \mathbf{R}) \oplus$ (compact ideal). Further, $\alpha_{i}, \alpha_{i}^{\prime} \in \Phi^{+}\left(\mathfrak{m}_{i} \oplus \mathfrak{a}_{i}, \mathfrak{h}_{m}\right)$ for $1 \leqslant i \leqslant p-1$ and $\alpha_{p} \in \Phi^{+}\left(m_{p} \oplus a_{p}, \mathfrak{h}_{m}\right)$. Recall, $\mathfrak{I} \mathfrak{l}(2, \mathbf{C}) \cong \mathfrak{S} \mathfrak{o}(3,1)$. Apply $(5.24)$ to conclude $(\mathbf{L} \mid \alpha) \leqslant 0$ for $\alpha \in B_{1}=\left\{\alpha_{i}, \alpha_{i}^{\prime}\right.$ : $1 \leqslant i \leqslant p-1\}$. Finally, $\left\langle B_{1}\right\rangle=\Phi(\mathfrak{b} \mathfrak{o}(2) \oplus \mathfrak{I} \mathfrak{u}(p) \oplus \mathfrak{g} \mathfrak{u}(p))=\Phi^{c}\left(\mathfrak{h}^{c}\right)$.

In case $\mathrm{g}_{0}=\mathfrak{S O}^{*}(2 n)$ ( $n$ even), the Satake diagram is

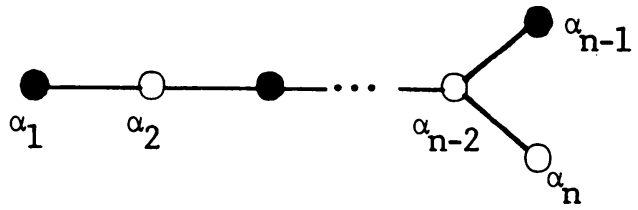

We have (real) parabolics $\mathfrak{p}_{j}=\mathfrak{m}_{j} \oplus \mathfrak{a}_{j} \oplus \mathfrak{n}_{j}(j=2 k, 1 \leqslant k \leqslant n / 2)$, with $\left[\mathfrak{m}_{j}, \mathfrak{m}_{j}\right]_{0}$ $=\mathfrak{g} \mathfrak{o}(5,1) \oplus$ (compact ideal) for $1 \leqslant k<n / 2,\left[\mathfrak{m}_{n}, \mathfrak{m}_{n}\right]_{0}=\mathfrak{s l}(2, \mathbf{R}) \oplus$ (compact ideal) and $\alpha_{j} \in \Phi^{+}\left(\mathfrak{m}_{j} \oplus \mathfrak{a}_{j}, \mathfrak{h}_{m}\right)$. Since $\left(\mathbf{L} \mid B_{m}\right) \leqslant 0$ always holds, $\left(\mathbf{L} \mid B_{1}\right) \leqslant 0$ for $B_{1}=\left\{\alpha_{1}, \ldots, \alpha_{n-1}\right\}$. Finally, $\left\langle B_{1}\right\rangle=\Phi(\mathfrak{u}(n))=\Phi^{c}\left(\mathfrak{h}^{c}\right)$.

The last degenerate possibility is $g_{0}=e_{7}$ which has Satake diagram

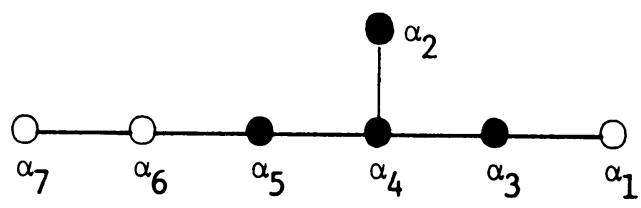


Obtain (real) parabolics $\mathfrak{p}_{i}=\mathfrak{m}_{i} \oplus \mathfrak{a}_{i} \oplus \mathfrak{n}_{i}(i=1,6,7)$, with $\left[\mathfrak{m}_{i}, \mathfrak{m}_{i}\right]=\mathfrak{g} \mathfrak{o}(9,1) \oplus$ (compact ideal) for $i=1,6$ and $\left[\mathrm{m}_{7}, \mathrm{~m}_{7}\right]_{0}=\mathfrak{g} \mathfrak{l}(2, \mathbf{R}) \oplus$ (compact ideal). Further, $\alpha_{i} \in \Phi^{+}\left(\mathfrak{m}_{i} \oplus \mathfrak{a}_{i}, \mathfrak{h}_{m}\right)(i=1,6,7)$. If $B_{1}=\left\{\alpha_{1}, \ldots, \alpha_{6}\right\}$, then $\left(\mathbf{L} \mid B_{1}\right) \leqslant 0$ via $(5.24)$ and $\left\langle B_{1}\right\rangle=\Phi\left(e_{6} \oplus \mathbf{R}\right)=\Phi^{c}\left(\mathfrak{h}^{c}\right)$.

For $\mathfrak{g}_{0}=\mathfrak{s} \mathfrak{u}(p, q)(2 \leqslant p<q)$, the Satake diagram is

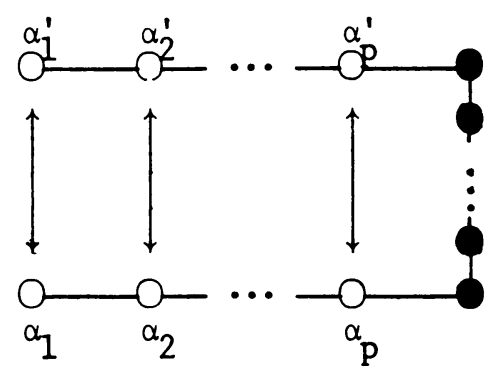

We have (real) parabolics $\mathfrak{p}_{i}=\mathrm{m}_{i} \oplus \mathfrak{a}_{i} \oplus \mathrm{n}_{i}(1 \leqslant i \leqslant p)$, with $\left[\mathrm{m}_{i}, \mathrm{~m}_{i}\right]_{0}=\mathfrak{g} \mathfrak{l}(2, \mathbf{C})$ $\oplus$ (compact ideal) for $1 \leqslant i \leqslant p-1,\left[\mathfrak{m}_{p}, \mathfrak{m}_{p}\right]_{0}=\mathfrak{s} \mathfrak{u}(q-p+1,1) \oplus$ (compact ideal) and $\alpha_{i}, \alpha_{i}^{\prime} \in \Phi^{+}\left(\mathrm{m}_{i} \oplus \mathfrak{a}_{i}, \mathfrak{h}_{m}\right)$ for $1 \leqslant i \leqslant p$. Using (5.28), $\left(\mathbf{L} \mid \alpha_{p}\right) \leqslant 0$ or $\left(\mathbf{L} \mid \alpha_{p}^{\prime}\right) \leqslant 0$. Since $\left(\mathbf{L} \mid B_{m}\right) \leqslant 0$ holds, (5.24) shows $\left(\mathbf{L} \mid B_{1}\right) \leqslant 0$ or $\left(\mathbf{L} \mid B_{2}\right) \leqslant 0$, where $B_{1}=\left\{\alpha_{i}, \alpha_{i}^{\prime}: 1 \leqslant i \leqslant p-1\right\} \cup\left\{\alpha_{p}\right\} \cup B_{m}$ and $B_{2}=\left\{\alpha_{i}, \alpha_{i}^{\prime}: 1 \leqslant i \leqslant p-1\right\} \cup\left\{\alpha_{p}^{\prime}\right\}$ $\cup B_{m}$. Finally, $\left\langle B_{1}\right\rangle=\left\langle B_{2}\right\rangle=\Phi(\mathfrak{g} \mathfrak{u}(p) \oplus \mathfrak{g} \mathfrak{u}(q) \oplus \mathbf{R})=\Phi^{c}\left(\mathfrak{h}^{c}\right)$.

If $\mathfrak{g}_{0}=\mathfrak{B}^{*}(2 n)$ ( $n$ odd), then the Satake diagram is

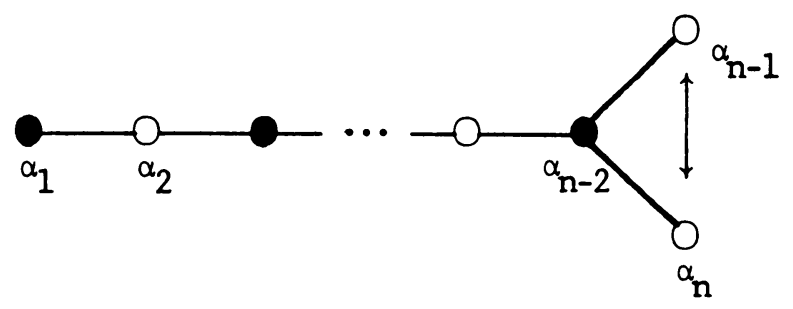

We have (real) parabolics $\mathfrak{p}_{j}=\mathrm{m}_{j} \oplus \mathrm{a}_{j} \oplus \mathfrak{n}_{j}\left(j=2 k, 1 \leqslant k \leqslant \frac{1}{2}(n-1)\right)$, with $\left[\mathfrak{m}_{j}, \mathfrak{m}_{j}\right]_{0}=\mathfrak{s} \mathfrak{o}(5,1) \oplus$ (compact ideal) for $1 \leqslant k \leqslant \frac{1}{2}(n-3)$ and $\left[\mathfrak{m}_{n-1}, \mathfrak{m}_{n-1}\right]_{0}=$ $\mathfrak{S} \mathfrak{u}(3,1) \oplus$ (compact ideal). Further, $\alpha_{j} \in \Phi^{+}\left(\mathfrak{m}_{j} \oplus \mathfrak{a}_{j}, \mathfrak{h}_{m}\right)$ for $1 \leqslant k \leqslant \frac{1}{2}(n-3)$ and $\alpha_{n-1}, \alpha_{n} \in \Phi^{+}\left(m_{n-1} \oplus \mathfrak{a}_{n-1}, \mathfrak{h}_{m}\right)$. Let $B_{1}=\left\{\alpha_{1}, \ldots, \alpha_{n-2}\right\} \cup\left\{\alpha_{n-1}\right\}$ and $B_{2}$ $=\left\{\alpha_{1}, \ldots, \alpha_{n-2}\right\} \cup\left\{\alpha_{n}\right\}$. An application of (5.24) and (5.28) shows $\left(\mathbf{L} \mid B_{1}\right) \leqslant 0$ or $\left(\mathbf{L} \mid B_{2}\right) \leqslant 0$. Finally, $\left\langle B_{1}\right\rangle=\left\langle B_{2}\right\rangle=\Phi(\mathfrak{u}(n))=\Phi^{c}\left(\mathfrak{h}^{c}\right)$.

Our last case is $g_{0}=e_{6}$ which has Satake diagram

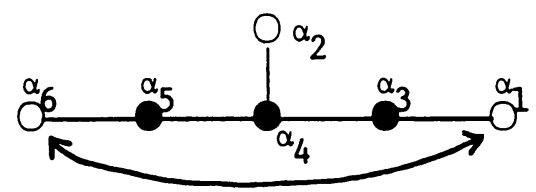


There exists (real) parabolics $\mathfrak{p}_{i}=\mathrm{m}_{i} \oplus \mathfrak{a}_{i} \oplus \mathfrak{n}_{i}(i=1,2)$ which satisfy $\left[\mathrm{m}_{1}, \mathrm{~m}_{1}\right]_{0}=$ $\mathfrak{s} \mathfrak{u}(5,1) \oplus$ (compact ideal) and $\left[\mathrm{m}_{2}, \mathrm{~m}_{2}\right]_{0}=\mathfrak{s} \mathfrak{o}(7,1) \oplus$ (compact ideal). Further, $\alpha_{2} \in \Phi^{+}\left(\mathfrak{m}_{2} \oplus \mathfrak{a}_{2}, \mathfrak{h}_{m}\right) ; \alpha_{1}, \alpha_{6} \in \Phi^{+}\left(\mathfrak{m}_{1} \oplus \mathfrak{a}_{1}, \mathfrak{h}_{m}\right)$. Let $B_{1}=\left\{\alpha_{1}, \ldots, \alpha_{5}\right\}$ and $B_{2}$ $=\left\{\alpha_{2}, \ldots, \alpha_{6}\right\}$. Then (5.24) and (5.28) show $\left(\mathbf{L} \mid B_{1}\right) \leqslant 0$ or $\left(\mathbf{L} \mid B_{2}\right) \leqslant 0$. Finally, $\left\langle B_{1}\right\rangle=\left\langle B_{2}\right\rangle=\Phi(\mathfrak{s} \mathfrak{o}(10) \oplus \mathbf{R})=\Phi^{c}\left(\mathfrak{h}^{c}\right)$. Q.E.D.

(5.30) Proposition. Suppose $V$ is a UEP-module for $G$. If $V$ is a discrete series, then $V$ is a holomorphic discrete series.

Note. Using Knapp and Wallach's "Szegö embeddings of discrete series" [13], Silva [22,(16.1)] established (5.30) for the real rank one case. Our proof will use iterated Schmid embeddings and the translation principle.

Proof. Let $\psi=\psi_{1}, \psi_{2}, \ldots, \psi_{m-1}, \psi_{m}=\psi^{\prime}$ be positive systems indexing the discrete series of $G$; recall (3.1).

(5.31) If $\psi_{1}$ is not a holomorphic positive system, then $\psi_{i}$ contains at least two simple noncompact roots.

(Suppose $\psi_{i}$ has simple roots $\alpha_{1}, \ldots, \alpha_{n}, \beta$, where $\alpha_{1}, \ldots, \alpha_{n}$ are compact and $\beta$ is noncompact. Any noncompact $\psi_{i}$-positive root $\eta$ has the form $\eta=\sum k_{i} \alpha_{i}+l \beta$, $k_{i} \in \mathbf{N}, l>0$. Since $G / K$ is Hermitian symmetric, $\mathfrak{t}$ has one-dimensional center. Thus, $\left\{\alpha_{1}, \alpha_{2}, \ldots, \alpha_{n}\right\}=\left\{\right.$ simple $\left(\psi_{i} \cap \Phi^{c}\left(\mathfrak{h}^{c}\right)\right)$-roots $\}$. If $\tau$ is a compact root, then $\tau=\Sigma l_{i} \alpha_{i}, l_{i} \in \mathbf{Z}$. If $\eta+\tau$ is a root, we conclude that $\eta+\tau=\Sigma\left(k_{i}+l_{i}\right) \alpha_{i}+l \beta$ is $\psi_{i}$-positive since $l>0$. Now $\left[\mathfrak{f}, \mathfrak{S}_{+}\left(\psi_{i}\right)\right] \subseteq \mathfrak{S}_{+}\left(\psi_{i}\right)$, where $\mathfrak{S}_{+}\left(\psi_{i}\right)$ is the positive part of $\mathfrak{s}$ determined by $\psi_{i}$. But this condition is equivalent to $\psi_{i}$ being holomorphic.)

Let $V$ be a UEP-discrete series corresponding to $\psi_{i}$. Argue by contradiction, assuming $\psi_{i}$ is not holomorphic. Let $\beta_{1}, \beta_{2}$ be noncompact simple $\psi_{i}$ roots, (5.31). Form the maximal cuspidal parabolic subgroups $P_{\beta_{1}}, P_{\beta_{2}}$ as in (5.23). We may assume (by conjugating) that $P_{m} \subseteq P_{\beta} \subseteq G$ for $j=1,2$. Using Schmid's character identity [21]:

(5.32)(a) $V \hookrightarrow I_{P_{\beta}}^{G}\left(W_{j} \otimes e^{\nu}\right)(j=1,2)$, where $W_{j} \otimes e^{\nu}$, is explicitly constructed in [21]. In particular, $W_{j}$ is a discrete series on $M_{\beta_{j}}$.

We claim:

(b) There exists only one maximal cuspidal parabolic subgroup $P=M A N$, $P_{m} \subseteq P \subseteq G$, such that (5.32)(a) holds.

If $\mathfrak{g}_{0}=\mathfrak{s} \mathfrak{u}(p, q)(1 \leqslant p \leqslant q), \mathfrak{s} \mathrm{o}(2,2 n), \mathfrak{s} \mathrm{o} *(2 n), \mathrm{e}_{6}$ or $\mathrm{e}_{7}$, then the classification data (3.12) establishes (5.32)(b). If $g_{0}=\mathfrak{s} \mathfrak{D}(2,2 n+1)$, the proof of (5.25) shows there exist precisely two maximal cuspidal parabolics $P_{m} \subseteq P_{k} \subseteq G(k=1,2)$, where $\left|M_{1} / M_{1}^{+}\right|=1$ and $\left|M_{2} / M_{2}^{+}\right|=2$. Since $V$ is a UEP-module, only $P_{1}$ is allowed in (5.32)(a); recall (3.5), (2.3). The case of $\mathfrak{g}_{0}=\mathfrak{s p}(n, \mathbf{R})$ is handled similarly, using (5.26). This proves $(5.32)(\mathrm{b})$ and we conclude

(5.33) $V \hookrightarrow I_{P}^{G}\left(W_{j} \otimes e^{\nu_{j}}\right)(j=1,2)$, where $W_{j} \otimes e^{\nu_{\jmath}}$ is as in (5.32)(a) and $P=P_{\beta_{1}}$ $=P_{\beta_{2}}, P_{m} \subseteq P \subseteq G$.

The constructions in [21] make it clear that the embeddings in (5.33) must differ for generic $\psi_{i}$-discrete series. Indeed, the $\nu_{j}$ are generically different. (Recall, translation functors map $V$ to a $\psi_{i}$-discrete series inside the $\psi_{i}$ chamber [34].) The $W_{j}$ in 
(5.33) have Schmid embeddings into cuspidal data for $M$. Iterating, we obtain embeddings

(5.34) $V \hookrightarrow I_{P_{m}}^{G}\left(\tilde{W}_{j} \otimes e^{\tilde{\nu}}\right)(j=1,2)$, where $\tilde{\nu}_{j}$ are different for generic $\psi_{i}$-discrete series.

According to the translation principle (3.34) applied to $V$, all $\psi_{i}$-discrete series have UEP-contradiction. Q.E.D.

(5.35) Proposition. Let $\mathbf{L}^{\prime}$ consist of all $\lambda \in \mathbf{L}$ which are not in the closed negative chamber determined by $\Phi^{+}\left(\mathfrak{g}, \mathfrak{h}_{m}\right)$.

(i) If $\mathbf{L}$ is degenerate and $\lambda \in \mathbf{L}^{\prime}$, then we may associate two distinct UEP-modules $V_{\lambda}$ and $W_{\lambda}$ to $\lambda$, and these are the only UEP-modules associated to $\lambda$. Further, $V_{\lambda}$ (resp. $\left.W_{\lambda}\right)$ is a subquotient of some $\pi(\psi, \gamma)\left(\right.$ resp. $\left.\pi\left(\psi^{\prime}, \gamma^{\prime}\right)\right)$.

(ii) If $\mathbf{L}$ is faithful and $\lambda \in \mathbf{L}^{\prime}$, then we may associate one UEP-module $V_{\lambda}$ to $\lambda$ and this is the only UEP-module associated to $\lambda$. Further, $V_{\lambda}$ is a subquotient of either $\pi(\psi, \gamma)$ or $\pi\left(\psi^{\prime}, \gamma^{\prime}\right)$ for some $\gamma$ or $\gamma^{\prime}$.

Proof. In (5.13) we noted that $\pi(\psi, \gamma)$ is a $\mathfrak{f}$-quotient of a $\mathfrak{b}_{\psi}^{-}$-Verma module of lowest weight $\omega_{0} \mu=\omega_{0} \gamma+\delta$; recall (5.4). Let $Q_{\psi}(\gamma)$ be the unique irreducible subquotient of $\pi(\psi, \gamma)$ which contains a vector of $\mathfrak{h}^{c}$-weight $\omega_{0} \gamma+\delta$. Similarly obtain $Q_{\psi^{\prime}}\left(\gamma^{\prime}\right)$ containing a vector of $\mathfrak{h}^{c}$-weight $\omega_{0} \gamma^{\prime}+\delta^{\prime}$ in $\pi\left(\psi^{\prime}, \gamma^{\prime}\right)$. Define

$$
\Lambda_{\psi}^{-}=\left\{\tau \in\left(\mathfrak{h}^{c}\right)^{*}:(\tau \mid \alpha) \leqslant 0 \text { for } \alpha \in \Phi^{c}\left(\mathfrak{h}^{c}\right) \cap \psi, \tau-\delta \text { is regular, } \tau-\delta \in \Lambda_{\mathrm{ch}}\right\} .
$$

Replacing $\delta$ by $\delta^{\prime}$ and noting $\Phi^{c}\left(\mathfrak{h}^{c}\right) \cap \psi=\Phi^{c}\left(\mathfrak{h}^{c}\right) \cap \psi^{\prime}$, we are led to $\Lambda_{\psi^{\prime}}^{-}$. Observe:

(5.36) Every $\tau \in \Lambda_{\psi}^{-}$(resp. $\Lambda_{\psi^{\prime}}^{-}$) has the form $\tau=\omega_{0} \gamma+\delta$ (resp. $\tau=\omega_{0} \gamma^{\prime}+\delta^{\prime}$ ) for some $\gamma$ (resp. $\gamma^{\prime}$ ) in the positive compact chamber.

By (5.29) $\mathbf{L}$ is faithful or degenerate. Define

$$
\Lambda_{B_{i}}^{-}=\left\{\lambda \in\left(\mathfrak{h}_{m}\right)^{*}:(\lambda \mid \alpha) \leqslant 0 \text { for } \alpha \in B_{i}, \lambda-\rho \text { is regular, } \lambda-\rho \in \Lambda_{m}\right\},
$$

with $B_{1}, B_{2}$ as in (5.22) and $\Lambda_{m}$ as in $\S 3$. If $\mathbf{L}$ is degenerate only $\Lambda_{B_{1}}^{-}$is defined. The choice of $B_{1}$ and $B_{2}$ in (5.22) shows the Cayley transforms $c_{s_{m}}$ and $c_{s^{\prime}{ }_{m}}$ (5.7) must satisfy:

$$
\begin{aligned}
& c_{s_{m}} \text { maps } \Lambda_{\psi}^{-} 1: 1 \text { and onto } \Lambda_{B_{1}}^{-} \text {or } \Lambda_{B_{2}}^{-} \text {, } \\
& c_{s_{m}^{\prime}} \text { maps } \Lambda_{\psi^{\prime}}^{-}, 1: 1 \text { and onto } \Lambda_{B_{1}}^{-} \text {or } \Lambda_{B_{2}}^{-} .
\end{aligned}
$$

Consequently:

(5.38)(i) L degenerate: $\Lambda_{B_{1}}^{-} \subseteq \mathbf{L} \subseteq \Lambda_{B_{1}}^{-}$and

$$
c_{s_{m}}\left(\Lambda_{\psi}^{-}\right)=c_{s_{m}^{\prime}}\left(\Lambda_{\psi^{\prime}}^{-}\right)=\Lambda_{B_{1}}^{-} .
$$

(ii) $\mathbf{L}$ faithful: $c_{s_{n}}\left(\Lambda_{\psi}^{-}\right) \cup c_{s_{m}^{\prime}}\left(\Lambda_{\psi^{\prime}}^{-}\right) \subseteq \mathbf{L} \subseteq \Lambda_{B_{1}}^{-} \cup \Lambda_{B_{2}}^{-}$.

(The first inclusion in (5.38)(i) (resp. (5.38)(ii)) uses (5.20), (5.36) and (5.37). The second inclusions in (5.38)(i), (ii) follow from (5.22), the fact that $\hat{V}=L(\lambda-\dot{\rho})$ for any $V$ associated to $\lambda$ (1.25), and noting $\mathbf{L}-\rho \subseteq \Lambda_{m}$; recall (3.33).) 
Case (1). $\mathbf{L}$ is degenerate.

From (5.38)(i), $\mathbf{L}=\Lambda_{B_{1}}^{-}$and every $\lambda \in \mathbf{L}^{\prime}$ is associated to some $Q_{\psi}(\gamma)$ and some $Q_{\psi^{\prime}}\left(\gamma^{\prime}\right)$. By (5.27) and the definition of $\mathbf{L}^{\prime}$, we have associated two distinct UEPmodules to each $\lambda \in \mathbf{L}^{\prime}$.

It remains to see that there are no other UEP-modules associated to $\lambda$. If $\lambda$ is associated to a discrete series, then (5.30) proves this. Thus, assume all modules associated to our fixed $\lambda \in \mathbf{L}^{\prime}$ are nontempered regular Langlands representations. If $J_{P}^{G}\left(W \otimes e^{\nu}\right)$ is associated to $\lambda$, then:

(5.39)(i) $P=M A N \neq P_{m}$; (ii) the noncompact part of $[\mathrm{m}, \mathrm{m}]_{0}$ has real rank strictly less than that of $\mathrm{g}_{0}$; (iii) the noncompact part of $[\mathrm{m}, \mathrm{m}]_{0}$ is among the

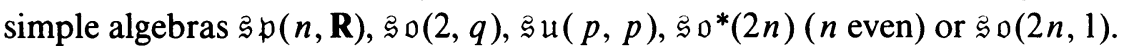

(If $P=P_{m}$, then by (3.36) and (3.3), $W$ is uniquely determined by $\lambda$. We now have two distinct modules associated to $\lambda$ with the same Langlands data - a contradiction to (3.6). The remaining parts of (5.39) follow from (5.29) and the classification, together with (5.26), (2.3) and (3.5) in the case of $g_{0}=\lessgtr p(n, \mathbf{R})$.) Now, (2.3) and (3.5) show $M=M^{+}$. By (3.3) and (3.36), $W$ is uniquely determined by $\lambda$ on the component group of $M$. Arguing by induction in real rank and using (5.29), (5.39) and (A.1.4)(ii) (to get induction going), we see there exist at most two sets of data associated to $\lambda$.

Case (2). $\mathbf{L}$ is faithful.

As argued in Case (1), we may assume $\lambda \in \mathbf{L}^{\prime}$ corresponds to a nontempered Langlands representation. If $J_{P}^{G}\left(W \otimes e^{\nu}\right)$ is associated to $\lambda$, then, using the classification and (5.29):

(5.40)(i) $P=M A N=P_{m}$, or (ii) the noncompact part of $[\mathrm{m}, \mathrm{m}]_{0}$ is of real rank strictly less than that of $\mathfrak{g}_{0}$ and is among the simple algebras $\mathfrak{s} \mathfrak{u}(p, q)(p \neq q)$, $30^{*}(2 n)(n$ odd $)$.

If $P=P_{m}$, then (3.36) and (3.3) show $W$ is uniquely determined by $\lambda$. When $P \neq P_{m}$, (2.3) and (3.5) force $M=M^{+}$. Again, (3.3) and (3.36) show the component representation of $W$ is uniquely determined by $\lambda$. Argue by induction in real rank, using (5.29), (5.40) and (A.2.6) (to get induction going) to see $\left.W\right|_{M^{0}}$ is uniquely determined by $\lambda$. Conclude

(5.41) Each $\lambda \in \mathbf{L}^{\prime}$ corresponds to one and only one UEP-module.

Combine (5.37), (5.38)(ii), (5.27) and (5.41) to see: $c_{s_{m}}\left(\Lambda_{\psi}^{-}\right)=\Lambda_{B_{i}}^{-}, c_{s_{m}^{\prime}}\left(\Lambda_{\psi^{\prime}}^{-}\right)=\Lambda_{B_{1}}^{-}$ $(i \neq j)$ and $\Lambda_{B_{1}}^{-} \cup \Lambda_{B_{2}}^{-} \subseteq \mathbf{L} \subseteq \Lambda_{B_{1}}^{-} \cup \Lambda_{B_{2}}^{-}$. This shows every $\lambda \in \mathbf{L}^{\prime}$ associates to a unique module of the type in (5.15). Q.E.D.

Combining (5.35) and (3.37) we obtain (5.1).

(5.42) REMARK. We have shown: The asymptotic functor $(\cdots)$, restricted to non-finite-dimensional UEP-modules of $G$, is $1: 1$ (resp. 2:1) as $\mathbf{L}$ is faithful (resp. degenerate). This explains our terminology.

6. The split case. We now solve the UEP-problem whenever $G$ is a split group.

(6.1) THEOREM. Let $G$ be a connected simple real split matrix group. If $\mathfrak{g}_{0} \cong \mathfrak{S p}(n, \mathbf{R})$, then the UEP-modules for $G$ are given by (5.1). If $\mathrm{g}_{0} \neq \mathfrak{g} \mathfrak{p}(n, \mathbf{R})$, then the UEP-modules for $G$ are precisely the irreducible finite-dimensional representations. 
By the classification of real forms and the technique of proof used in (3.13), a proof of (6.1) amounts to showing: The UEP-modules for $\mathfrak{s} \mathfrak{o}(n, n+1)(n \geqslant 3)$ and $\mathrm{g}_{2(2)}$ are finite dimensional.

(6.2) LEMMA. The UEP-modules for $\mathfrak{g}_{0}=\mathfrak{s} \mathfrak{o}(n, n+1)(n \geqslant 3)$ are precisely the irreducible finite-dimensional representations.

Proof. The Satake diagram is

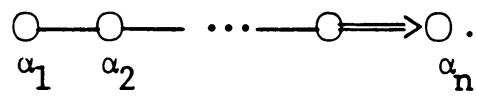

Form real parabolics $\mathfrak{p}=\mathrm{m} \oplus \mathfrak{a} \oplus \mathfrak{n}$ and $\mathfrak{p}^{\prime}=\mathfrak{m}^{\prime} \oplus \mathfrak{a}^{\prime} \oplus \mathfrak{n}^{\prime}$ such that $[\mathrm{m}, \mathrm{m}]_{0}=$ $\mathfrak{s} l(n, \mathbf{R}), \quad\left[\mathfrak{m}^{\prime}, \mathfrak{m}^{\prime}\right]_{0}=\mathfrak{s p}(2, \mathbf{R}), \quad \alpha_{1}, \ldots, \alpha_{n-1} \in \Phi^{+}\left(\mathfrak{m} \oplus \mathfrak{a}, \mathfrak{h}_{m}\right)$ and $\alpha_{n-1}, \quad \alpha_{n} \in$ $\Phi^{+}\left(\mathfrak{m}^{\prime} \oplus \mathfrak{a}^{\prime}, \mathfrak{h}_{m}\right)$. Let $\mathbf{L}$ be defined as in (5.21) with $\lambda \in \mathbf{L}$ and $V$ associated to $\lambda$. By an analog of $(5.24)(i),\left(\lambda \mid \alpha_{i}\right) \leqslant 0$ for $1 \leqslant i \leqslant n-1$. The degeneracy of $\mathfrak{s p}(2, \mathbf{R})$, (5.25) leads to the conclusion $\left(\lambda \mid \alpha_{n}\right) \leqslant 0$. As usual, lowest weight theory, (1.25) and (3.7) show that $V=\hat{V}$ is finite dimensional. Q.E.D.

In order to deal with $\mathfrak{g}_{2(2)}$, we will need some $M^{+}$considerations. Specifically, let $\alpha$ and $\beta$ be the simple short and long roots of $g_{2(2)}$, respectively. Using the remarks in (5.23) (which hold whenever $G$ has discrete series):

(6.3) Up to conjugacy $P_{\beta}$ and $P_{\beta+\alpha}$ are the only maximal cuspidal parabolic subgroups of $G_{2(2)}$. Further, $\left|M_{\beta} / M_{\beta}^{+}\right| \neq 1$ and $\left|M_{\beta+\alpha} / M_{\beta+\alpha}^{+}\right| \neq 1$.

(6.4) LEMMA. The UEP-modules for $G_{2(2)}$ are precisely the irreducible finite-dimensional representations.

Proof. Using (6.3) and an argument as in the proof of (3.8), we need only show: The discrete series for $G_{2(2)}$ are never UEP-modules. Let $\psi$ be the positive system determined by our choice of $\alpha$ and $\beta$ above. Then $\psi$ corresponds to the so-called Borel-de Siebenthal chamber; cf. [19,(9.1)]. Let $\pi(\psi, \gamma)$ be a $\psi$-discrete series and $\Theta_{G}(\psi, \gamma)$ the corresponding character. Vargas showed [25]:

(6.5) The character $\Theta_{G}(\psi, \gamma)$, restricted to the set (5.8) and pulled back to the corresponding subset of $\left(H^{c}\right)_{C}$ via $c_{s_{\eta \eta}}$, is given by

$$
2(-1)^{q} \frac{\sum_{\omega \in W_{u}} \varepsilon(\omega) e^{T \omega \lambda}}{\prod_{\alpha \in \dot{\psi}}\left(e^{\alpha / 2}-e^{-\alpha / 2}\right)},
$$

some $T \in W\left(\mathfrak{g}, \mathfrak{h}^{c}\right), W_{u}=$ Weyl group generated by reflections about compact simple $\psi$-roots, $q=\frac{1}{2} \operatorname{dim}_{\mathbf{R}} G / K$.

Combining (6.5), (5.9) and (4.2) we conclude $(\pi(\psi, \gamma) \hat{)}$ is not irreducible. By $(1.25), \pi(\psi, \gamma)$ is not a UEP-module.

Now, $G_{2(2)}$ contains three kinds of discrete series, corresponding to positive systems: $\psi, s_{\beta} \psi, s_{\beta+\alpha} \cdot s_{\beta} \psi$. Consider a $s_{\beta} \psi$-discrete series $\pi\left(s_{\beta} \psi, \gamma_{1}\right)$ with character $\Theta_{G}\left(s_{\beta} \psi, \gamma_{1}\right)$. By Schmid's character identity [21],

$$
\Theta_{G}\left(s_{\beta} \psi, \gamma_{1}\right)+\Theta_{G}\left(\psi, \gamma_{1}\right)=\Theta_{G}\left(\psi, \beta, \gamma_{1}\right) \text {, }
$$


where $\Theta_{G}\left(\psi, \beta, \gamma_{1}\right)=I_{P_{\beta}}^{G}(\phi), \phi=\phi_{1} \otimes e^{\nu}$,

$$
\phi_{1}= \begin{cases}\sum_{m \in M_{\beta} / M_{\beta}^{+}} \phi_{0} \circ \mathrm{Ad}_{m} & \text { on } M_{\beta}^{+}, \\ 0 & \text { otherwise, }\end{cases}
$$

for some invariant eigendistribution $\phi_{0}$ on $M_{\beta}^{+}$. Every coset of $M_{\beta} / M_{\beta}^{+}$has a representative $m$ normalizing $A_{m}[19,(5.4)]$. Such an $m$, via Ad, operates on $A_{m}$ as an element of $W\left(\mathrm{~g}, \mathrm{a}_{m}\right)$. Hence, because $\phi$ is an IED on $M_{\beta}^{+} A_{\beta},\left.\phi_{1}\right|_{A_{m}}=\left|M_{\beta} / M_{\beta}^{+}\right|$ $\left.\cdot \phi_{0}\right|_{A_{m}}$. Combining these observations with (6.3) and [10, §5],

$$
\left.\Theta_{G}\left(\psi, \beta, \gamma_{1}\right)\right|_{\tilde{A}_{m} \cap G^{\prime}}=2 \cdot\left(\frac{\sum_{\omega \in W\left(\mathfrak{g}, \mathfrak{h}^{c}\right)} a_{\omega} e^{\omega \gamma_{1}}}{\prod_{\alpha \in \psi}\left(e^{\alpha / 2}-e^{-\alpha / 2}\right)}\right),
$$

with $a_{\omega} \in \mathbf{C}$. Place the $\psi$-discrete series character $\Theta_{G}\left(\psi, s_{\beta} \gamma_{1}\right)$ in a (unique) coherent family. As was noted by Schmid, this amounts to "freezing" the coefficients in the expression (3.1), then letting the parameter vary [34, §5]. The properties of coherence will then insure that (6.5) holds for the invariant eigendistribution $\Theta_{G}\left(\psi, \gamma_{1}\right)$. This remark, (6.6) and (6.8) combine to yield

$$
\left.\Theta_{G}\left(s_{\beta} \psi, \gamma_{1}\right)\right|_{\tilde{A}_{m} \cap G^{\prime}}=2 \cdot\left(\frac{\sum_{\omega \in W\left(\mathfrak{g}, \mathfrak{h}^{c}\right)} a_{\omega}^{\prime} e^{\omega \gamma_{1}}}{\prod_{\alpha \in \psi}\left(e^{\alpha / 2}-e^{-\alpha / 2}\right)}\right), \quad a_{\omega}^{\prime} \in \mathbf{C} .
$$

By (5.9) and (4.2), $\left(\pi\left(s_{\beta} \psi, \gamma_{1}\right)\right) \hat{~ i s ~ n o t ~ i r r e d u c i b l e . ~ T h u s ~(1.25) ~ s h o w s ~ t h a t ~ t h e ~}$ $s_{\beta} \psi$-discrete series are never UEP-modules. A similar argument handles the $s_{\beta+\alpha}$. $s_{\beta} \psi$-discrete series. Q.E.D.

7. An example. Beyond (5.1), (6.1) and the Appendices, further refinements of (3.38) require substantial effort. In order to illustrate the complex nature of our problem, we will explicitly compute the UEP-modules for $G=\operatorname{SP}(3,1)$; cf. Table 5.

Throughout this section, $G=\operatorname{SP}(3,1)$. The relevant root systems may be identified as follows:

$$
\begin{aligned}
\Phi\left(\mathfrak{h}^{c}\right) & =\left\{ \pm\left(\varepsilon_{i} \pm \varepsilon_{j}\right), 1 \leqslant i<j \leqslant 4 ; \pm 2 \varepsilon_{i}, 1 \leqslant i \leqslant 4\right\}, \\
\Phi\left(\mathfrak{g}, \mathfrak{h}_{m}\right) & =\left\{ \pm\left(e_{i} \pm e_{j}\right), 1 \leqslant i<j \leqslant 4 ; \pm 2 e_{i}, 1 \leqslant i \leqslant 4\right\}, \\
\Phi\left(\mathfrak{m}_{m} \oplus \mathfrak{a}_{m}, \mathfrak{h}_{m}\right) & =\left\{ \pm\left(e_{1}-e_{2}\right), \pm\left(e_{3} \pm e_{4}\right), \pm 2 e_{3}, \pm 2 e_{4}\right\}, \\
\Phi_{\mathbf{R}}\left(\mathfrak{g}, \mathfrak{h}_{m}\right) & =\left\{ \pm\left(e_{1}+e_{2}\right)\right\} .
\end{aligned}
$$

Choose positive systems $\Phi^{+}\left(\mathfrak{h}^{c}\right), \Phi^{+}\left(\mathfrak{g}, \mathfrak{h}_{m}\right), \Phi^{+}\left(\mathfrak{m}_{m} \oplus \mathfrak{a}_{m}, \mathfrak{h}_{m}\right)$ which correspond to choices of simple roots $\Pi=\left\{\varepsilon_{1}-\varepsilon_{2}, \varepsilon_{2}-\varepsilon_{3}, \varepsilon_{3}-\varepsilon_{4}, 2 \varepsilon_{4}\right\}, B=\left\{e_{1}-e_{2}, e_{2}-\right.$ $\left.e_{3}, e_{3}-e_{4}, 2 e_{4}\right\}$ and $B_{m}=\left\{e_{1}-e_{2}, e_{3}-e_{4}, 2 e_{4}\right\}$, respectively. The next lemma is contained in [15].

Note. In [15] $\bar{\pi}(\gamma)$ is the unique irreducible quotient of $\pi(\gamma)$. Consequently, to convert the regular character data of [15] into data of type (3.40), it is necessary to compose the parameters in [15] with the nontrivial element of $W\left(\mathfrak{g}, \mathfrak{a}_{m}\right)$. On the level of regular characters, this amounts to applying $\theta$ to the nontempered data in [15]. We point out,

$$
\theta:\left\{e_{1} \rightarrow-e_{2}, e_{2} \rightarrow-e_{1}, e_{3} \rightarrow e_{3}, e_{4} \rightarrow e_{4}\right\} .
$$


(7.1) LEMMA. The regular Langlands representations of $G$, with trivial infinitesimal character, are parameterized as follows:

(i) There exist four discrete series $\bar{\pi}\left(\gamma_{i}\right)=\pi\left(\gamma_{i}\right)$ with $\gamma_{i} \in\left(\mathfrak{h}^{c}\right)^{*}$ :

$$
\begin{array}{ll}
\gamma_{0}=3 \varepsilon_{1}+2 \varepsilon_{2}+\varepsilon_{3}+4 \varepsilon_{4}, & \gamma_{2}=4 \varepsilon_{1}+3 \varepsilon_{2}+\varepsilon_{3}+2 \varepsilon_{4}, \\
\gamma_{1}=4 \varepsilon_{1}+2 \varepsilon_{2}+\varepsilon_{3}+3 \varepsilon_{4}, & \gamma_{3}=4 \varepsilon_{1}+3 \varepsilon_{2}+2 \varepsilon_{3}+\varepsilon_{4} .
\end{array}
$$

(ii) There exist twelve nontempered regular Langlands representations $\bar{\pi}\left(\gamma_{i j}\right)$ with $\gamma_{i j} \in\left(\mathfrak{h}_{m}\right)^{*}:$

$$
\begin{array}{ll}
\gamma_{01}=-3 e_{1}-4 e_{2}+2 e_{3}+e_{4}, & \gamma_{23}=-e_{1}-2 e_{2}+4 e_{3}+3 e_{4}, \\
\gamma_{02}=-2 e_{1}-4 e_{2}+3 e_{3}+e_{4}, & \gamma_{05}=2 e_{1}-4 e_{2}+3 e_{3}+e_{4}, \\
\gamma_{03}=-e_{1}-4 e_{2}+3 e_{3}+2 e_{4}, & \gamma_{14}=e_{1}-3 e_{2}+4 e_{3}+2 e_{4}, \\
\gamma_{12}=-2 e_{1}-3 e_{2}+4 e_{3}+e_{4}, & \gamma_{06}=3 e_{1}-4 e_{2}+2 e_{3}+e_{4}, \\
\gamma_{13}=-e_{1}-3 e_{2}+4 e_{3}+2 e_{4}, & \gamma_{15}=2 e_{1}-3 e_{2}+4 e_{3}+e_{4}, \\
\gamma_{04}=e_{1}-4 e_{2}+3 e_{3}+2 e_{4}, & \gamma_{24}=e_{1}-2 e_{2}+4 e_{3}+3 e_{4} .
\end{array}
$$

For convenience write $\bar{\pi}\left(\gamma_{i}\right)=\pi_{i}, \bar{\pi}\left(\gamma_{i j}\right)=\bar{\pi}_{i j}$ and $\pi\left(\gamma_{i j}\right)=\pi_{i j}$. If we define translation functors relative to $\Phi^{+}\left(\gamma_{3}\right)$, then the $\tau$-invariants of the irreducible representations of (7.1) will be subsets of $\Pi$. These are listed in Table 4. Recall, a standard representation $\pi(\gamma)$ is attached to a $\theta$-stable Cartan $\mathfrak{h}=\mathrm{t} \oplus \mathfrak{a}$. We define

$$
\begin{aligned}
l^{I}(\gamma) & =\frac{1}{2}\left|\left\{\alpha \in \Phi^{+}(\gamma): \theta \alpha \notin \Phi^{+}(\gamma)\right\}\right|+\frac{1}{2} \operatorname{dim}_{\mathbf{R}} a_{0} \\
& =\text { length of } \bar{\pi}(\gamma) .
\end{aligned}
$$

Discrete series have length zero, while finite-dimensional modules have maximum length. (Length is a measurement of "asymptotic growth".)

Since (3.19) holds for irreducible Harish-Chandra modules, we may apply D. Vogan's algorithm [27] to compute the composition factors of the standard representations $\pi(\gamma)[27,15]$. This information is summarized in Table 4, all formulas being understood on the level of characters.

The next two results-collectively-allow us to determine the "fine structure" of the decompositions in Table 4.

(7.3) TheOREM (Vogan, [28, (7.2); 27, (3.18)], (3.19)). If $\bar{\pi}(\gamma)$ and $\bar{\pi}\left(\gamma_{1}\right)$ are distinct, then $\operatorname{Ext}_{(\mathfrak{g}, K)}^{1}\left(\bar{\pi}(\gamma), \bar{\pi}\left(\gamma_{1}\right)\right) \cong \operatorname{Ext}_{(\mathfrak{g}, K)}^{1}(\bar{\pi}(\gamma)) \neq 0$ only if $l^{I}(\gamma)$ and $l^{I}\left(\gamma_{1}\right)$ have different parities.

(7.4) Recall. $\operatorname{Ext}_{(\mathfrak{g}, K)}^{1}(X, Y) \neq 0 \Leftrightarrow$ there exists a Harish-Chandra module $E$, not isomorphic to $X \oplus Y$, such that $0 \rightarrow Y \rightarrow E \rightarrow X \rightarrow 0$ is exact [30,(9.2.2)].

Consider a standard representation $\pi\left(\gamma_{i j}\right)$ and let $\bar{\phi}_{i j}$ be the dual of the restriction of an inner automorphism of $\mathfrak{g}$ transforming $\mathfrak{h}^{c}$ into $\mathfrak{h}_{m}$ such that $\bar{\phi}_{i j}\left(\Phi^{+}\left(\gamma_{3}\right)\right)=$ $\Phi^{+}\left(\gamma_{i j}\right)$. Set $\alpha=\bar{\phi}_{i j}(\alpha)$ for $\alpha \in \Phi^{+}\left(\gamma_{3}\right)$.

(7.5) Proposition (Vogan). Fix a standard representation $\pi_{i j}$. Suppose $\alpha \in \Pi$, $\bar{\alpha}=\bar{\phi}_{i j}(\alpha)$ is a simple complex root in $\Phi^{+}\left(\gamma_{i j}\right)$ and $\theta \bar{\alpha} \notin \Phi^{+}\left(\gamma_{i j}\right)$. Then $\alpha$ is not in the $\tau$-invariant of any irreducible quotient of $\pi_{i j}$.

Proof. By [30, 8.2.7], there exists an exact sequence

$$
\phi_{\alpha} \psi_{\alpha} \pi_{i j} \rightarrow \pi_{i j} \rightarrow 0 \text {. }
$$


TABLE 4. Composition factors of $\operatorname{SP}(3,1)$

\begin{tabular}{|c|c|c|}
\hline Decomposition & $\begin{array}{c}\tau \text {-invariant of } \\
\text { Langlands submodule }\end{array}$ & $\begin{array}{l}\text { Length of } \\
\text { Langlands } \\
\text { submodule }\end{array}$ \\
\hline$\pi_{01}=\bar{\pi}_{01}+\bar{\pi}_{02}+\bar{\pi}_{23}$ & $\Pi$ & 6 \\
\hline$\pi_{02}=\bar{\pi}_{02}+\bar{\pi}_{03}+\bar{\pi}_{12}+\bar{\pi}_{13}+\bar{\pi}_{23}$ & $\Pi \backslash\left\{\varepsilon_{2}-\varepsilon_{3}\right\}$ & 5 \\
\hline$\pi_{03}=\bar{\pi}_{03}+\bar{\pi}_{04}+\bar{\pi}_{13}+\bar{\pi}_{14}$ & $\Pi \backslash\left\{\varepsilon_{3}-\varepsilon_{4}\right\}$ & 4 \\
\hline$\pi_{12}=\bar{\pi}_{12}+\bar{\pi}_{13}+\pi_{3}$ & $\Pi \backslash\left\{\varepsilon_{1}-\varepsilon_{2}\right\}$ & 4 \\
\hline$\pi_{04}=\bar{\pi}_{04}+\bar{\pi}_{05}+\bar{\pi}_{14}+\bar{\pi}_{15}$ & $\Pi \backslash\left\{2 \varepsilon_{4}\right\}$ & 3 \\
\hline$\pi_{13}=\bar{\pi}_{13}+\bar{\pi}_{14}+\bar{\pi}_{23}+\bar{\pi}_{24}+\pi_{3}$ & $\Pi \backslash\left\{\varepsilon_{1}-\varepsilon_{2}, \varepsilon_{3}-\varepsilon_{4}\right\}$ & 3 \\
\hline$\pi_{05}=\bar{\pi}_{05}+\bar{\pi}_{06}+\bar{\pi}_{15}+\pi_{1}$ & $\Pi \backslash\left\{\varepsilon_{3}-\varepsilon_{4}\right\}$ & 2 \\
\hline$\pi_{14}=\bar{\pi}_{14}+\bar{\pi}_{15}+\bar{\pi}_{24}+\pi_{2}$ & $\Pi \backslash\left\{\varepsilon_{1}-\varepsilon_{2}, 2 \varepsilon_{4}\right\}$ & 2 \\
\hline$\pi_{23}=\bar{\pi}_{23}+\bar{\pi}_{24}$ & $\Pi \backslash\left\{\varepsilon_{2}-\varepsilon_{3}\right\}$ & 2 \\
\hline$\pi_{06}=\bar{\pi}_{06}+\pi_{0}+\pi_{1}$ & $\Pi \backslash\left\{\varepsilon_{2}-\varepsilon_{3}\right\}$ & 1 \\
\hline$\pi_{15}=\bar{\pi}_{15}+\pi_{1}+\pi_{2}$ & $\Pi \backslash\left\{\varepsilon_{1}-\varepsilon_{2}, \varepsilon_{3}-\varepsilon_{4}\right\}$ & 1 \\
\hline$\pi_{24}=\bar{\pi}_{24}+\pi_{2}+\pi_{3}$ & $\Pi \backslash\left\{\varepsilon_{2}-\varepsilon_{3}, 2 \varepsilon_{4}\right\}$ & 1 \\
\hline$\pi_{0}$ & $\Pi \backslash\left\{\varepsilon_{1}-\varepsilon_{2}\right\}$ & 0 \\
\hline$\pi_{1}$ & $\Pi \backslash\left\{\varepsilon_{1}-\varepsilon_{2}, \varepsilon_{2}-\varepsilon_{3}\right\}$ & 0 \\
\hline$\pi_{2}$ & $\Pi \backslash\left\{\varepsilon_{2}-\varepsilon_{3}, \varepsilon_{3}-\varepsilon_{4}\right\}$ & 0 \\
\hline$\pi_{3}$ & $\Pi \backslash\left\{\varepsilon_{3}-\varepsilon_{4}\right\}$ & 0 \\
\hline
\end{tabular}

Let $X$ be an irreducible quotient of $\pi_{i j}$. Then

$$
0 \neq \operatorname{Hom}_{(\mathrm{g}, K)}\left(\pi_{i j}, X\right) \hookrightarrow \operatorname{Hom}_{(\mathrm{g}, K)}\left(\phi_{\alpha} \psi_{\alpha} \pi_{i j}, X\right) \cong \operatorname{Hom}_{(\mathrm{g}, K)}\left(\pi_{i j}, \phi_{\alpha} \psi_{\alpha} X\right),
$$

using the adjointness of translation functors [27,(3.4)]. Q.E.D.

We are now ready to determine the "fine structure" of $\pi_{i j}$. The meaning of the diagrams in (7.6) is explained in the proof.

(7.6) THEOREM. All irreducible submodules and irreducible quotients of the standard representations $\pi_{i j}$ are indicated below:
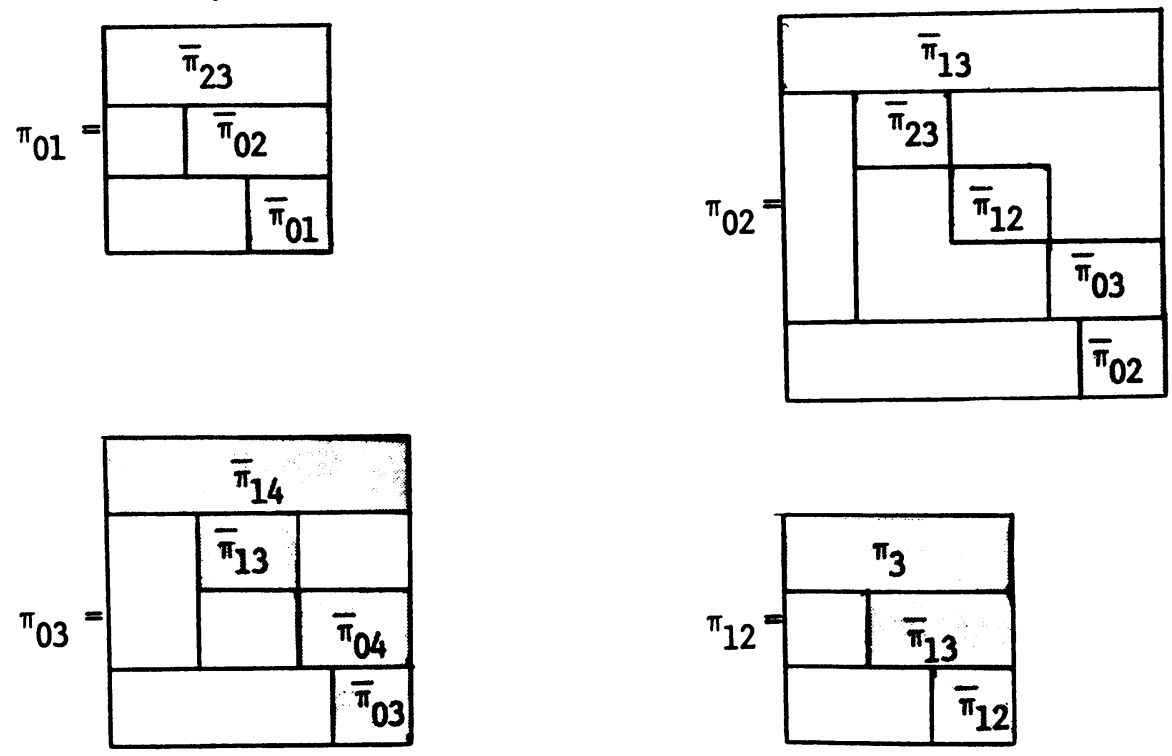

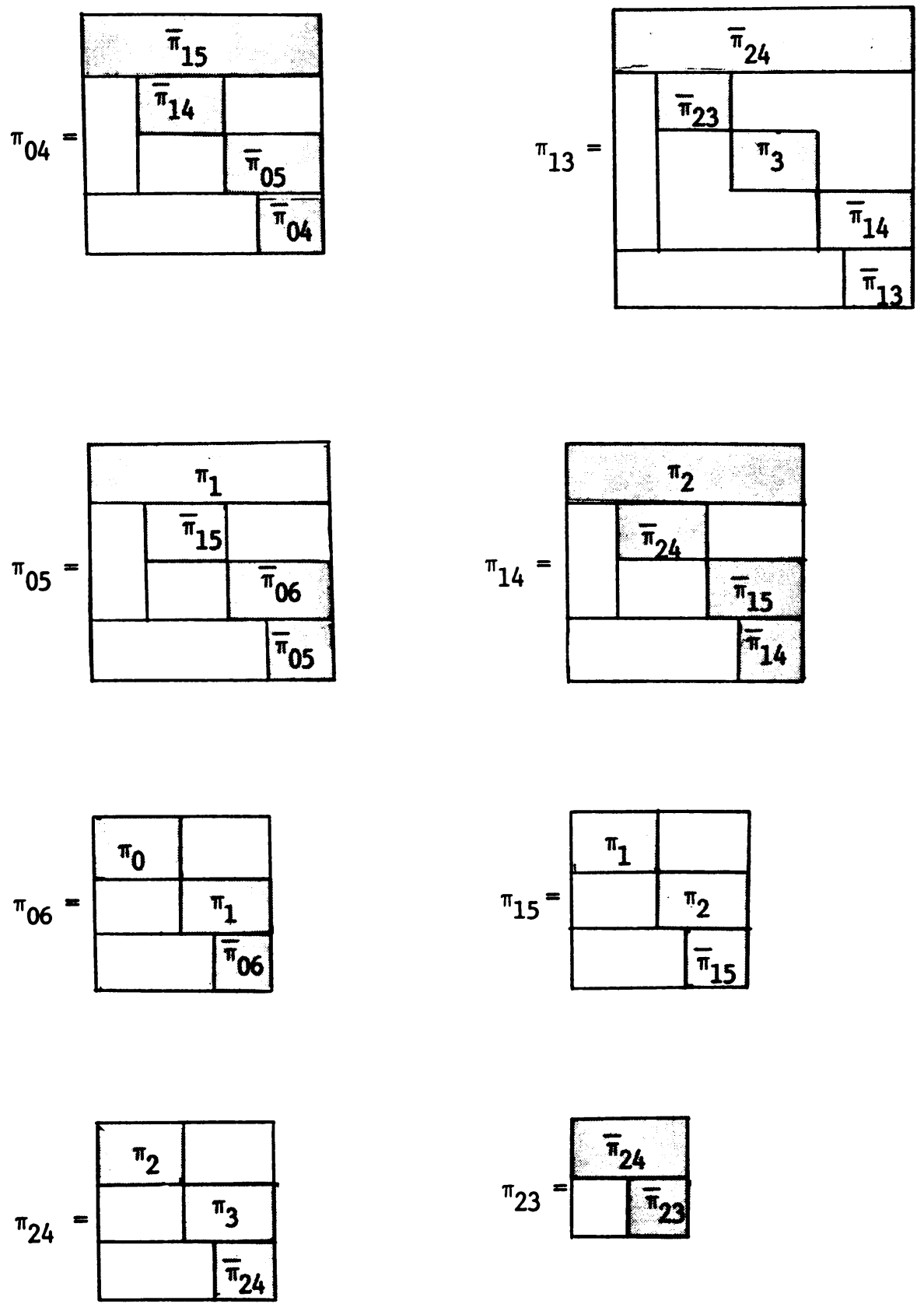

Proof. Submodules (resp. quotients) of $\pi_{i j}$ are on the bottom (resp. top) layer of a box and subquotients are in between. By
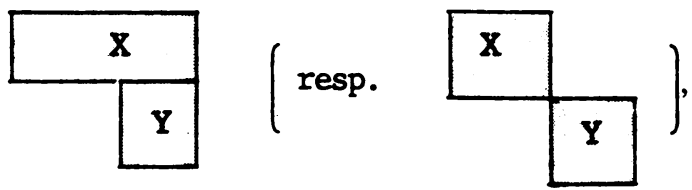
we mean $\operatorname{Ext}_{(\mathfrak{g}, K)}^{1}(X, Y) \neq 0$ (resp. $\left.X \oplus Y\right)$. By (3.6), $\bar{\pi}_{i j}$ is the unique irreducible submodule of $\pi_{i j}$-so the bottom layer of each box is clear. $\pi_{23}$ is obvious (by uniqueness of the subrepresentation $\bar{\pi}_{23}$ ) and $\pi_{06}, \pi_{15}, \pi_{24}$ are Schmid embeddings; cf. $[23,(5.22)]$.

Next, consider $\pi_{01}$ (resp. $\pi_{12}$ ). By (3.6) and Table $4, \bar{\pi}_{01}$ (resp. $\bar{\pi}_{12}$ ) must have nontrivial extension with $\bar{\pi}_{02}$ or $\bar{\pi}_{23}$ (resp. $\bar{\pi}_{13}$ or $\pi_{3}$ ). Table 4 and the parity conditions of (7.3) force $\operatorname{Ext}_{(\mathrm{g}, K)}^{1}\left(\bar{\pi}_{02}, \bar{\pi}_{01}\right) \neq 0$ (resp. $\operatorname{Ext}_{(\mathrm{g}, K)}^{1}\left(\bar{\pi}_{13}, \bar{\pi}_{12}\right) \neq 0$ ). If $\bar{\pi}_{23}$ (resp. $\pi_{3}$ ) has trivial extension with $\bar{\pi}_{02}\left(\right.$ resp. $\left.\bar{\pi}_{13}\right)$, then $\operatorname{Ext}_{(\mathrm{\beta}, K)}^{1}\left(\bar{\pi}_{23}, \bar{\pi}_{01}\right) \neq 0$ (resp. $\left.\operatorname{Ext}_{(\mathrm{g}, K)}^{1}\left(\pi_{3}, \bar{\pi}_{12}\right) \neq 0\right)$-contradiction.

Now consider $\pi_{02}$. The inner automorphism $\bar{\phi}_{02}$ (defined prior to (7.5)) is given by $\theta \circ \phi_{02}$, where [15, p. 457]

$$
\phi_{02}:\left\{\varepsilon_{1} \rightarrow e_{1}, \varepsilon_{2} \rightarrow e_{3}, \varepsilon_{3} \rightarrow e_{2}, \varepsilon_{4} \rightarrow e_{4}\right\}
$$

Let $\alpha=\varepsilon_{1}-\varepsilon_{2}$ and $\beta=\varepsilon_{3}-\varepsilon_{4}$. Then $\bar{\alpha}=-e_{2}-e_{3}$ and $\bar{\beta}=-e_{1}-e_{4}$. Using (7.1): $\left(\theta \bar{\alpha} \mid \gamma_{02}\right)<0,\left(\theta \bar{\beta} \mid \gamma_{02}\right)<0$ and $\bar{\alpha}, \bar{\beta}$ are complex simple $\Phi^{+}\left(\gamma_{02}\right)$ roots. By (7.5), $\alpha, \beta$ are not in the $\tau$-invariant of any irreducible quotient of $\pi_{02}$. In view of Table $4, \bar{\pi}_{13}$ is the only possible irreducible quotient of $\pi_{02}$. By Table 4 the remaining subquotients $\bar{\pi}_{03}, \bar{\pi}_{12}, \bar{\pi}_{23}$ have the same parity. Using (7.3), these subquotients have trivial extensions with one another.

The argument for $\pi_{02}$ works in all other cases. We need the formulas for the various $\bar{\phi}_{i j}=\theta \circ \phi_{i j}$, where $\phi_{i j}$ is given by [15, p. 457]:

$$
\phi_{i j}\left(\varepsilon_{k}\right)= \begin{cases}e_{k+2}, & 1 \leqslant k \leqslant i, \\ e_{1}, & k=i+1, \\ e_{k+1}, & i+2 \leqslant k \leqslant j, \\ e_{2}, & k=j+1, \\ e_{k}, & j+2 \leqslant k \leqslant 4,\end{cases}
$$$$
\phi_{i j}\left(\varepsilon_{k}\right)= \begin{cases}e_{k+2}, & 1 \leqslant k \leqslant i, \\ e_{1}, & k=i+1, \\ e_{k+1}, & i+2 \leqslant k \leqslant 7-j, \\ -e_{2}, & k=8-j, \\ e_{k}, & 9-j \leqslant k \leqslant 4,\end{cases}
$$

Q.E.D.

Note. The above ideas extend to any $G$ of real rank one once analogs of (7.1) and Table 4 are in hand. Our approach, using parity considerations and (7.5), was a consequence of discussions with D. Vogan.

The above results were established under the assumption of trivial infinitesimal character. By the translation principle [30,(8.2.1)], this assumption may be dropped: Theorem (7.6) holds for all standard representations $\pi_{i j}(\chi)$ with a fixed regular integral infinitesimal character $\chi$. We now invoke the fact that $G$ has real rank one. Specifically, (7.6) tells us the "fine structure" of any $I_{P_{m}}^{G}\left(W_{1} \otimes e^{\nu_{1}}\right)$, where $\left(\nu_{1} \mid \alpha\right)<0$ for $\alpha \in \Phi^{+}\left(\mathrm{g}, \mathfrak{a}_{m}\right)$. Dualizing, the structure of $I_{P_{m}}^{G}\left(W_{1} \otimes e^{\nu_{2}}\right)$, where $\left(\nu_{2} \mid \alpha\right)>0$ for $\alpha \in \Phi^{+}\left(g, a_{m}\right)$, is an "upside down" version of some picture in (7.6). In short, the "fine structure" of every $I_{P_{m}}^{G}\left(W \otimes e^{\nu}\right)$ with regular integral infinitesimal character is known. A corollary to these remarks is the following observation:

(7.7) The UEP-modules for $G$ consist of those regular Langlands representations $V$ which satisfy one and only one of the following conditions: (i) $V$ is an irreducible 
submodule in precisely one of the "boxes" in (7.6); (ii) $V$ is an irreducible quotient in precisely one of the "boxes" in (7.6).

Note. A UEP-module $V$ is a nontempered Langlands representation (resp. discrete series) as (i) (resp. (ii)) of (7.7) holds. (This is a consequence of (3.6).)

(7.8) Explanation of Table 5. In Table 5 we have drawn a lattice containing all irreducible representations with a fixed regular integral infinitesimal character. The UEP-modules are circled. Those modules with two (resp. three) embeddings into generalized principal series along $P_{m}$ are enclosed in a box (resp. triangle). The levels of the lattice correspond to length. Placing $\pi_{0}$ in a coherent family $\mathscr{F}_{0}$, the dashed lines connect all irreducible constituents of $\mathscr{F}_{0}$ having the same infinitesimal character as $\pi_{0}$. (This is a computation of successive $U_{\alpha}$ 's and follows using the machinery in [15].) The discrete series $\pi_{0}$ is easily checked to have the Borelde Siebenthal property. Finally, we remark that the coherent family $\mathscr{F}_{1}$, containing $\pi_{1}$, will contain the nontempered UEP-modules among its irreducible constituents. (Again, this is a computation of successive $U_{\alpha}$ 's.)

TABLE 5. Embedding lattice for $\operatorname{SP}(3,1)$

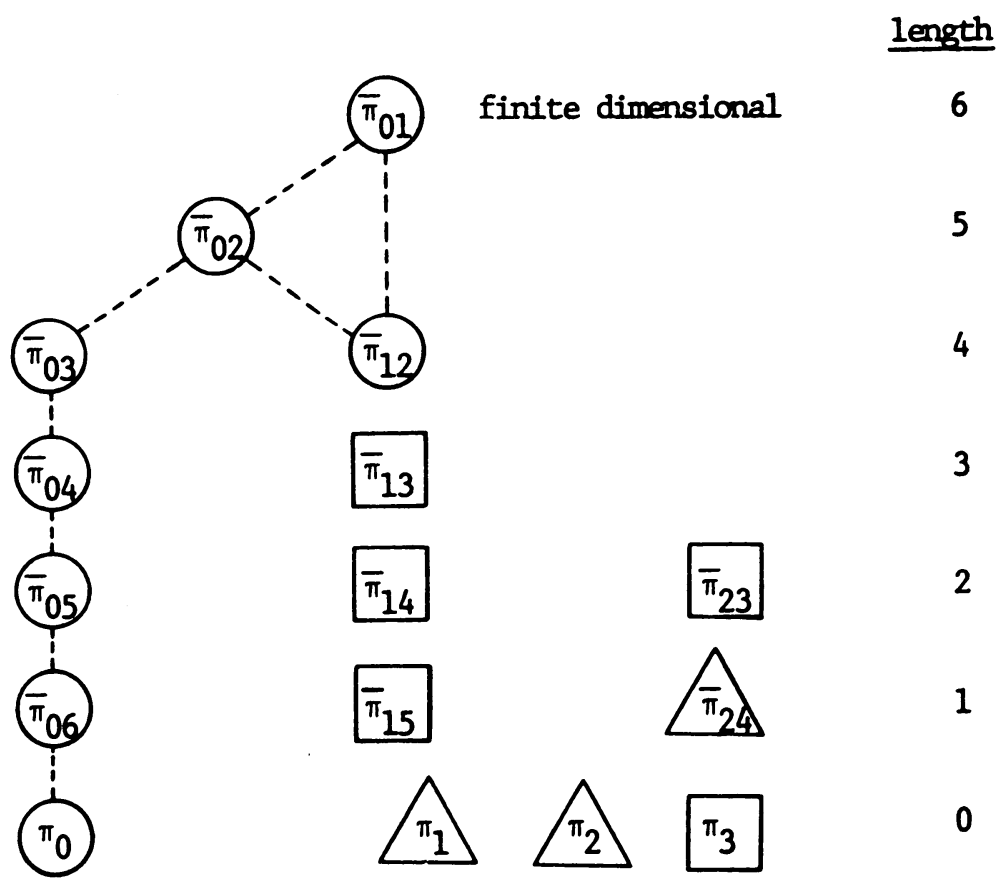

Borel-de Siebenthal

\section{APPENDICES}

A.1. Fine structure of the composition series for $\operatorname{SPIN}(n, 1)$. Throughout this section $G=\operatorname{SPIN}(n, 1)$ with $n \geqslant 2$. This is a connected simple real matrix group embedding into its simply connected complexification. Further, $\mathfrak{I} \mathfrak{l}(2, \mathbf{R}) \cong \mathfrak{g} \mathfrak{o}(2,1)$ and $\mathfrak{I} \mathfrak{I}(2, \mathbf{C}) \cong \mathfrak{S} \mathfrak{o}(3,1)$. (See Table 1 for $\mathfrak{I} \mathfrak{I}(2, \mathbf{R})$ data. $)$ 
TABLE 6. $\mathfrak{S} \mathfrak{O}(n, 1)$

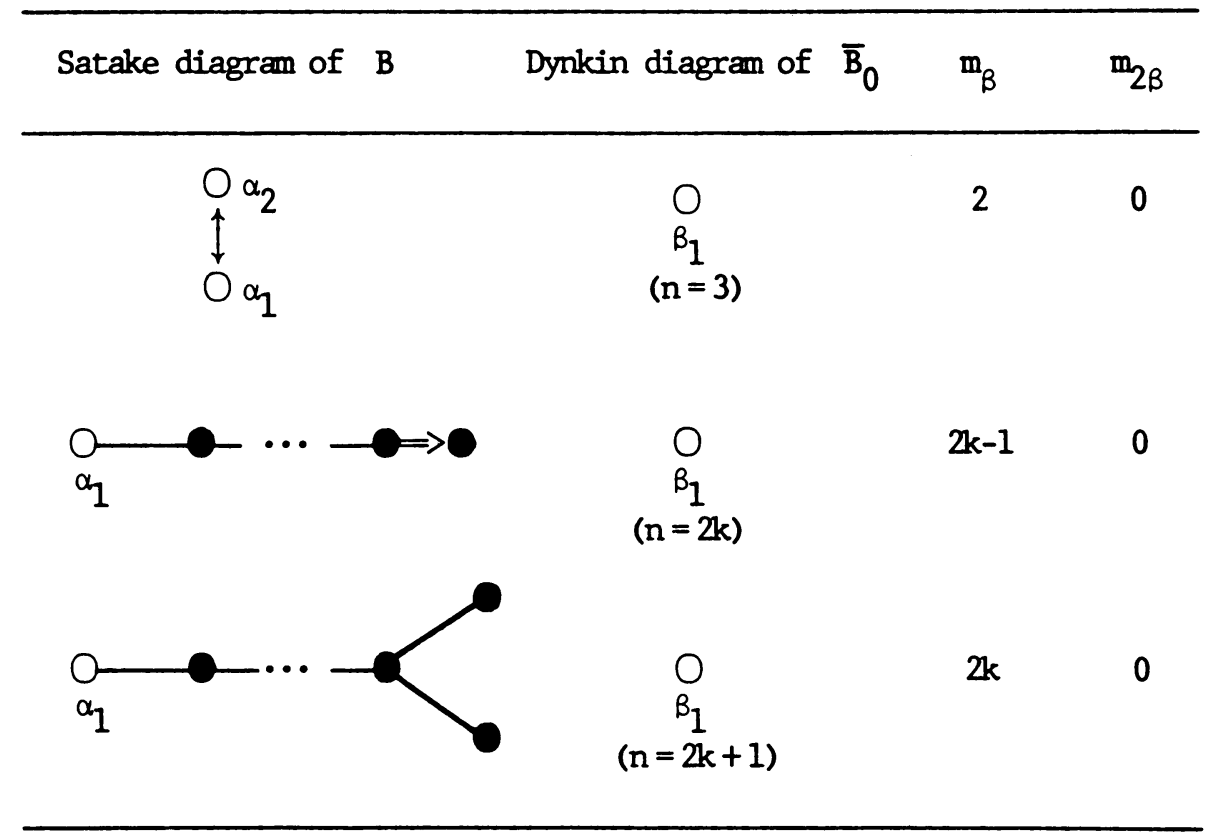

Fix a regular integral infinitesimal character $\chi$. We will describe the "fine structure" of the composition series for each standard representation attached to $\chi$.

(A.1.1) Theorem. If $G=\operatorname{SL}(2, \mathbf{R})$, then the UEP-modules consist of the discrete series and the irreducible finite-dimensional representations.

Proof. As is well known, a standard character attached to $\chi$ is either irreducible or has "fine structure"

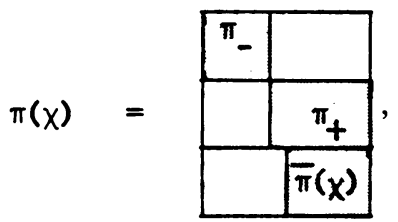

adhering to the conventions in (7.6), where $\pi_{ \pm}$are the discrete series representations attached to $\chi$ and $\bar{\pi}(\chi)$ is finite dimensional. Q.E.D.

The next two results may be found in [33]; also see [18, 24].

(A.1.2) THEOREM. If $G=\operatorname{SPIN}(2 k+1,1)$, there are precisely $k+1$ standard representations attached to $\chi$, say $\pi_{1}, \ldots, \pi_{k+1}$. Furthermore, maintaining the conventions in (7.6), the "fine structure" of the $\pi_{i}$ becomes

$$
\underset{(1 \leq i \leq k)}{\pi_{i}}=\begin{array}{|l|l|}
\bar{\pi}_{i+1} & \text { and } \pi_{k+1}=\bar{\pi}_{k+1} ; \\
\hline & \bar{\pi}_{1}
\end{array}
$$

i.e. $\pi_{k+1}$ is irreducible. 
(A.1.3) Theorem. Let $G=\operatorname{SPIN}(2 k, 1), k \geqslant 2$. There are precisely $k$ standard representations associated to $\chi$, say $\pi_{1}, \ldots, \pi_{k}$. Following the conventions of (7.6), their "fine structure" becomes

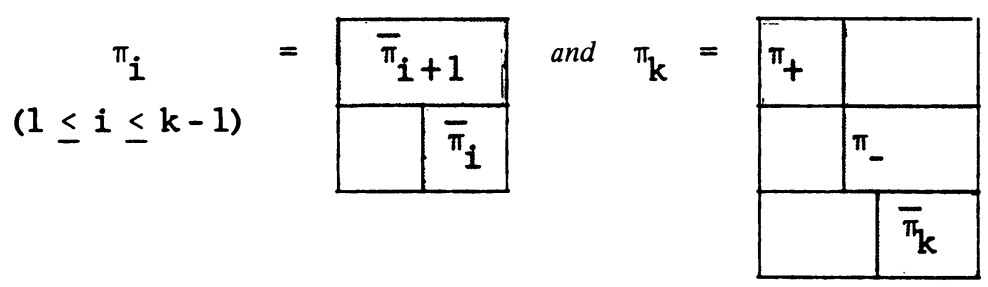

where $\pi_{ \pm}$are the two discret series of $G$ attached to $\chi$.

Arguing as in (7.7), we obtain the following result.

(A.1.4) THEOREM. (i) If $G=\operatorname{SPIN}(2 k+1,1)$, then the UEP-modules are precisely the irreducible finite-dimensional representations.

(ii) If $G=\operatorname{SPIN}(2 k, 1)$, then the UEP-modules are precisely the discrete series and the irreducible finite-dimensional representations. To each tempered $\lambda \in \mathbf{L}((5.21))$ there correspond precisely two UEP-discrete series.

A.2. Fine structure of the composition series for $\mathrm{SU}(n, 1)$. Throughout this section $G=\mathrm{SU}(n, 1)$ with $n \geqslant 2$. This is a connected simple real matrix group embedding into its simply connected complexification.

TABLE 7. $\mathfrak{g} \mathcal{H}(n, 1)$

\begin{tabular}{|c|c|c|c|}
\hline Satake diagram of $B$ & Dynkin diagram of $\bar{B}_{0}$ & $\mathrm{~m}_{B}$ & $\mathrm{~m}_{2 \beta}$ \\
\hline$\underbrace{}_{\alpha_{n}}$ & $\begin{array}{l}\bigcirc \\
B_{1}\end{array}$ & $2(n-1)$ & 1 \\
\hline
\end{tabular}

Fix a regular integral infinitesimal character $\chi$. We will describe the "fine structure" of the composition series for each standard representation attached to $\chi$.

(A.2.1) THEOREM [33]. Let $G=\mathrm{SU}(n, 1)$. The regular Langlands representations attached to $\chi$ may be parametrized as follows:

(i) $\bar{\pi}_{i, j}, 1 \leqslant i \leqslant n, 1 \leqslant j \leqslant n-i+1$ (nontempered).

(ii) $\pi_{1+i, n+1-i}, 0 \leqslant i \leqslant n$ (discrete series).

Zelobenko [33] has given some "fine structure" information for the standard representations $\pi_{i j}$ of (A.2.1). A priori his information is not as sharp as the type in (7.6) but will be adequate for our purposes. We will understand

$\pi_{i j}=$\begin{tabular}{|l|l|}
\hline$A$ & $B$ \\
\hline$C$ & $D$ \\
\hline
\end{tabular}


to mean: $A$ is an irreducible quotient of $\pi_{i j}, D$ is an irreducible submodule of $\pi_{i j}, C$ and $B$ are irreducible subquotients of $\pi_{i j}$. Using (3.6), we know $D=\bar{\pi}_{i j}$ is the unique irreducible submodule of $\pi_{i j}$. However, these pictures do not tell us anything about $\operatorname{Ext}_{(\mathrm{q}, k)}^{1}(B, C)$, nor do we know $A$ is the only quotient. This could be resolved using analysis as in $§ 7$.

Using (A.2.1), (A.2.2) and [33], the structure of the $\pi_{i j}$ are given in Table 8.

TABLE 8. Fine structure of standard modules of $\operatorname{SU}(n, 1)$

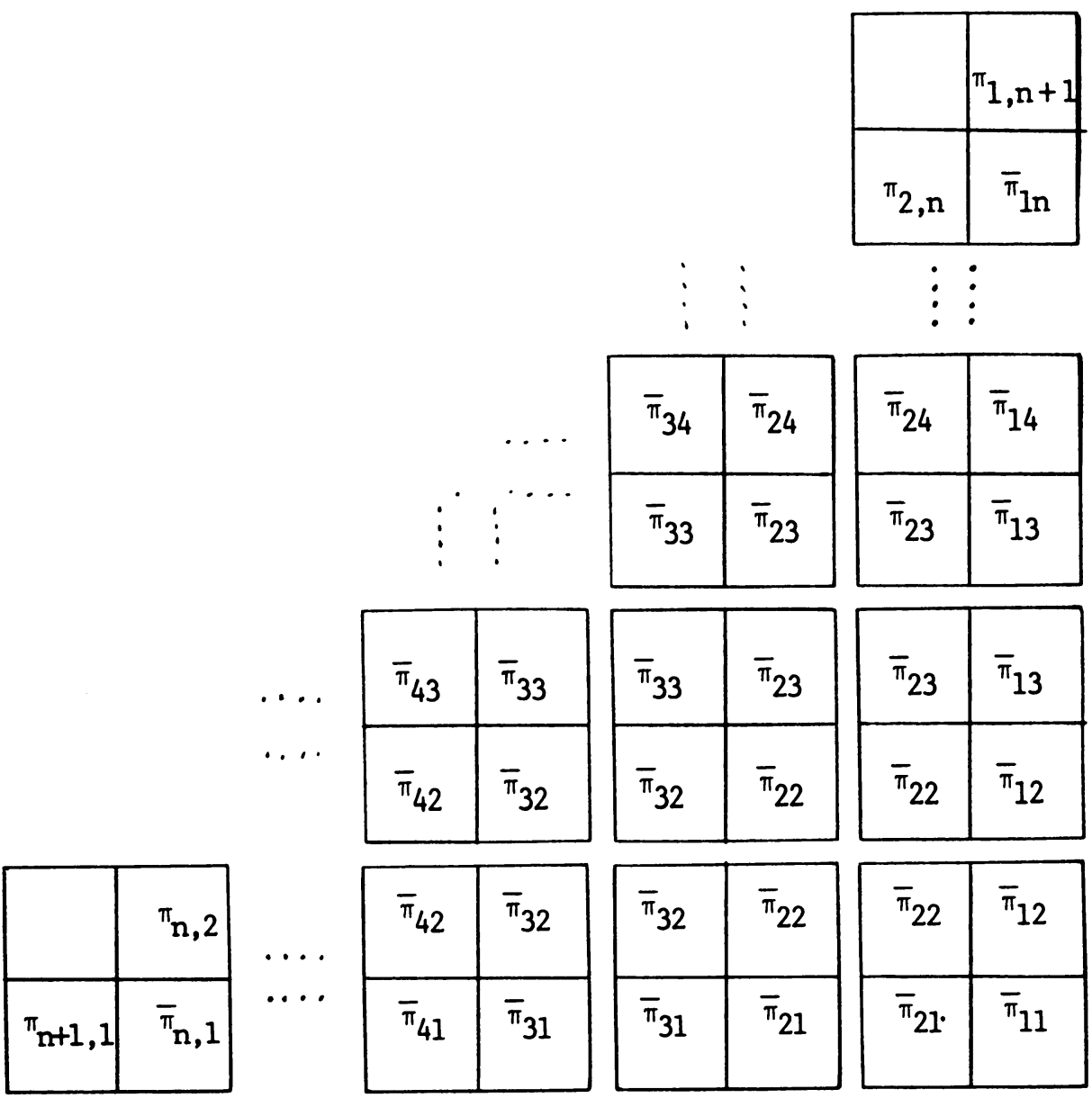

The standard representations $\pi_{i j}$ "along the diagonal" in Table 8 are Schmid embeddings of the discrete series $\pi_{1+i, n+1-i}, 0 \leqslant i \leqslant n$. Consequently, following the conventions of (7.6):

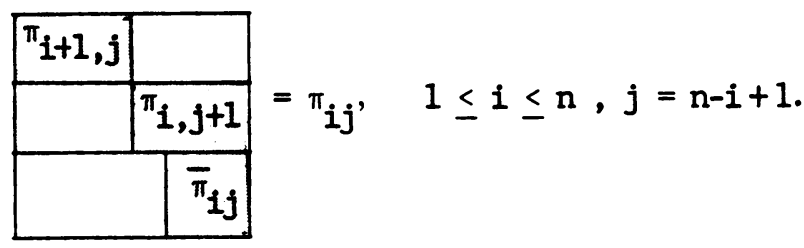


This observation, Table 8 and reasoning as in (7.7), yield

(A.2.4) Theorem. Let $G=\operatorname{SU}(n, 1)(n \geqslant 2)$. Then the UEP-modules are among the representations: $\bar{\pi}_{11}, \bar{\pi}_{12}, \ldots, \bar{\pi}_{1 n} ; \bar{\pi}_{21}, \bar{\pi}_{31}, \ldots, \bar{\pi}_{n 1}$ (nontempered); $\pi_{n+1,1}$ and $\pi_{1, n+1}$ (discrete series).

(A.2.5) Corollary. Let $G=\mathrm{SU}(n, 1)(n \geqslant 2)$. Then the UEP-modules are precisely the regular irreducible constituents of coherent continuations of the holomorphic discrete series. Furthermore, $\mathbf{L}$ is faithful (recall (5.22)), with $B_{1}=\left\{\alpha_{1}\right\} \cup B_{m}$ and $B_{2}=\left\{\alpha_{n}\right\} \cup B_{m}$; notation is as in Table 7 and (2.20).

Proof. By (5.30) we need only verify the first assertion of (A.2.5) for nontempered representations. Theorem (A.2.4) shows there are at most $2 n-1$ distinct nontempered UEP-modules attached to $\chi$. We will show that continuations of holomorphic discrete series give at least $2 n-1$ distinct UEP-modules. In keeping with the notation of $\S 5$, let $\pi(\psi, \gamma)=\pi_{n+1,1}$ and $\pi\left(\psi^{\prime}, \gamma^{\prime}\right)=\pi_{1, n+1}$. As was noted in the proof of (5.35):

Let $\left\{\gamma=\gamma_{0}, \gamma_{1}, \ldots, \gamma_{n}\right\}$ be the $W\left(\mathfrak{g}, \mathfrak{h}^{c}\right)$-orbit of $\gamma$ in the " $\Phi^{c}\left(\mathfrak{h}^{c}\right) \cap \psi$-chamber". Then $\pi\left(\psi, \gamma_{i}\right)$ contains a unique irreducible subquotient $Q_{\psi}\left(\gamma_{i}\right)$ of lowest $\mathfrak{h}^{c}$-weight $\omega_{0} \gamma_{i}+\delta$; recall, $\omega_{0}$ is the opposition involution of $W\left(\mathfrak{f}, \mathfrak{h}^{c}\right)$.

As a corollary to this remark and the proof of (5.15), we pick up $n$ distinct nontempered UEP-modules by continuing $\pi(\psi, \gamma)$. (We have used the fact $G$ has $n+1$ "types" of discrete series.) A similar remark holds for $\pi\left(\psi^{\prime}, \gamma^{\prime}\right)$ : we pick up $n$ distinct nontempered UEP-modules by continuing $\pi\left(\psi^{\prime}, \gamma^{\prime}\right)$. By (5.27) and (3.37) we have $2 n-1$ distinct nontempered UEP-modules accounted for. The first assertion of (A.2.5) has now been established.

The above argument actually shows that each UEP-module is of the form $Q_{\psi}(\gamma)$ or $Q_{\psi^{\prime}}\left(\gamma^{\prime}\right)$. By construction, $Q_{\psi}(\gamma)$ (resp. $Q_{\psi^{\prime}}\left(\gamma^{\prime}\right)$ ) has lowest $\mathfrak{h}^{c}$-weight $\omega_{0} \gamma+\delta$ (resp. $\omega_{0} \gamma^{\prime}+\delta^{\prime}$ ). Further, $\omega_{0} \gamma+\delta$ and $\omega_{0} \gamma^{\prime}+\delta^{\prime}$ are antidominant with respect to $\Phi^{c}\left(\mathfrak{h}^{c}\right) \cap \psi=\Phi^{c}\left(\mathfrak{h}^{c}\right) \cap \psi^{\prime}$. The Cayley transforms $c_{s_{m}}$ and $c_{s_{m}^{\prime}}((5.7))$ show $\left(Q_{\psi}(\gamma)\right)^{\hat{n}}$

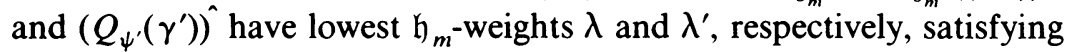

$$
\left(\lambda \mid c_{s_{m}}\left(\Phi^{c}\left(\mathfrak{h}^{c}\right)\right) \cap \Phi^{+}\left(\mathfrak{g}, \mathfrak{h}_{m}\right)\right) \leqslant 0
$$

and

$$
\left(\lambda^{\prime} \mid c_{s_{m}^{\prime}}\left(\Phi^{c}\left(\mathfrak{h}^{c}\right)\right) \cap \Phi^{+}\left(\mathfrak{g}, \mathfrak{h}_{m}\right)\right) \leqslant 0 .
$$

Since $\left(\lambda \mid B_{m}\right) \leqslant 0$ and $\left(\lambda^{\prime} \mid B_{m}\right) \leqslant 0$, we must have (recall $\left.(2.2)\right)\left\langle c_{s_{m}}\left(\Phi^{c}\left(\mathfrak{h}^{c}\right)\right) \cap \Phi^{+}\right.$ $\left.\left(\mathfrak{g}, \mathfrak{h}_{m}\right)\right\rangle=\left\langle B_{m} \cup\left\{\alpha_{i}\right\}\right\rangle, i=1$ or $n$, as in Table 7. A similar statement holds for $c_{s_{m}^{\prime}}$. Q.E.D.

(A.2.6) Lemma. Let $G=\mathrm{SU}(n, 1)$ and $\lambda \in \mathbf{L}$; recall (5.21). There is one and only one UEP-module associated to $\lambda$.

Proof. Assume distinct $V$ and $V_{1}$ are associated to $\lambda$. Then $V$ and $V_{1}$ embed into the same generalized principal series along $P_{m}$. This is impossible for a nontempered parameter by (3.6). Table 8 precludes the possibility of $\lambda$ being a tempered parameter. Q.E.D. 


\section{REFERENCES}

1. A. Borel and N. Wallach, Continuous cohomologv, discrete subgroups, and representations of reductive groups, Ann. of Math. Studies, no. 94, Princeton Univ. Press, Princeton, N.J., 1980.

2. W. Casselman, Jacquet modules for real reductive groups, Proc. Internat. Congr. Math., Helsinki, 1978, pp. 557-563.

3. W. Casselman and D. Miličić, Asvmptotic behavior of matrix coefficients of admissible representations, Duke Math. J. 49 (1982), 869-930.

4. J. Dixmier, Algèbres enveloppantes, Cahiers Sci., vol. 37, Gauthier-Villars, Paris, 1974.

5. Harish-Chandra, Invariant eigendistributions on a semisimple Lie group, Trans. Amer. Math. Soc. 119 (1965), 457-508.

6. __ Discrete series for semisimple Lie groups. II, Acta Math. 116 (1966), 1-111.

7. ___ Representations of semisimple Lie groups. II, Trans. Amer. Math. Soc. 76 (1954), 26-65.

8. H. Hecht, The characters of some representations of Harish-Chandra, Math. Ann. 219 (1976), 213-226.

9. H. Hecht and W. Schmid, A proof of Blattner's conjecture, Invent. Math. 31 (1975), 129-154.

10. Characters, asymptotics and n-homology of Harish-Chandra modules, Acta Math. (to appear).

11. On the asymptotics of Harish-Chandra modules (preprint).

12. S. Helgason, Differential geometry, Lie groups, and symmetric spaces, Academic Press, New York, 1978.

13. A. Knapp and N. Wallach, Szegö kernels associated with discrete series, Invent. Math. 34 (1976), 163-200.

14. A. Knapp and G. Zuckerman, Classifications of irreducible tempered representations of semisimple groups, Ann. of Math. 116 (1982), 389-455.

15. H. Kraljevic and M. W. Baldoni Silva, Composition factors of the principal series representations of the group SP( $n, 1)$, Trans. Amer. Math. Soc. 262 (1980), 447-471.

16. R. Langlands, On the classification of irreducible representations of real algebraic groups, Mimeographed Notes, Institute for Advanced Study, Princeton, N.J., 1973.

17. D. Miličic, Asymptotic behavior of matrix coefficients of the discrete series, Duke Math. J. 44 (1977), 59-88.

18. M. Primc, Representations of semisimple Lie groups with one conjugacy class of Cartan subgroups, Glas. Math. Ser. III 14 (34) (1979), 227-254.

19. W. Schmid, On the characters of the discrete series (the Hermitian symmetric case), Invent. Math. 30 (1975), 47-144.

20. Vanishing theorems for Lie algebra cohomology and the cohomology of discrete subgroups of semisimple Lie groups, Adv. in Math. 41 (1981), 78-113.

21. Two character identities for semisimple Lie groups, Noncommutative Harmonic Analysis, Lecture Notes in Math., vol. 587, Springer-Verlag, Berlin and New York, 1977, pp. 196-225.

22. M. W. Baldoni Silva, The embeddings of the discrete series in the principal series for semisimple Lie groups of real rank one, Trans. Amer. Math. Soc. 261 (1980), 303-368.

23. B. Speh and D. Vogan, Reducibility of generalized principal series representations, Acta Math. 145 (1980), 227-299.

24. E. Thieleker, On the quasi-simple irreducible representations of the Lorentz groups, Trans. Amer. Math. Soc. 179 (1973), 465-505.

25. J. Vargas, A character formula for the discrete series of a semisimple Lie group, Ph.D. Dissertation, Columbia University, New York, 1977.

26. D. Vogan, Gelfand-Kirillow dimension for Harish-Chandra modules, Invent. Math. 48 (1978), 75-98.

27. ___ Irreducible characters of semisimple Lie groups. I, Duke Math. J. 46 (1979), 61-108.

28. Irreducible characters of semisimple Lie groups. II: The Kazhdan-Lusztig conjectures, Duke Math. J. 46 (1979), 805-859.

29. __ Irreducible characters of semisimple Lie groups. III: Proof of the Kazhdan-Lusztig conjectures in the integral case, Invent. Math. 71 (1983), 381-417. 1981. , Representations of real reductive Lie groups, Progress in Mathematics, Birkhauser, Basel,

31. N. Wallach, Square integrable automorphic forms and cohomology of arithmetic quotients of $\operatorname{SU}(p, q)$ (preprint). 
32. Representations of semisimple Lie groups and Lie algebras, Queen's Papers in Pure and Appl. Math. 48 (1977), 154-247.

33. D. Zelobenko, $A$ description of the quasi-simple irreducible representations of the groups $U(n, 1)$ and $\operatorname{SPIN}(n, 1)$, Math. USSR-Izv. 11 (1977), 31-50.

34. G. Zuckerman, Tensor products of finite and infinite dimensional representations of semisimple Lie groups, Ann. of Math. (2) 106 (1977), 295-308.

Department of Mathematics, University of Utah, Salt lake City, Utah 84112

Current address: School of Mathematics, Institute for Advanced Study, Princeton, New Jersey 08540 